Aus dem Institut für Diagnostische und Interventionelle Radiologie (Prof. Dr. med. J. Lotz) im Zentrum Radiologie der Medizinischen Fakultät der Universität Göttingen

\title{
Evaluation eines Echtzeit-Verfahrens zur quantitativen Flussmessung in der kardialen Magnetresonanztomographie
}

\author{
INAUGURAL - DISSERTATION \\ zur Erlangung des Doktorgrades \\ der Medizinischen Fakultät der \\ Georg-August-Universität zu Göttingen
}

vorgelegt von

Johannes Tammo Kowallick

aus

Göttingen

Göttingen 2016 
Dekan:

I. Berichterstatter:

II. Berichterstatter:

III. Berichterstatter/in:
Prof. Dr. rer. nat. H. K. Kroemer

Prof. Dr. med. J. Lotz

Priv.-Doz. Dr. med. C. Jacobshagen

Tag der mündlichen Prüfung: 05.04.2016 


\section{Inhaltsverzeichnis}

\section{Abkürzungsverzeichnis}

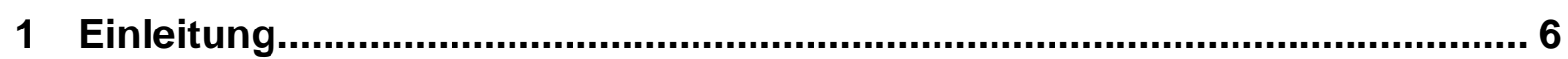

1.1 Quantitative Flussmessung in der kardialen Magnetresonanztomo-

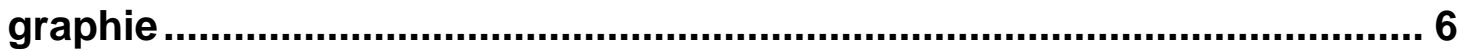

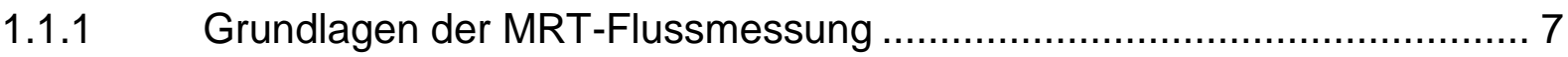

1.1.2 Konventionelle CINE-Flussmessung und Echtzeit-Flussmessung ......... 10

1.1.3 Auswertung von MRT-Flussmessungen ...................................... 12

1.1.4 Anforderungen bei MRT-Flussmessungen: Phasen-Offset-Fehler und andere Fehlerquellen ........................................................ 13

1.2 Vergleich mit der Doppler-Echokardiographie ...................................... 14

1.3 Anwendung der Echtzeit-CMR-Flussmessung mit dem Valsalva-Manöver als physiologischem Stressor ............................................................... 15

1.4 Ziele und Fragestellung ...................................................................... 16

2 Material und Methoden ................................................................................ 17

2.1 Vergleich von Echtzeit-CMR-Flussmessung und CINE-Flussmessung .. 17

2.1.1 In-Vitro-Evaluation der MRT-Flussmessungen zum Auftreten von PhasenOffset-Fehlern ..................................................................... 18

2.1.1.1 Herstellung des Phantoms zur Messung von Phasen-Offset-Fehlern 18

2.1.1.2 Durchführung der Phantommessungen zur Erfassung von PhasenOffset-Fehlern ................................................................. 20

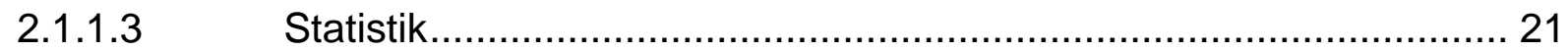

2.1.2 In-Vivo-Evaluation der Echtzeit-CMR-Flussmessung........................ 21

2.1.2.1 Vergleich hämodynamischer Parameter .................................... 24

2.1.2.2 Vergleich der Auswertbarkeit................................................. 25

2.1.2.3 Vergleich mit der Doppler-Echokardiographie ............................ 27

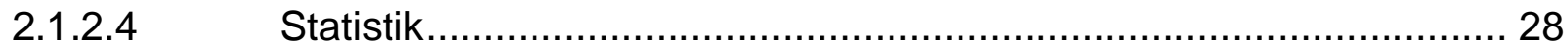

2.2 Anwendung der Echtzeit-CMR-Flussmessung mit dem Valsalva-Manöver als physiologischem Stressor ........................................................... 29

2.2.1 Experimenteller Versuchsaufbau und Versuchsdurchführung................29

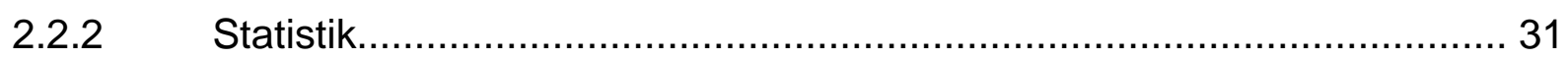




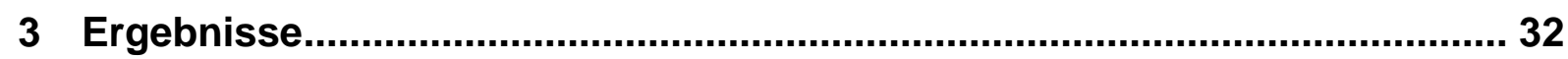

3.1 Vergleich von Echtzeit-CMR-Flussmessung und CINE-Flussmessung.. 32

3.1.1 In-Vitro-Evaluation der MRT-Flussmessungen zum Auftreten von Phasen-

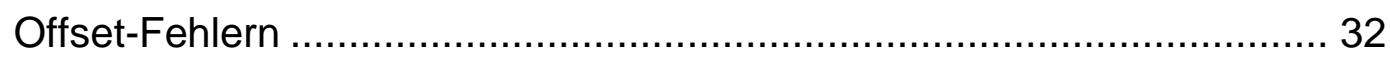

3.1.2 In-Vivo-Evaluation der Echtzeit-CMR-Flussmessung............................ 33

3.1.2.1 Vergleich hämodynamischer Parameter ........................................ 33

3.1.2.2 Vergleich der Auswertbarkeit..................................................... 42

3.1.2.3 Vergleich mit der Doppler-Echokardiographie .............................. 44

3.2 Anwendung der Echtzeit-CMR-Flussmessung mit dem Valsalva-Manöver als physiologischem Stressor .................................................................. 46

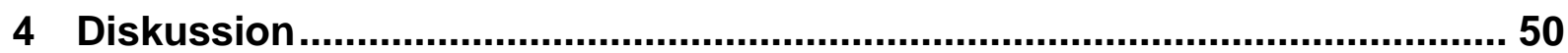

4.1 Vergleich von Echtzeit-CMR-Flussmessung und CINE-Flussmessung.. 50

4.2 Anwendung der Echtzeit-CMR-Flussmessung mit dem Valsalva-Manöver als physiologischem Stressor ................................................................59

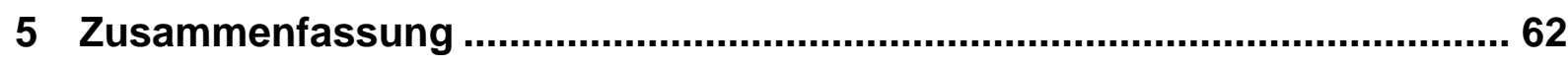

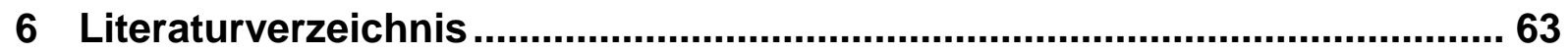

Publikationsliste 


\section{Abkürzungsverzeichnis}

AAo

BMI

Bpm

CINE-BH

CINE-FB

CMR

CW-Doppler

DAo

EKG

FLASH

FOV

GRAPPA

MRT

NLINV

Pixel

SVC

$\mathrm{ROI}$

RT-CMR-Int

RT-CMR-Seq

SD

TE

TR

VENC

Vmax

Voxel
Aorta ascendens

Body Mass Index

beats per minute: Schläge pro Minute

CINE-Phasenkontrast-Flussmessung in Atemstillstand

CINE-Phasenkontrast-Flussmessung in freier Atmung

kardiale Magnetresonanztomographie

Continous Wave-Doppler

Aorta descendens

Elektrokardiogramm

fast low angle shot

field of view: Gesichtsfeld

generalized autocalibrating partially parallel acquisition

Magnetresonanztomographie

iterativ regularisierte nichtlineare Inversion

Bildpunkt

Vena cava superior

region of interest: Für die Auswertung relevanter Bildbereich

kardiale Echtzeit-MRT-Flussmessung mit verschachtelter Flusskodierung

kardiale Echtzeit-MRT-Flussmessung mit sequentieller Flusskodierung

Standardabweichung

echo time: Echozeit

repetition time: Repetitionszeit

encoding velocity: Kodiergeschwindigkeit einer Phasenkontrast-

Flussmessung

Maximalgeschwindigkeit

Volumenpixel 


\section{Einleitung}

\subsection{Quantitative Flussmessung in der kardialen Magnetresonanztomographie}

Die quantitative Flussmessung in der kardialen Magnetresonanztomographie (MRT) basiert auf der Phasenkontrast-Technik (Firmin et al. 1990). Die Ursprünge dieser Technik sind bereits in den 1960er Jahren zu finden, als Erwin Hahn erstmals die Bewegung von Meerwasser durch die Anwesenheit eines äußeren Magnetfeldes darstellten konnte (Hahn 1960). Damit wurden die ersten Ansätze der MRTFlussmessung sogar noch vor der Veröffentlichung der grundlegenden Prinzipien der morphologischen Magnetresonanz(MR)-Bildgebung von Lauterbur und Mansfield gezeigt (Lauterbur 1973; Mansfield and Maudsley 1977). Der Nutzen der damals hoch innovativen Technik wurde schon früh gerade für den Bereich der kardiovaskulären Bildgebung erkannt. Dennoch hat die Translation in die klinische Anwendung fast 30 Jahre gedauert. Die hohe Verfügbarkeit konkurrierender nichtinvasiver Verfahren wie die Doppler-Echokardiographie sowie die Notwendigkeit einer aufwendigen Nachverarbeitung standen der klinischen Verbreitung zunächst im Weg. Mit der Einführung leistungsstarker MR-Geräte und dem Aufkommen einfacher Nachverarbeitungsprogramme fand das Verfahren in den späten 1990er Jahren schließlich einen breiten Einzug in die klinische Nutzung.

Die MRT-Flussmessung gilt heutzutage als theoretisch wie auch klinisch validiert und findet ihren Einsatz vor allem auf dem Gebiet der kongenitalen Herzvitien (Cawley et al. 2009). Mittlerweile gilt sie bei bestimmten Fragestellungen - wie der Quantifizierung der Pulmonalklappeninsuffizienz - bei der Entscheidung zur Intervention als zuverlässig einsetzbar und anderen Verfahren sogar überlegen (Achenbach et al. 2012). Trotzdem beinhaltet die zugrunde liegende Technik in vielen Situationen bedeutsame Limitationen für die klinische Anwendung. Dies hat in der Vergangenheit zur Entwicklung noch schnellerer Techniken - den so genannten Echtzeit-Verfahren - geführt (Debatin et al. 1995 a; Korperich et al. 2004). Aufgrund der schlechten Ortsauflösung und der hohen Artefaktanfälligkeit konnten sich diese Verfahren bisher allerdings noch nicht in der klinischen Routine etablieren. 


\subsubsection{Grundlagen der MRT-Flussmessung}

Die MRT basiert auf dem Verhalten magnetischer Momente (Spins) in einem äußerlich angelegten Magnetfeld. Im menschlichen Körper stellen die Kerne der Wasserstoffprotonen den Großteil der Spins dar. In Abhängigkeit des Körpergewebes zeigen die Spins verschiedene Verhaltensmuster im magnetischen Feld. Diese Variationen machen eine Differenzierung der Gewebe in der morphologischen wie auch in der
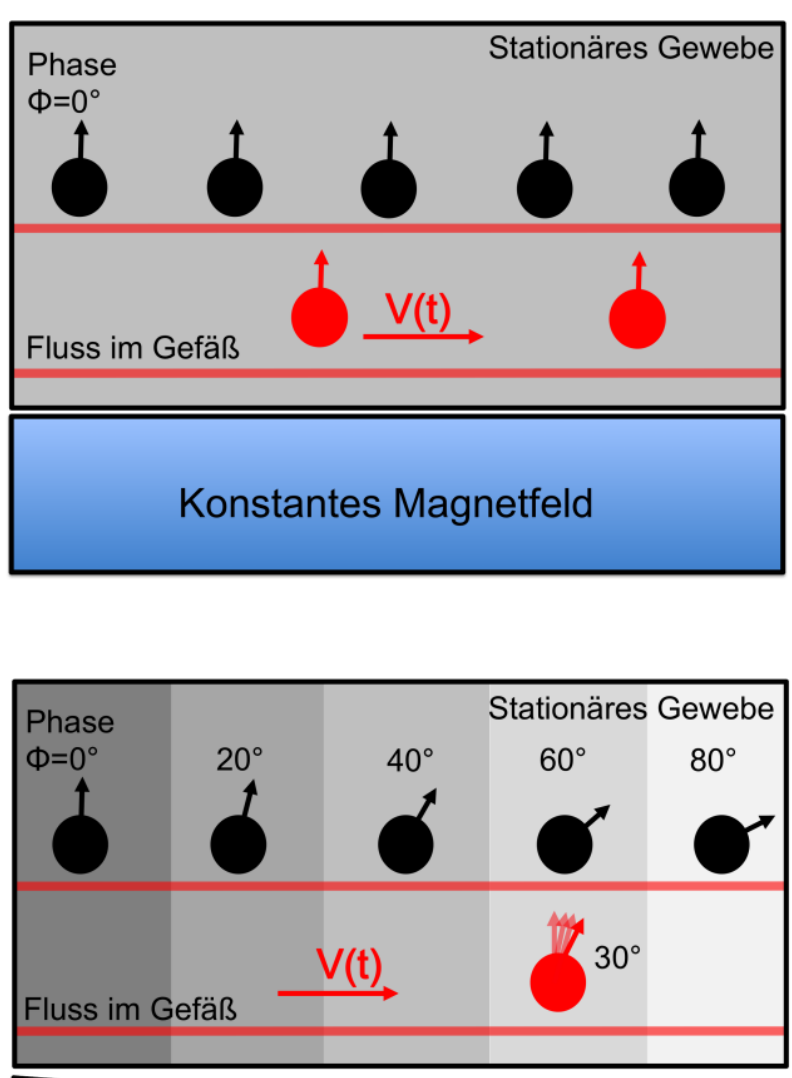

Linearer Magnetfeldgradient

Abbildung 1: Oben: In einem konstanten Magnetfeld weisen Spins in stationärem Gewebe und in bewegtem Gewebe (Fluss im Gefäß) die gleiche Phase $(\Phi)$ auf.

Unten: Spins, die sich im Blutgefäß entlang eines linearen Magnetfeldgradienten bewegen, nehmen anteilig eine Phasenänderung auf. Die Phase unterscheidet sich dadurch von der Phase stationärer Spins in gleicher Schichtposition (modifiziert nach Lotz et al. 2002, S. 652). funktionellen MR-Bildgebung möglich. Im Zentrum der MRT-PhasenkontrastFlussmessung steht die Relation bewegter Spins (z. B. innerhalb eines Blutgefäßes) zu stationären Spins (z. B. in der Gefäßwand).

Spins, die sich in einem äußerlich angelegten Magnetfeld befinden, erfahren eine Rotationsbewegung entlang der magnetischen Feldlinien - die so genannte Präzessionsbewegung. Die Geschwindigkeit dieser Bewegung wird mit Hilfe der Präzessionsfrequenz beschrieben, die auch als Resonanzfrequenz bezeichnet werden kann. Die Resonanzfrequenz ist abhängig von der Stärke des äußerlichen Magnetfeldes. In einem konstanten Magnetfeld besitzen alle Spins - sowohl stationäre als auch bewegte Spins - die gleiche Resonanzfrequenz (Abbildung 1). Durch eine Erhöhung des äußerlichen Magnetfeldes erhöht sich ebenfalls die Resonanzfrequenz der Spins. Durch eine Verringerung des Magnetfeldes kommt es entsprechend zu einer Er- 
niedrigung der Resonanzfrequenz. Feine Variationen des äußerlichen Magnetfeldes können in der MR-Bildgebung durch die Applikation von Magnetfeldgradienten erreicht werden. Durch die geringen Unterschiede des äußeren magnetischen Feldes weisen die Spins in stationärem Gewebe zueinander verschiedene Resonanzfrequenzen auf. Man spricht in diesem Zusammenhang auch von einer veränderten Phase zueinander. Spins, die sich in den Blutgefäßen entlang eines Magnetfeldgradienten bewegen, erfahren nur anteilig eine Änderung der Resonanzfrequenz. Die Phase bewegter Spins unterscheidet sich entsprechend in gleicher Position von der Phase angrenzender stationärer Spins, man spricht von einer Phasendifferenz (Abbildung 1). Unter der Verwendung linearer Magnetfeldgradienten konnte gezeigt werden, dass die Phasendifferenz proportional zur Geschwindigkeit bewegter Spins ist (Moran et al. 1985). Streng genommen gilt dies nur unter der Voraussetzung, dass während der Messung ein konstant laminarer Blutfluss im Gefäß herrscht.

Die Phasendifferenz bewegter Spins relativ gesehen zu stationären Spins kann nur gemessen werden, wenn die Phasenänderung stationärer Spins kompensiert wird. Aus diesem Grund wird der zuerst geschaltete Magnetfeldgradient anschließend mit umgekehrter Polarität wiederholt (Abbildung 2). Durch die Applizierung eines solchen bipolaren Magnetfeldgradienten, der dann auch als flusskodierender Magnet-

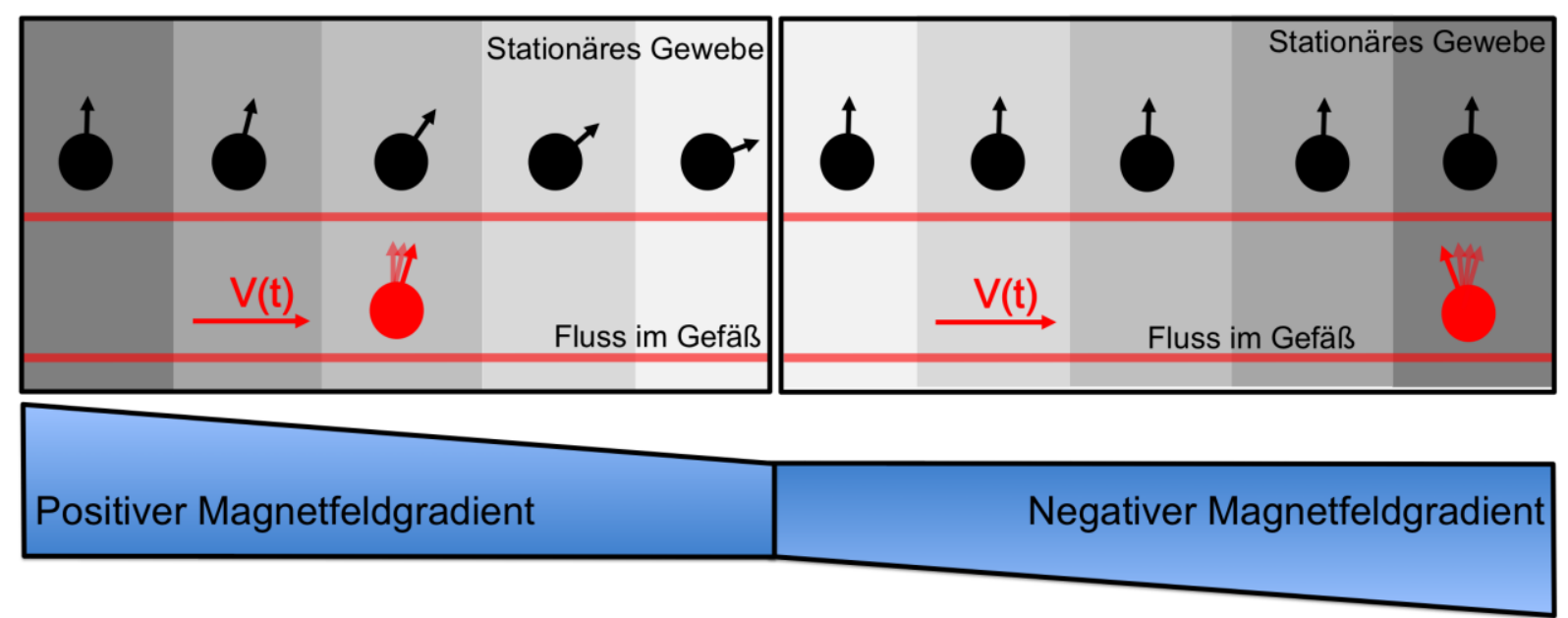

Abbildung 2: Grundlegendes Prinzip der Phasenkontrast-Technik. Die Anlage eines bipolaren Magnetfeldgradienten führt zur Egalisierung der Phasenunterschiede im stationären Gewebe. Relativ dazu erfahren sich bewegende Spins in dem Gefäß nach Schaltung des zweiten Gradienten (negativer Gradient) eine messbare Phasendifferenz (modifiziert nach Lotz et al. 2002, S. 653). 
feldgradient bezeichnet werden kann, werden die zuvor langsameren Spins (niedrige Resonanzfrequenz) beschleunigt, die zuvor schnelleren Spins (hohe Resonanzfrequenz) wieder abgebremst. Spins im stationären Gewebe weisen nach der Schaltung des bipolaren flusskodierenden Gradienten anschließend wieder alle die gleiche Phase auf. Im Gegensatz dazu erreicht die Phase bewegter Spins nicht die gleiche Phase wie angrenzende stationäre Spins, sodass eine Phasendifferenz bestehen bleibt. Üblicherweise wird die gleiche Messung zweimal direkt hintereinander durchgeführt. Bei der zweiten Messung wird der bipolare Magnetfeldgradient leicht modifiziert appliziert. Anschließend werden beide Messungen voneinander subtrahiert. Durch dieses Vorgehen werden alle anderen Einflüsse neutralisiert, die während der Datenakquisition zusätzliche Phasendifferenzen hervorgerufen haben können (Spritzer et al. 1990).

Die Phasendifferenz ist eine gerichtete Größe und wird in Grad angegeben. Sie kann nur die auf einen Kreisbogen begrenzten Werte von $-180^{\circ}$ bis $+180^{\circ}$ annehmen. Darum muss der Untersucher vor der Phasenkontrast-Flussmessung einen Geschwindigkeitsbereich bestimmen. Durch die Definition der so genannten Kodiergeschwindigkeit (VENC) werden die flusskodierenden Magnetfeldgradienten so eingestellt, dass die VENC gerade einer Phasendifferenz von $+/-180^{\circ}$ entspricht. Die VENC ist eine Geschwindigkeit mit der Einheit $\mathrm{cm} \mathrm{s}^{-1}$ und sollte vom Untersucher entsprechend so gewählt werden, dass sie gerade über der maximal zu erwartenden Fluss-

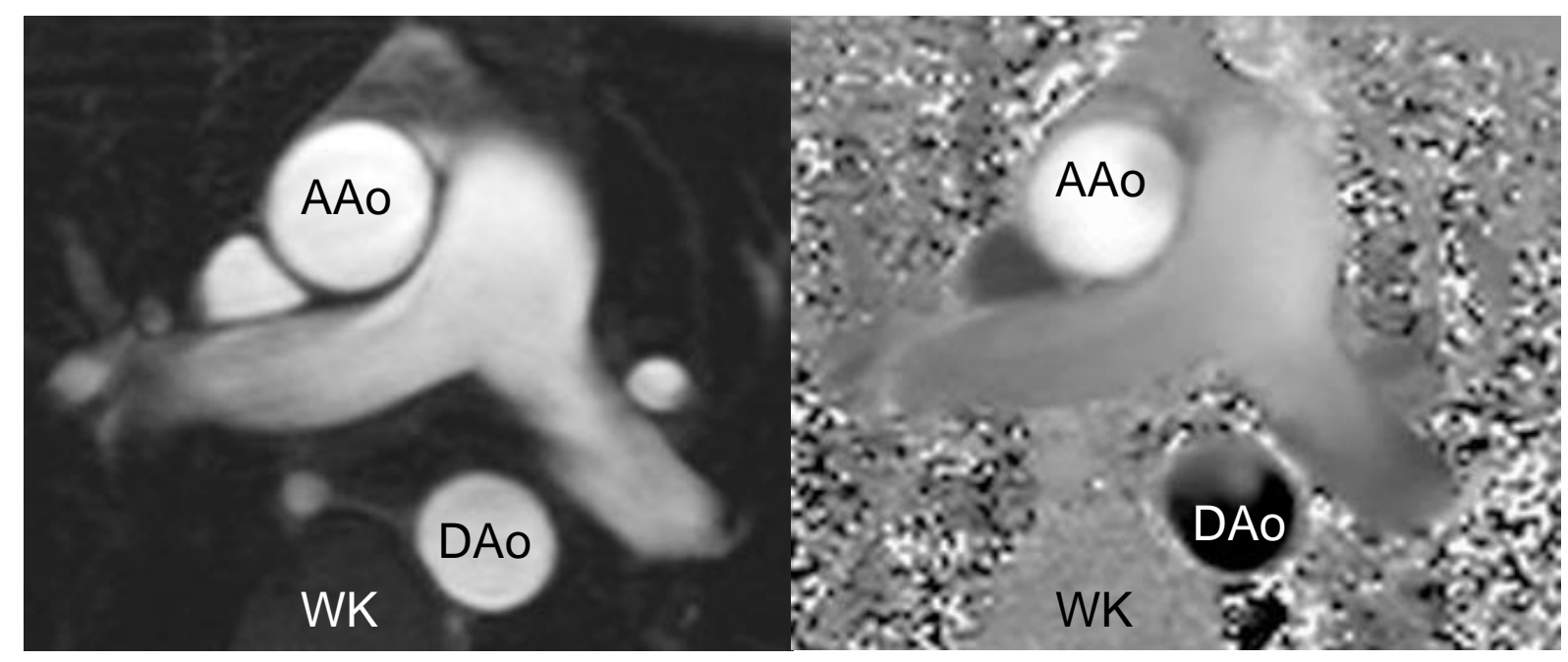

Abbildung 3: Magnitudenbild (links) und Phasenbild (rechts) einer PhasenkontrastFlussmessung in axialer Schnittführung. Aorta ascendens (AAo), Aorta descendens (DAo), Wirbelkörper (WK). 
geschwindigkeit in dem zu untersuchenden Gefäß liegt. Die Verrechnung der Phaseninformation erfolgt bei der Rekonstruktion. Die gemessene Phasendifferenz (Wert zwischen +/-180 ) wird dabei in eine Geschwindigkeit der Größe cm s${ }^{-1}$ umgerechnet (O'Donnell 1985; Pelc et al. 1991). Die Rekonstruktion ergibt typischerweise zwei Datensätze. Zum einen werden so genannte Magnitudenbilder generiert, die zur anatomischen Orientierung dienen, zum anderen werden Phasenbilder rekonstruiert, in denen die eigentlichen Flussinformationen enthalten sind (Abbildung 3). Für jedes Voxel wird die errechnete Geschwindigkeit als Graustufe kodiert dargestellt. Neben der Geschwindigkeitsinformation enthält jedes Voxel im Phasenbild zusätzlich eine Richtungsinformation. Sehr helle Graustufen kodieren dabei für schnelle Flussgeschwindigkeiten in Betrachtungsrichtung, sehr dunkle Graustufen kodieren entsprechend für schnelle Flussgeschwindigkeiten in die entgegengesetzte Richtung. Diese Eigenschaften lassen sich bei Betrachtung der Aorta ascendens (helle Graustufen) und der Aorta descendens (dunkle Graustufen) in Abbildung 3 am besten nachvollziehen. Stationäres Gewebe weist eine mittlere Graustufe auf, wie sie im Bereich des Wirbelkörpers erkennbar ist.

\subsubsection{Konventionelle CINE-Flussmessung und Echtzeit-Flussmessung}

Als Echtzeit-MRT werden MR-Verfahren bezeichnet, bei denen die notwendigen Daten für einen Herzzyklus innerhalb der Zeit eines einzigen Herzschlags vollständig aufgenommen werden können. Alle derzeit im klinischen Altag gebräuchlichen Phasenkontrast-Flussmessungen (CINE-Flussmessungen) entsprechen dieser Anforderung bisher nicht. Um eine adäquate zeitliche Auflösung zu erreichen, sind sie auf die Datenakquisition multipler Herzzyklen angewiesen. Die Information mehrerer Herzzyklen wird interpoliert und in dem Flussprofil eines "synthetischen“ Herzzyklus angegeben. Dazu ist es notwendig, die Datenaufnahme mit dem Herzzyklus zu synchronisieren. Für CINE-Flussmessungen wird typischerweise ein retrospektives EKG-Gating verwendet. Das bedeutet, dass die Datenaufnahme kontinuierlich über mehrere Herzzyklen vorgenommen wird und im Nachhinein mit der R-Zacke eines mitregistrierten EKG synchronisiert wird. Die Interpolation mehrerer Herzzyklen zu einem „synthetischen“ Herzschlag geht unweigerlich mit Limitationen für die klinische Anwendung einher (Ferreira et al. 2013). Es existieren Varianten der CINEFlussmessungen, die in freier Atmung oder in Atemstillstand durchgeführt werden 
können. Beide Möglichkeiten haben Vor- und Nachteile für die klinische Anwendung. Die Messung in Atemstillstand ist einerseits schneller, und Atemartefakte werden vermieden. Andererseits ist die zeitliche Auflösung geringer und die Technik setzt voraus, dass der Patient in der Lage ist, den Atem bis zu 15 Sekunden anzuhalten. Die Messung in freier Atmung dauert einerseits deutlich länger und Atemartefakte können die Qualität der Flussmessung beeinträchtigen. Andererseits ist die zeitliche Auflösung höher und eine Eignung für Patienten, die den Atem nicht über einen längeren Zeitraum anhalten können, ist gegeben.

Die für den klinischen Einsatz bedeutsamen Limitationen der CINE-Flussmessung haben zur Entwicklung einer Reihe von Echtzeit-Flussmessungen geführt (Hansen et al. 2012; Keenan und Pennell 2007; Macgowan et al. 2005; Steeden et al. 2010). Die Schwierigkeit bei der Konzipierung von Echtzeit-Verfahren liegt im Allgemeinen darin, trotz der extrem erhöhten zeitlichen Auflösung die räumliche Auflösung und die Bildqualität aufrechtzuerhalten. Keines der bisher vorgestellten Echtzeit-Verfahren konnte diesen Anforderungen gerecht werden und sich fest in der klinischen Bildgebung etablieren.

Das in der vorliegenden Arbeit verwendete Verfahren geht auf neue Entwicklungen der biomedizinischen NMR Forschungs GmbH am Max Planck Institut für biophysikalische Chemie in Göttingen zurück, die erstmals MRT-Aufnahmen in Echtzeit mit überraschend hoher räumlicher Auflösung und Bildqualität erlauben (Niebergall et al. 2013; Uecker et al. 2008; Uecker et al. 2010 a; Zhang et al. 2010 a; Zhang et al. 2012). Dieses Verfahren eröffnet eine Vielzahl von völlig neuen Anwendungen in der medizinischen Diagnostik, die von Gelenkbewegungen bis zu Schluck- und Sprechbewegungen reichen und sich insbesondere für Untersuchungen des Herzens und der großen Gefäße eignen (Uecker et al. 2010 b; Zhang et al. 2010 b). Durch eine systematische Weiterentwicklung lässt sich diese Technik auch für quantitative Phasenkontrast-Flussmessungen nutzen. In einer 2012 erschienenen Publikation konnte die grundlegende Validierung dieses Ansatzes gezeigt werden. Dabei wurden die Ergebnisse der Echtzeit-Flussmessung am Flussphantom validiert und bei wenigen Probanden als Machbarkeitsstudie erfolgreich durchgeführt (Joseph et al. 2012). Die Technik ist in der Lage, Flussprofile sukzessiver Herzzyklen mit einer realen zeitlichen Auflösung von $40 \mathrm{~ms}$ aufzunehmen. Eine EKG-Synchronisation oder Atemkompensation ist bei der Verwendung nicht erforderlich. 


\subsubsection{Auswertung von MRT-Flussmessungen}

Alleine die Magnituden- und Phasenbilder einer MRT-Flussmessung ergeben noch keine hämodynamischen Parameter (z. B. Flussvolumen, Maximalgeschwindigkeit)
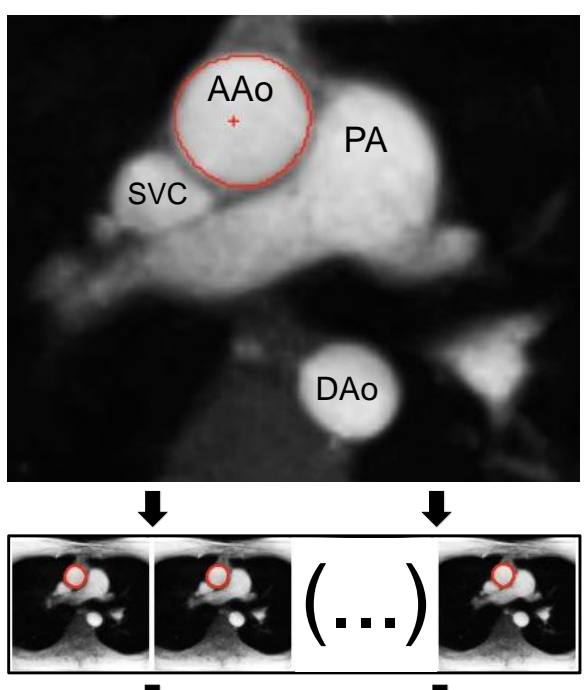

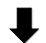

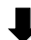

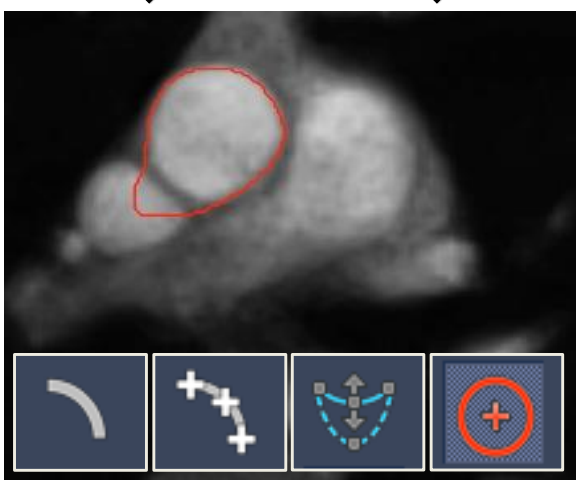

Abbildung 4: Auswertung einer MRT-Flussmessung in der Aorta ascendens (AAo). Definition einer initialen ROI im Magnitudenbild (oben). Automatische Übertragung der Kontur auf alle Phasen (Mitte). Korrekturbedürftige Kontur; manuelle Korrektur-Tools stehen zur Verfügung (unten). Truncus pulmonalis (PA), Aorta descendens (DAo), Vena cava superior (SVC). der unterschiedlichen Zielgefäße. Daher ist es notwendig, die rekonstruierten Bilder in einem geeigneten Auswertungsprogramm zu analysieren. Es existieren Software-Pakete unterschiedlicher Hersteller, deren Grundprinzip jedoch gleich ist (Abbildung 4). Alle Programme unterstützen das Einzeichnen von Bildbereichen (ROI) um das zu analysierende Gefäß. Die Einzeichnung einer initialen ROI wird typischerweise im Magnitudenbild vorgenommen. Ein automatischer Segmentationsalgorithmus ist anschließend in der Lage, die initiale $\mathrm{ROI}$ auf allen Magnitudenbildern zu ergänzen und auf die Phasenbilder zu übertragen. Der dahinterliegende Algorithmus orientiert sich an der unterschiedlichen Kontrastierung von Gefäßlumen und angrenzendem Gewebe. Die Kontur sollte im Idealfall kongruent zur Gefäßintima liegen. Der automatische Algorithmus erkennt die gewünschte $\mathrm{ROI}$ in einigen Phasen allerdings nicht optimal. Deshalb ist es nötig, nach der automatischen Übertragung die $\mathrm{ROI}$ aller Phasen visuell zu überprüfen und, falls erforderlich, manuell zu korrigieren. Üblicherweise stehen unterschiedliche Werkzeuge für die manuelle Konturkorrektur zur Verfügung. Die hämodynamischen Parameter werden aus den Graustufen der ROI-umfassten Pixel ermittelt und können in einem Auswertungs-Report angezeigt werden.

Die Anwendung von Echtzeit-MRT-Flussmessungen stellt einige Anforderungen an die Auswertungssoftware, die bei konventionellen CINE-Flussmessungen bisher keine Rolle spielten. Beispielsweise ergibt eine Echtzeit-Flussmessung (zeitliche Auflö- 
sung: $40 \mathrm{~ms}$ ) bei einer 10-sekündlichen Anwendung mehr als die 10-fache Datenmenge (375 Phasen) einer konventionellen CINE-Flussmessung, bei der üblicherweise pro Messung nur 25-30 Phasen rekonstruiert werden. Der zeitliche Aufwand für die manuelle Konturkorrektur kann sich entsprechend aufwändiger gestalten. Des Weiteren kann die Erhöhung der zeitlichen Auflösung mit Einbußen der Bildqualität einhergehen. Dies könnte der Abgrenzbarkeit des Zielgefäßes von umliegenden Strukturen schaden und somit den manuellen Korrekturaufwand erhöhen. Ein geeignetes automatisches Auswertungsprogramm ist deshalb gerade für die Analyse von Echtzeit-Daten unumgänglich. Die Zuverlässigkeit und die klinisch akzeptable Handhabung der Auswertbarkeit soll im Rahmen der vorliegenden Arbeit überprüft werden.

\subsubsection{Anforderungen bei MRT-Flussmessungen: Phasen-Offset-Fehler und an- dere Fehlerquellen}

Bei der Anwendung von MRT-Flussmessungen lassen sich grundsätzlich die vom Untersucher beeinflussbaren sowie die unbeeinflussbaren Fehlerquellen unterscheiden.

Zu den vom Untersucher beeinflussbaren Fehlerquellen zählt die Wahl einer zu geringen bzw. zu hohen VENC bei der Planung einer Flussmessung, was zu Einfaltungsartefakten (Aliasing) bzw. einem erhöhten Rauschen führen kann. Die Schnittebene sollte im Idealfall orthogonal zur Flussrichtung liegen. Abweichungen, die über ein $\mathrm{Maß}$ von $15^{\circ}$ von der Orthogonalen hinausgehen, können ebenfalls zu Abweichungen der Flussparameter führen (Tang et al. 1993). Nicht zuletzt können falsch eingezeichnete $\mathrm{ROI}$ bei der Auswertung zu signifikanten Flussabweichungen führen. Alle genannten Einflussgrößen können durch den erfahrenen Untersucher vermieden bzw. durch eine wiederholende Messung oder Auswertung mit angepassten Einflussgrößen relativ einfach korrigiert werden. Ihr Einfluss auf die Güte von MRTFlussmessungen wurde vielfach untersucht (Lotz et al. 2002).

Daneben existieren technische Einflussgrößen, die durch den Untersucher nicht direkt zu beeinflussen sind. Die Theorie der Phasenkontrast-Flussmessung geht von einem kontinuierlichen, laminaren Fluss in dem zu messenden Gefäß aus. Beschleu- 
nigungen oder Turbulenzen, wie sie bei Klappenstenosen oder -insuffizienzen auftreten können, haben deshalb das Potenzial Fehler zu induzieren (Petersson et al. 2010). Eine weitere nichtbeeinflussbare Fehlerquelle stellen Phasen-Offset-Fehler dar. Dies sind systematische Verschiebungen der Geschwindigkeitswerte, die sowohl in stationärem als auch bewegtem Gewebe auftreten können. Je nach Ausmaß können sie zu erheblichen Abweichungen der gemessen Flussparameter führen (Holland et al. 2010). Das Ausmaß hängt dabei in erster Linie von lokalen magnetischen Feldinhomogenitäten und Gradientenfehlern ab (Lotz et al. 2002). Dazu können die Lage des Zielgefäßes relativ zum magnetischen Isozentrum, die Angulierung der Messebene, aber auch die VENC Einfluss auf das Auftreten haben (Chernobelsky et al. 2007). Die Schwierigkeit bei der Messung von Phasen-Offset-Fehlern liegt darin, dass sie sowohl homogen als auch heterogen in der Messebene auftreten können. Sie lassen sich deshalb nur mit Hilfe eines statischen Phantoms zuverlässig bestimmen, an dem die Flussmessung - nach einer Patientenmessung in gleicher Position, wie das zuvor untersuchte Gefäß - wiederholt wird. Im Rahmen der vorliegenden Arbeit soll das Auftreten von Phasen-Offset-Fehlern in der neu entwickelten EchtzeitFlussmessung mit konventionellen CINE-Flussmessungen verglichen werden.

Eine der größten Anforderungen bei allen quantitativen Flussbestimmungen in vivo ist das Fehlen eines allgemein gültigen Referenzstandards (Lotz et al. 2002). Die Angaben über die Güte und die Genauigkeit verschiedener Techniken (MRT Phasenkontrast-Flussmessung, MRT Volumetrie, invasive Thermodilutionsverfahren, Doppler-Echokardiographie) sind Näherungswerte, die in der Regel auf Phantommessungen basieren (Kimme-Smith et al. 1990; Siegel et al. 1996). Der tatsächliche Fehler der in der Arbeit verwendeten MRT-Flussmessungen ist deshalb nur schwer zu ermitteln. Es bleibt die Möglichkeit, die Ergebnisse der Echtzeit-Flussmessung mit anderen etablierten Methoden zu vergleichen.

\subsection{Vergleich mit der Doppler-Echokardiographie}

Im klinischen Kontext ist vor allem der Vergleich der MRT-Flussmessungen mit der Doppler-Echokardiographie relevant. Die maximale Flussgeschwindigkeit entlang einer Stenose wird als Ausdruck des Ausmaßes einer Verengung verwendet. Bei einigen klinischen Fragestellungen basiert die Entscheidung zur Intervention alleine 
auf der nichtinvasiv ermittelten (Doppler-Echokardiographie oder MRTFlussmessung) maximalen Flussgeschwindigkeit (Warnes et al. 2008). Aus diesem Grund steht der Vergleich der Maximalgeschwindigkeiten zwischen EchtzeitFlussmessung, CINE-Flussmessung und Doppler-Echokardiographie im Vordergrund.

Frühere In-Vivo- und In-Vitro-Studien haben gezeigt, dass konventionelle CINEFlussmessungen tendenziell dazu neigen, die maximale Flussgeschwindigkeit im Vergleich zur Doppler-Echokardiographie zu unterschätzen (Siegel et al. 1996). Dies wird mit der schlechteren zeitlichen Auflösung der CINE-Flussmessungen im Vergleich zur Echokardiographie begründet. Im Rahmen der Studie soll überprüft werden, ob die Erhöhung der zeitlichen Auflösung der Echtzeit-Flussmessung dazu führt, dass höhere Maximalgeschwindigkeiten als in den CINE-Flussmessungen gemessen werden und eher denen der Doppler-Echokardiographie entsprechen.

\subsection{Anwendung der Echtzeit-CMR-Flussmessung mit dem Valsalva-Manöver als physiologischem Stressor}

Die physiologischen Variationen des Blutfluss während des Valsalva-Manövers (erhöhter intrathorakaler Druck) wurden historisch mit Hilfe invasiver Verfahren ermittelt (Brooker et al. 1974; Greenfield et al. 1967). Es konnte gezeigt werden, dass eine abnorme Antwort des Blutflusses auf Variationen des intrathorakalen Drucks prognostische Aussagen bei der Herzinsuffizienz haben können (Felker et al. 2006; Lalande et al. 2012; Parisi et al. 1976). Eine zuverlässige und reproduzierbare nichtinvasive Darstellung der hämodynamischen Reaktion auf das Valsalva-Manöver, z. B. mit Hilfe der Doppler-Echokardiographie, gestaltet sich aufgrund der Abhängigkeit anatomisch definierter Schallfenster schwierig (Gindea et al. 1990). Die technischen Eigenschaften der konventionellen CINE-Flussmessungen basieren auf der Interpolation multipler Herzzyklen, die schließlich in einem „synthetischen" Flussprofil angezeigt werden. Physiologische Änderungen des Blutflusses, die während der Datenakquisition auftreten, unterliegen der Mittelung und sind bei der Auswertung nicht erkennbar (vgl. 1.1.2). 
Die Echtzeit-Flussmessung erlaubt dagegen hämodynamische Parameter direkt aufeinanderfolgender Herzzyklen miteinander zu vergleichen. Diese Möglichkeit soll im Rahmen der Studie genutzt werden, um die physiologische Flussantwort des Valsalva-Manövers (erhöhter intrathorakeler Druck) bei herzgesunden Probanden darzustellen. Die freie Wahl der Schnittebene als weiterer Vorteil der MRT soll in diesem Zusammenhang ausgenutzt werden, um die physiologischen Flussvariationen simultan in zwei Gefäßen (Vena cava superior, Aorta ascendens), nicht-invasiv und zuverlässig in Echtzeit zu analysieren.

\subsection{Ziele und Fragestellung}

Ziel der vorliegenden Arbeit ist die Evaluation des neu entwickelten EchtzeitVerfahrens zur quantitativen Flussmessung in der kardialen MRT. Im Einzelnen sind folgende Fragen zu klären:

1. Bestimmung des Ausmaßes an Phasen-Offset-Fehlern in der EchtzeitFlussmessung. Als Referenz dienen konventionelle CINE-Flussmessungen.

2. Zuverlässigkeit der Echtzeit-Flussmessung bei der Erfassung hämodynamischer Parameter (Flussvolumen, Maximalgeschwindigkeit). Als Referenz dienen konventionelle CINE-Flussmessungen.

3. Eignung der Echtzeit-Flussmessung für die Analyse in einer marktüblichen Auswertungssoftware. Als Referenz dienen konventionelle CINE-Flussmessungen.

4. Exaktere Bestimmung der Maximalgeschwindigkeit in den EchtzeitFlussmessungen als in den konventionellen CINE-Flussmessungen. Als Referenz dient die Doppler-Echokardiographie.

5. Eignung der Echtzeit-Flussmessung zur Erfassung physiologischer Flussvariationen während des Valsalva-Manövers (erhöhter intrathorakaler Druck). 


\section{Material und Methoden}

Im Folgenden sollen die einzelnen Arbeitsschritte zur Evaluation des neu entwickelten Echtzeit-MRT-Verfahrens zur quantitativen Flussmessung beschrieben werden (Abbildung 5).

\section{Evaluation der Echtzeit-CMR-Flussmessung}

\section{In-Vitro-Evaluation}

Flussmessung am statischen Phantom zur Quantifizierung von Phasen-Offset-Fehlern

\section{In-Vivo-Evaluation}

Echtzeit-CMR-Flussmessung vs. konventionelle CINE-Flussmessung

$\longrightarrow$ Erfassung hämodynamischer Parameter

$\longrightarrow$ Analysen zur Auswertbarkeit der MRT-Flussmessungen

$\longrightarrow$ Vergleich der Maximalgeschwindigkeit mit der Doppler-Echokardiographie

\section{Anwendung der Echtzeit-CMR-Flussmessung}

Erfassung hämodynamischer Variationen unter dem Valsalva-Manöver

Abbildung 5: Übersicht zur Evaluation der Echtzeit-Flussmessung

\subsection{Vergleich von Echtzeit-CMR-Flussmessung und CINE-Flussmessung}

Das Echtzeit-Verfahren (Echtzeit-CMR-Flussmessung) wurde mit klinisch etablierten und validierten Referenzmethoden (CINE-Phasenkontrast-Flussmessungen) verglichen. Zur Erfassung von Phasen-Offset-Artefakten wurden die zu vergleichenden MRT-Flussmessungen in vitro an einem statischen Phantom durchgeführt. Außer- 
dem wurden die verschiedenen Verfahren in vivo bei einer Probandengruppe durchgeführt, um die Echtzeit-Flussmessung in Bezug auf das Erfassen hämodynamischer Parameter, die Auswertbarkeit der erhobenen Datensätze und den Vergleich mit der Doppler-Echokardiographie zu evaluieren.

\subsubsection{In-Vitro-Evaluation der MRT-Flussmessungen zum Auftreten von Pha- sen-Offset-Fehlern}

Eine zuverlässige Evaluation von Phasen-Offset-Artefakten ist lediglich durch die Messung eines statischen Phantoms möglich (Caprihan et al. 1990). Aus diesem Grund wurde nach einem Rezept von Blechinger et al. unter Laborbedingungen ein statischer Gelatine-Agarose-Phantomkörper hergestellt (Blechinger et al. 1988).

\subsubsection{Herstellung des Phantoms zur Messung von Phasen-Offset-Fehlern}

Für das Phantom wurde ein Behälter mit einem Fassungsvermögen von 8 Litern gewählt, sodass dieser von Umfang und Volumen ungefähr dem Thorax eines Probanden entsprach. Tabelle 1 listet alle Komponenten auf, die für die Herstellung von 8 Litern Phantommasse benötigt wurden.

Tabelle 1: Material zur Herstellung der Phantommasse.

\begin{tabular}{ll}
\hline Produkt & Menge \\
\hline Glycerin & $2880 \mathrm{ml}$ \\
N-Propylalkohol & $796 \mathrm{ml}$ \\
destilliertes Wasser & $6912 \mathrm{ml}$ \\
Trockenagar & $252 \mathrm{~g}$ \\
Gelatine & $928 \mathrm{~g}$ \\
Vaseline & ca. $1000 \mathrm{~g}$ \\
p-Methylbenzoesäure (p-Toluic acid) & $6 \mathrm{~g}$ \\
37\%-Formaldehydlösung & $32 \mathrm{~g}$ \\
Paraffin-Platelets & $600 \mathrm{~g}$ \\
\hline
\end{tabular}


Die einzelnen Herstellungsschritte des statischen Phantomkörpers beinhalteten:

\section{Vorbereitung des Gelatine-Gels}

In einem Behälter mit mindestens 8 Litern Fassungsvermögen wurden $6 \mathrm{~g}$ p-Methylbenzoesäure zusammen mit $332 \mathrm{ml} \mathrm{N}$-Propylalkohol in einem Wasserbad von 90 $100{ }^{\circ} \mathrm{C}$ auf $90^{\circ} \mathrm{C}$ erhitzt. Dazu wurden $1200 \mathrm{ml}$ Glycerol und $2468 \mathrm{ml}$ destilliertes Wasser (Zimmertemperatur) gemischt.

\section{Vorbereitung des Agars}

In einem zweiten Behälter mit mindestens 8 Litern Fassungsvermögen wurden 464 $\mathrm{ml} \mathrm{N-Propylalkohol} \mathrm{mit} 1680 \mathrm{ml}$ Glycerol und $3456 \mathrm{ml}$ destilliertem Wasser gemischt. Dazu wurde unter Rühren $252 \mathrm{~g}$ Trockenagar gegeben.

\section{Erhitzen im Wasserbad}

Beide Behälter wurden mit Aluminiumfolie (mit einem kleinen Loch zum Druckausgleich) abgedeckt und in einem Wasserbad von $90-100^{\circ} \mathrm{C}$ auf $85-90{ }^{\circ} \mathrm{C}$ erhitzt. Sobald die Lösungen eine Temperatur von $85^{\circ} \mathrm{C}$ erreichten, wurden sie durchmischt. Oberflächlicher Schaum wurde abgeschöpft, um Lufteinschlüsse zu vermeiden.

\section{Zusammenfügen der Lösungen}

Die beiden Lösungen (ca. 5 Liter Agar-Gemisch und 4 Liter Gelatine-Gel) wurden in einem Behälter von mindestens 10 Liter Fassungsvermögen zusammengegeben und verrührt. Unter regelmäßigem Umrühren wurde das Gemisch auf $50{ }^{\circ} \mathrm{C}$ abgekühlt und anschließend mit $32 \mathrm{~g}$ einer 37\%-Formaldehydlösung vermischt.

\section{Abdichtung mit Vaseline}

Um zusätzliche Lufteinschlüsse zu vermeiden, wurde das Phantom mit $1000 \mathrm{~g}$ geschmolzener Vaseline abgedichtet. Das gesamte Gemisch wurde über $24 \mathrm{~h}$ zum Abkühlen gestellt.

\section{Abdichtung mit Paraffin}

Es wurden $600 \mathrm{~g}$ Paraffin-Platelets (Surgipath, Paraplast, Tissue Embedding Medium) im Wasserbad zum Schmelzen gebracht und auf die ausgehärtete VaselineSchicht gegossen. 


\subsubsection{Durchführung der Phantommessungen zur Erfassung von Phasen- Offset-Fehlern}

Das statische Phantom wurde nach jeder Probandenmessung in dem Scanner positioniert (Abbildung 6). Die Messbedingungen (Schnittebene, Höhe des MRT-Tischs) wurden dabei konstant gehalten, um identische Voraussetzungen wie bei der zuvor erfolgten Probandenmessung zu erreichen. Das anteriore 16-Kanal-Spulenelement wurde auf dem Phantom positioniert. Der Phantomkörper übernahm somit nach der Probandenmessung die Rolle des menschlichen Thorax.

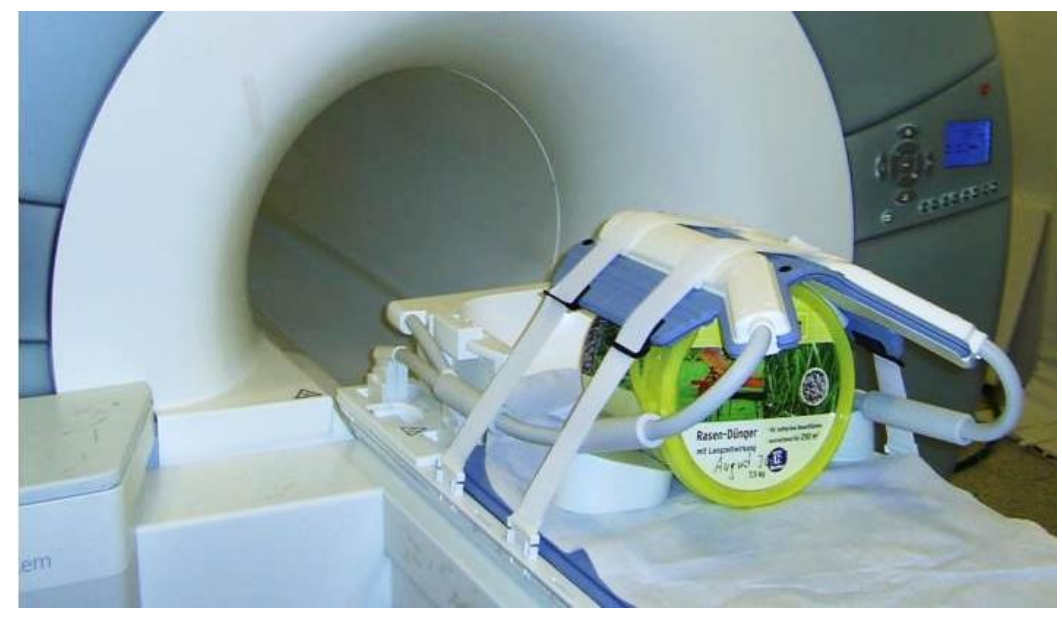

Abbildung 6: Vorbereitung der statischen Phantommessung. Auf dem Phantom (gelber Behälter) wurde die Thorax-Spule befestigt. An dem Phantom wurden alle vier MRT-Flussmessungen nach der Probandenmessung wiederholt.

Die konventionellen, EKG-synchronisierten CINE-Flussmessungen (CINE-FB, CINE$\mathrm{BH}$ ) und die Echtzeit-CMR-Flussmessungen (RT-CMR-Int, RT-CMR-Seq) erfolgten jeweils ein Mal an dem statischen Phantom. Entsprechend den In-VivoUntersuchungen wurden die Echtzeit-CMR-Flussmessungen für 15 Sekunden durchgeführt. Ein simuliertes EKG-Signal mit einer Herzfrequenz von 60 bpm diente den CINE-Flussmessungen zur Synchronisation und den Echtzeit-CMR-Flussmessungen zur Simulation aufeinanderfolgender Herzzyklen.

Die Auswertung erfolgte mit einer Prototype-Software (QFlow, MEDIS, Medical Imaging Systems BV, Leiden, Niederlande). Für die Analyse wurde jeweils die gespeicherte Kontur der entsprechenden ersten Probandenmessung (z. B. die Kontur der 
ersten CINE-FB Messung für die Messung der CINE-FB am statischen Phantom) verwendet (vgl. 2.1.2.2). Die über die entsprechende Querschnittfläche des Phantoms gemittelte Durchschnittsgeschwindigkeit wurde zur Erfassung des PhasenOffset Fehlers verwendet. Im Idealfall sollten sich geringe positive und negative Flusssignale gegeneinander aufheben. Eine Durchschnittsgeschwindigkeit von $0 \mathrm{~cm}$ $\mathrm{s}^{-1}$ entspräche schließlich einem Fehlen von Phasen-Offset-Fehlern.

\subsubsection{Statistik}

Die statistische Analyse wurde mit Hilfe von IBM SPSS Statistics Version 22 für Macintosh OS $X$ durchgeführt. Für den Vergleich von Phasen-Offset-Fehlern diente jeweils ein Wert der Durchschnittsgeschwindigkeit (CINE-PhasenkontrastFlussmessungen) bzw. der Mittelwert der Durchschnittsgeschwindigkeit aus 10 simulierten Herzschlägen (Echtzeit-CMR-Flussmessungen). Die Werte wurden - gemittelt über alle Phantommessungen - als Mittelwert \pm Standardabweichung angegeben. Entsprechend den Vorarbeiten von Gatehouse et al. wurden Werte der Durchschnittsgeschwindigkeit $>0,6 \mathrm{~cm} \mathrm{~s}^{-1}$ als signifikante Phasen-Offset-Fehler definiert (Gatehouse et al. 2010).

\subsubsection{In-Vivo-Evaluation der Echtzeit-CMR-Flussmessung}

Die Ethikkommission der Universitätsmedizin Göttingen bewilligte im Vorab die Durchführung der Studie. Das neu entwickelte Echtzeit-Verfahren wurde mit klinisch etablierten CINE-Flussmessungen verglichen. Im Zentrum standen dabei der Vergleich hämodynamischer Parameter (Schlagvolumen und Maximalgeschwindigkeit), der Vergleich der Auswertbarkeit der MRT-Flussmessungen und der Vergleich der Maximalgeschwindigkeit mit der Doppler-Echokardiographie.

Es wurden insgesamt 10 männliche Probanden ( $25 \pm 2$ Jahre [Mittelwert $\pm S D$ ], Spannbreite 20 - 30 Jahre; BMI $23 \pm 3$, Spannweite 20 - 28) von der Georg-AugustUniversität Göttingen rekrutiert und prospektiv in die Studie eingeschlossen. Alle Probanden waren zum Zeitpunkt der Untersuchung anamnestisch herzgesund und stellten sich freiwillig für die Studie zur Verfügung. 
Vor der MRT-Untersuchung erfolgte die Probandenaufklärung. Besonders eventuelle Kontraindikationen, die eine MRT Untersuchung verbieten würden (Herzschrittmacher, ferromagnetische Prothesen etc.), standen im Zentrum der Befragung. Aufgrund der engen Platzverhältnisse in dem MRT wurden die Probanden gezielt auf eine möglicherweise bestehende Klaustrophobie befragt. Neben einem mündlichen Gespräch erfolgte die Aufklärung anhand eines Aufklärungsbogens der NMR Forschungs $\mathrm{GmbH}$ am Max Planck Institut für biophysikalische Chemie sowie mit einer schriftlichen Probandenaufklärung des Instituts für Diagnostische und Interventionelle Radiologie der Universitätsmedizin Göttingen. Die Probanden wurden darin zusätzlich auf die Pseudonymisierung ihrer Daten hingewiesen.

Alle Messungen wurden in einem 3 Tesla MRT (Tim Trio, Siemens Healthcare, Erlangen, Deutschland) in den Gebäuden der NMR Forschungs GmbH am Max Planck Institut für biophysikalische Chemie in Göttingen durchgeführt. Die Probanden wurden in Rückenlage auf der Liege des MRT positioniert. Anschließend wurde ein MRT-taugliches Vektor-Elektrokardiogramm (EKG) mit drei Ableitungen entsprechend den Herstellerempfehlungen angelegt. Um die Übertragung des EKG-Signals zu sichern, wurde die Vorrichtung des EKG-Senders in eine Silikonapparatur eingebettet. Aufgrund des hohen Lärmpegels während der MRT Untersuchung erhielten die Probanden einen Ohrenschutz. Für die MR-Signalaufnahme wurde eine standardmäßige 32-Kanal-Herzspule verwendet, die sich aus einem anterioren und posterioren 16-Kanal-Element zusammensetzt. Das posteriore Element wurde in die Patientenliege eingefasst, während die anteriore Spule auf dem Thorax platziert wurde (Abbildung 7). Eine Alarmklingel ermöglichte den Probanden sich bei eventuell auftretenden Problemen während der MRT-Untersuchung bemerkbar zu machen. Zusätzlich war die Person im MRT über ein Mikrophon und Lautsprecher mit dem MRT-Vorraum in Kontakt.

Für den Vergleich der MRT-Flussmessungen wurde als Messposition die aufsteigende Aorta (AAo) ausgewählt. Dazu wurde eine Schnittebene gewählt, die senkrecht zur AAo lag (sog. Through-plane-Messung). Aus dem sagittalen Localizer (multislice radial fast low angle shot [FLASH]) wurde eine Schnitteben ermittelt, welche die rechte Pulmonalarterie im Querschnitt und die AAo im Längsschnitt zeigte. Aus dieser Ebene wurde die endgültige Messebene definiert, die auf Höhe der rechten Pulmonalarterie die AAo senkrecht zur Flussrichtung schnitt (Abbildung 8). 

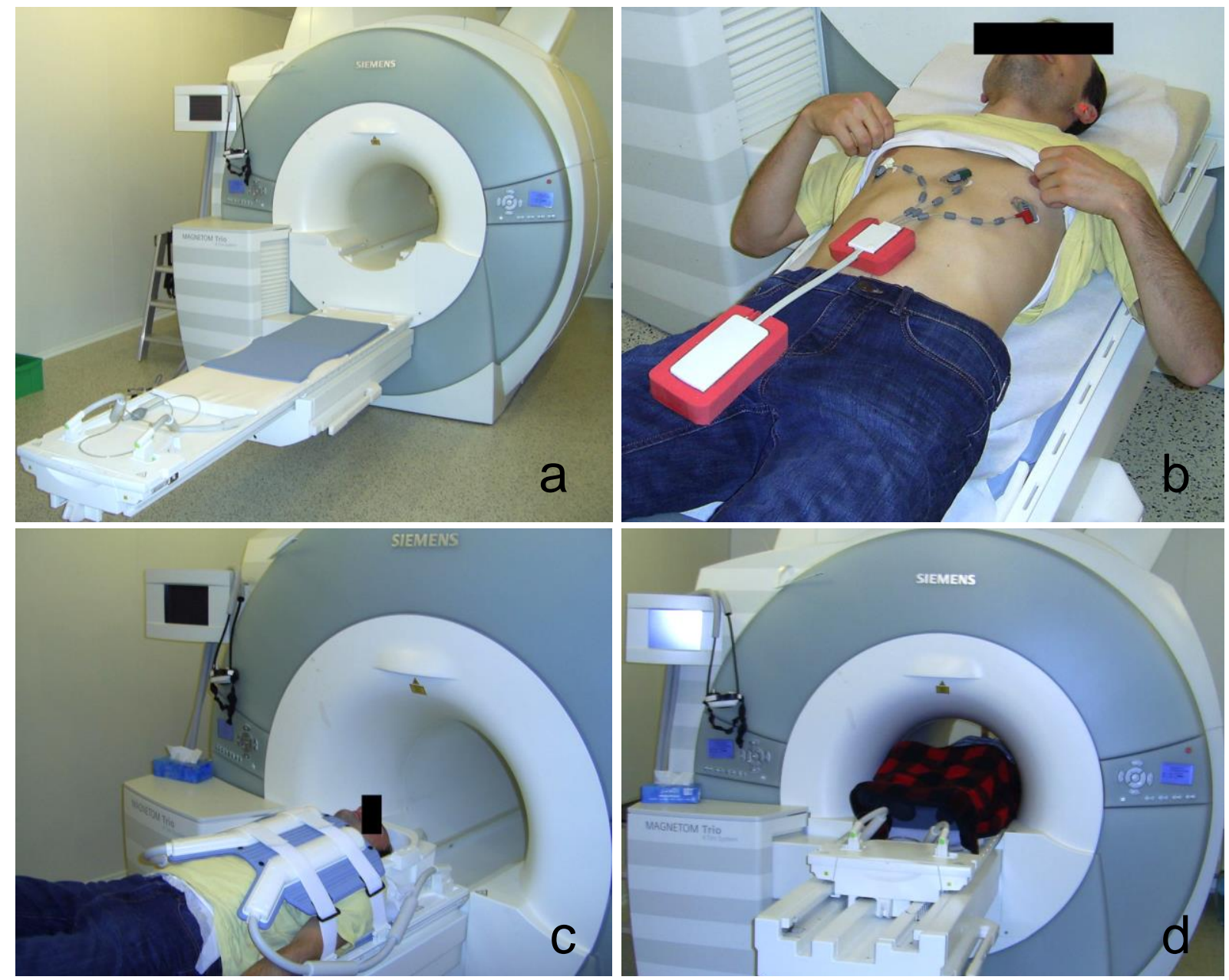

Abbildung 7: Vorbereitung eines Probanden. a: 3 T MRT (TIM Trio, Siemens Healthcare, Erlangen, Deutschland). b: Anlage der EKG-Elektroden. Silikonapparatur um den EKG-Sender (rot) c: Proband mit sichtbarem anteriorem 16-Kanal-Element der 32-Kanal-Herzspule auf dem Thorax. d: Proband während der Messung im MRT.

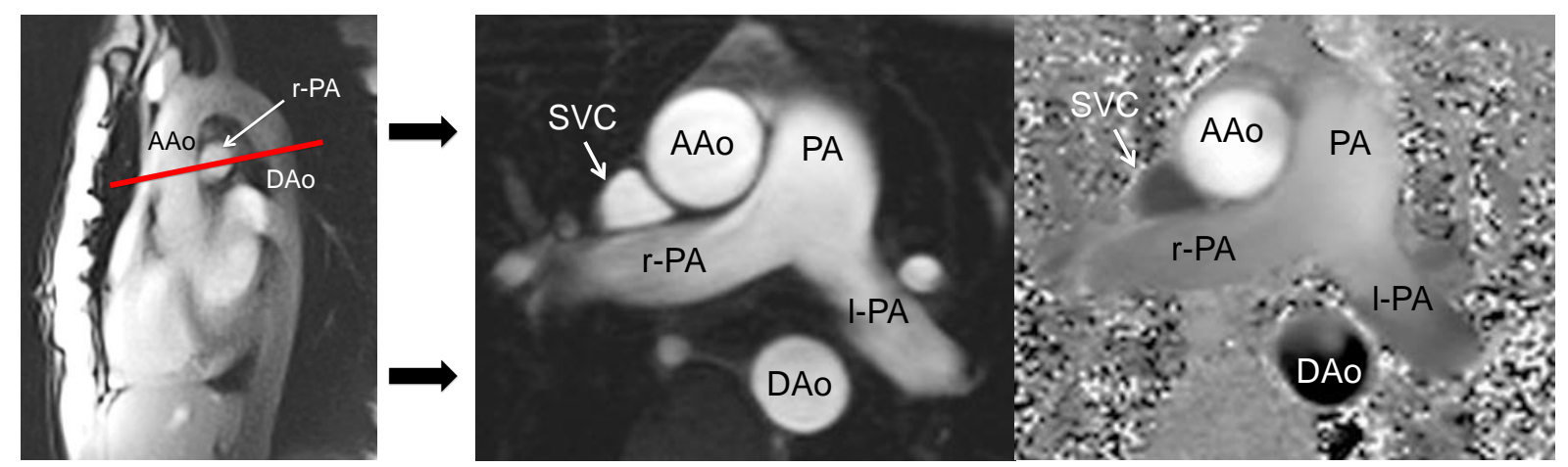

Abbildung 8: Ermittlung der Messposition. Aus dem sagittalen Localizer (links) wurde eine Schnittebene auf Höhe der rechten Pulmonalarterie (r-PA) erzeugt, die senkrecht zur Aorta ascendens (AAo) lag (rote Linie). Endgültige Messebene (rechts) mit Betrags-und Phasenbild. Aorta descendens (DAo), Vena cava superior (SVC), linke Pulmonalarterie (I-PA), Truncus pulmonalis (PA). 


\subsubsection{Vergleich hämodynamischer Parameter}

Für den Vergleich der verschiedenen MRT-Flussmessungen wurden als quantitative Parameter das Schlagvolumen (Flussvolumen pro Herzschlag) und die Maximalgeschwindigkeit (Single-Pixel) verwendet. Es wurden 2 Varianten der CINEFlussmessung mit 2 Varianten der Echtzeit-Flussmessung verwendet. Die vier MRTVerfahren wurden bei jedem Probanden insgesamt drei Mal in randomisierter Reihenfolge wiederholt.

\section{Echtzeit-CMR-Flussmessung}

Die Grundlage der verwendeten Echtzeit-Methode bildet eine FLASH-Sequenz mit einem bewegungskompensierenden Schichtselektionsgradienten und einem flusskodierenden bipolaren Gradienten (Joseph et al. 2014). Die Echtzeit-CMRFlussmessung ermöglichte die kontinuierliche Aufnahme von T1-gewichteten Bildern mit einer zeitlichen Auflösung von 40 ms und einer nominellen Ortsauflösung von 1,3 $x 1,3 \mathrm{~mm}^{2}$ (Tabelle 2). Die k-Raum-Abtastung erfolgte radial mit Hilfe von 7 Speichen. Die Echtzeit-CMR-Flussmessung wurde in zwei technischen Varianten angewendet, die sich lediglich in der zeitlichen Anordnung der flusskodierenden bipolaren Gradienten unterschieden. Diese Gradienten wurden innerhalb der zwei Messungen entweder verschachtelt (RT-CMR-Int) oder sequentiell (RT-CMR-Seq) angeordnet. Im Rahmen der Studie sollten beide Varianten getestet und mit den klinisch etablierten CINE-Phasenkontrast-Flussmessungen verglichen werden. Die Echtzeit-CMRFlussmessungen sind weder auf eine EKG-Triggerung noch auf eine Atemkompensation angewiesen. Es wurde dennoch ein EKG-Signal aufgezeichnet, um Zeitmarken (R-Zacke) zu setzen. Diese Zeitmarken dienten der automatischen Unterteilung in einzelne Herzzyklen in der späteren Funktionsanalyse. Für die Rekonstruktion der Echtzeit-Daten wurde ein Verfahren mittels iterativ regularisierter, nichtlinearer Inversion (NLINV) entwickelt (Uecker et al. 2010 a). Der NLINV-RekonstruktionsMechanismus wurde auf einem bypass-Computersystem mit 8 parallel geschalteten Graphikkarten implementiert (GPUs, GeForce GTX 580, Nvidia, Santa Clara, California), das eine Online-Rekonstruktion erlaubt. Dadurch konnten die rekonstruierten Bilder bereits während der Flussmessung mit einer kurzen zeitlichen Verzögerung von ca. 2 Sekunden auf den Monitoren der MRT-Workstation betrachtet werde. Alle Echtzeit-CMR-Aufnahmen wurden in freier Atmung für eine Dauer von 15 Sekunden 
durchgeführt. Die akquirierten Datensets umfassten je nach Herzfrequenz entsprechend 12 bis 15 Herzschläge.

\section{Konventionelle CINE-Phasenkontrast-Flussmessung}

Die beiden abgewandelten Formen der Echtzeit-CMR-Flussmessung wurden mit zwei konventionellen, retrospektiv EKG-synchronisierten CINE-PhasenkontrastFlussmessungen verglichen. Dazu wurde zum einen eine CINE-PhasenkontrastFlussmessung verwendet, die eine Messung in freier Atmung (CINE-FB) erlaubt, zum anderen eine CINE-Phasenkontrast-Flussmessung, die in Atemstillstand (CINE-BH) durchgeführt werden musste. Die grundlegenden Messparameter waren für beide Sequenztypen identisch: Schichtdicke $6 \mathrm{~mm}$, Flip-Winkel $25^{\circ}, \mathrm{VENC}=200 \mathrm{~cm} \mathrm{~s}^{-1}$. Die nominelle Ortsauflösung und die zeitliche Auflösung waren bei CINE-FB höher als bei CINE-BH (Tabelle 2). Die Daten wurden mittels paralleler Bildgebung (GRAPPA, 24 Referenzlinien) rekonstruiert. Die Zeit für eine gesamte Flussmessung mit CINE-FB lag bei 3,5 Minuten, während CINE-BH ca. 15 Sekunden benötigte.

\subsubsection{Vergleich der Auswertbarkeit}

Alle derzeit kommerziell erwerbbaren Analyse-Softwares zur Auswertung von MRTFlussmessungen erlauben lediglich die Bildanzahl einer konventionellen CINEPhasenkontrast-Flussmessung (20-30 Bildpaare) einzulesen und zu analysieren. Die Echtzeit-CMR-Flussmessung ergibt bei 15-sekündlichen Messungen (zeitliche Auflösung $40 \mathrm{~ms}$ ) jedoch 375 Bildpaare. Aus diesem Grund wurde eine PrototypeSoftware auf der Grundlage der QFlow-Software (MEDIS, Medical Imaging Systems BV, Leiden, Niederlande) entwickelt. Diese Prototype-Software ermöglicht das Einladen von bis zu 1000 Bildpaaren und erlaubt eine automatische Unterteilung der Echtzeit-Daten in einzelne Herzzyklen. Dabei orientiert sich der Algorithmus an den Zeitmarken, die durch die R-Zacke des miterfassten EKG-Signals gesetzt wurden. Die Software unterliegt einem semiautomatischen Segmentationsalgorithmus. Um eine Bildfolge zu analysieren, wurde anhand eines Magnitudenbildes eine initiale ROI um die AAo definiert. Davon ausgehend wurden die Konturen in allen Phasen mittels automatischer Erkennung ergänzt. Alle Konturen wurden anschließend visuell kontrolliert und, wenn erforderlich, manuell korrigiert. 
Tabelle 2: Sequenzparameter der konventionellen CINE-Flussmessungen und der Echtzeit(RT)-CMR-Flussmessungen

\begin{tabular}{|c|c|c|c|c|}
\hline Parameter & CINE-FB & CINE-BH & RT-CMR-Int & RT-CMR-Seq \\
\hline Ortsauflösung & $\begin{array}{c}1,3 \times 1,3 \times 6 \\
\mathrm{~mm}^{3}\end{array}$ & $\begin{array}{c}1,7 \times 1,7 \times 6 \\
\mathrm{~mm}^{3}\end{array}$ & $\begin{array}{c}1,3 \times 1,3 \times 6 \\
\mathrm{~mm}^{3}\end{array}$ & $\begin{array}{c}1,3 \times 1,3 \times 6 \\
\mathrm{~mm}^{3}\end{array}$ \\
\hline FOV & $320 \times 240 \mathrm{~mm}^{2}$ & $320 \times 220 \mathrm{~mm}^{2}$ & $192 \times 192 \mathrm{~mm}^{2}$ & $192 \times 192 \mathrm{~mm}^{2}$ \\
\hline $\begin{array}{l}\text { TR / TE / Flip- } \\
\text { Winkel }\end{array}$ & $\begin{array}{c}20,05 / 2,18 \mathrm{~ms} \\
/ 25^{\circ}\end{array}$ & $\begin{array}{c}46,90 / 1,92 \mathrm{~ms} \\
/ 25^{\circ}\end{array}$ & $\begin{array}{c}2,86 / 1,93 \mathrm{~ms} / \\
10^{\circ}\end{array}$ & $\begin{array}{c}2,86 / 1,93 \mathrm{~ms} / \\
10^{\circ}\end{array}$ \\
\hline $\operatorname{VENC}\left(\mathrm{cm} \mathrm{s}^{-1}\right)$ & 200 & 200 & 200 & 200 \\
\hline Zeitauflösung & $\begin{array}{c}20,05 \mathrm{~ms} \\
\text { interpoliert }\end{array}$ & $\begin{array}{c}46,90 \mathrm{~ms} \\
\text { interpoliert }\end{array}$ & $\begin{array}{l}40,0 \mathrm{~ms} \\
\text { real }\end{array}$ & $\begin{array}{l}40,0 \mathrm{~ms} \\
\text { real }\end{array}$ \\
\hline $\begin{array}{l}\text { Gradientenko- } \\
\text { dierung }\end{array}$ & verschachtelt & verschachtelt & verschachtelt & sequentiell \\
\hline $\begin{array}{l}\text { EKG-Synchro- } \\
\text { nisation }\end{array}$ & retrospektiv & retrospektiv & - & - \\
\hline $\begin{array}{l}\text { Atem- } \\
\text { kompensation }\end{array}$ & freie Atmung & Atemstillstand & nicht notwendig & nicht notwendig \\
\hline $\begin{array}{l}\text { k-Raum- } \\
\text { Abtastung }\end{array}$ & $\begin{array}{l}\text { kartesisch (24 } \\
\text { Referenzlinien) }\end{array}$ & $\begin{array}{l}\text { kartesisch (24 } \\
\text { Referenzlinien) }\end{array}$ & $\begin{array}{c}\text { radial } \\
\text { (7 Speichen) }\end{array}$ & $\begin{array}{c}\text { radial } \\
\text { (7 Speichen) }\end{array}$ \\
\hline Rekonstruktion & GRAPPA* & GRAPPA* & NLINV $^{\dagger}$ & NLINV $^{\dagger}$ \\
\hline $\begin{array}{l}\text { Anzahl rekon- } \\
\text { struierter Pha- } \\
\text { sen pro } \\
\text { Herzzyklus }\end{array}$ & 30 & 20 & $\begin{array}{c}\text { abhängig von } \\
\text { Herzfrequenz: } \\
\quad 15-30\end{array}$ & $\begin{array}{l}\text { abhängig von } \\
\text { Herzfrequenz: } \\
15-30\end{array}$ \\
\hline
\end{tabular}


Die QFlow-Prototype Software bietet für die Korrektur der Konturen entsprechende Werkzeuge an.

Um die Auswertbarkeit der Echtzeit-und CINE-Daten zu bewerten, wurde die Anzahl an Phasen gezählt, deren ROI manuell korrigiert werden musste. Des Weiteren wurde für jede Auswertung die Zeit bestimmt, die für die Korrektur erforderlich war. Um die Interobserver-Variabilität zu minimieren, wurde im Konsensus-Reading-Verfahren ausgewertet, sodass zwei Personen gleichzeitig an der Auswertung beteiligt waren. Die manuellen Konturänderungen wurden in gemeinsamer Übereinstimmung wechselseitig von einer Person vorgenommen, während die zweite Person die Anzahl korrigierter Konturen zählte und die Korrekturzeit stoppte. Die Ergebnisse der funktionellen Flussparameter wurden sowohl vor als auch nach der manuellen Konturkorrektur notiert. Dadurch sollte der Einfluss der manuellen Korrektur auf die Parameter Schlagvolumen und Maximalgeschwindigkeit überprüft werden.

\subsubsection{Vergleich mit der Doppler-Echokardiographie}

Nachdem die 10 Probanden an der MRT-Untersuchung teilgenommen hatten, wurde bei innen zusätzlich die Maximalgeschwindigkeit mittels Continous-Wave (CW)Doppler $(1,8 \mathrm{MHz})$ in der transthorakalen Echokardiographie im Herzzentrum der Universitätsmedizin Göttingen (Gerätetyp: iE33, Fa. Philips, Niederlande) bestimmt. Alle Messungen wurden von einem Untersucher mit über 20 Jahren Praxiserfahrung auf dem Gebiet der Echokardiographie durchgeführt. Für den Vergleich mit den MRT-Flussmessungen wurde mindestens eins von vier Schallfenstern angestrebt: apikaler Fünf- oder Drei-Kammerblick, parasternale lange Achse oder ein Schallfenster aus suprasternaler Blickrichtung. Dabei zielten alle Schallfenster auf die CWDoppler-Flussmessung entlang der Aortenklappe ab.

Die Bilddaten wurden zuerst im PACS-System der Universitätsmedizin Göttingen digital gespeichert und anschließend mit einer kardiologischen Analyse-Software ausgewertet (2D Cardiac Performance Analysis, TomTec Imaging System, München, Deutschland). Dazu wurde um das systolische Echosignal eine Kontur gelegt, dessen äußerste Spitze der gemessenen Maximalgeschwindigkeit entsprach. In jedem Ultraschallfenster wurde der CW-Doppler über eine Schleife von 3 Herzzyklen aufge- 
nommen. Für den Vergleich mit den MRT-Flussmessungen dienten die drei Schleifen mit den höchsten Maximalgeschwindigkeiten.

\subsubsection{Statistik}

Die statistische Analyse wurde mit Hilfe von Microsoft Excel 2011 und IBM SPSS Statistics Version 22 für Macintosh OS X durchgeführt. Der Shapiro-Wilk Test wurde verwendet um die Normalerteilung der Daten zu überprüfen.

\section{Vergleich hämodynamischer Parameter}

Werte des Schlagvolumens und der Maximalgeschwindigkeit wurden für EchtzeitCMR-Flussmessungen aus 3 Wiederholungen mit je 10 Herzzyklen (insgesamt 30 Herzzyklen) und für CINE-Phasenkontrast-Flussmessungen aus 3 Wiederholungen mit je einem interpoliertem Herzzyklus (insgesamt 3 Herzzyklen) als Mittelwert \pm Standardabweichung (SD) für jeden Probanden ermittelt. Es wurden PearsonKorrelationen und Bland-Altman-Analysen in prozentualer Transformation für wiederholende Messungen zur Ermittlung der Übereinstimmung durchgeführt (Bland and Altman 1999). Die Werte wurden mit einem gepaarten, zweiseitigen t-Test verglichen. Alle $p$-Werte $<0,05$ wurden als statistisch signifikant definiert.

\section{Vergleich der Auswertbarkeit}

Die Zeit für die manuelle Korrektur der MRT-Flussmessungen in der Funktionsanalyse wurde gestoppt und als Mittelwert \pm SD (10 Probanden, 3 Wiederholungen) angegeben. Die Anzahl korrekturbedürttiger Phasen wurde gezählt und in absoluten Zahlen als Mittelwert \pm SD sowie als prozentualer Anteil in Bezug zur Gesamtphasenanzahl angegeben. Der Mittelwert \pm SD (10 Probanden, 3 Wiederholungen) des Schlagvolumens und der Maximalgeschwindigkeit wurden sowohl vor der manuellen Korrektur als auch nach der Korrektur ermittelt. Die Werte wurden mit einem gepaarten, zweiseitigen $t-T e s t$ verglichen. Alle $p$-Werte $<0,05$ wurden als statistisch signifikant definiert.

\section{Vergleich mit der Doppler-Echokardiographie}

Werte der Maximalgeschwindigkeit wurden für Echtzeit-CMR-Flussmessungen aus 3 Wiederholungen mit je 10 Herzzyklen (insgesamt 30 Herzzyklen), für CINEPhasenkontrast-Flussmessungen aus 3 Wiederholungen mit je einem interpoliertem 
Herzzyklus (insgesamt 3 Herzzyklen) und für Doppler-Flussmessungen aus 3 Wiederholungen mit je 3 Herzzyklen (insgesamt 9 Herzzyklen) als Mittelwert \pm SD für jeden Probanden angegeben. Es wurden Pearson-Korrelationen und Bland-AltmanAnalysen in prozentualer Transformation für wiederholende Messungen zur Ermittlung der Übereinstimmung durchgeführt (Bland and Altman 1999). Die Werte wurden mit einem gepaarten, zweiseitigen $t-T e s t$ verglichen. Alle $p$-Werte $<0,05$ wurden als statistisch signifikant definiert.

\subsection{Anwendung der Echtzeit-CMR-Flussmessung mit dem Valsalva-Manöver als physiologischem Stressor}

Die Ethikkommission der Universitätsmedizin Göttingen bewilligte vorab die Durchführung der Studie. Für die Anwendung der Echtzeit-CMR-Flussmessungen unter dem Valsalva-Manöver wurde ein zweites Studienkollektiv rekrutiert. 20 freiwillige, anamnestisch herzgesunde Probanden (12 männlich, 8 weiblich) im Alter von 33,9 \pm 12,8 Jahren (Spannweite 22 - 59 Jahre) mit einem BMl von 22,8 $\pm 2,6 \mathrm{~kg} \mathrm{~m}^{-2}$ nahmen an der Studie teil. Die Messungen fanden in der biomedizinischen NMR Forschungs $\mathrm{GmbH}$ am Max Planck Institut für biophysikalische Chemie in Göttingen statt. Die grundlegende Geräteausstattung (3 T MRT, 32-Kanal-Herzspule), Aufklärung und Vorbereitung (Lagerung, EKG, Gehörschutz) der Probanden wurde identisch vorgenommen wie in der primär durchgeführten Studie (vgl. 2.1.2).

\subsubsection{Experimenteller Versuchsaufbau und Versuchsdurchführung}

Zur reproduzierbaren Durchführung der Valsalva-Manöver wurden die Probanden aufgefordert, während der Echtzeit-CMR-Flussmessung in ein Mundstück (Oxford Tracheal Tube 7,0) zu pressen, das über einen druckresistenten PVC-Schlauch auBerhalb des MRT-Raumes mit einem digitalen Manometer (GMSD 2 BR, Greisinger electronic $\mathrm{GmbH}$, Regenstauf, Deutschland) verbunden wurde. Über eine LCD-Brille wurde dem Probanden ein animierter Timer präsentiert (Abbildung 9). Die Präsentation wurde zusammen mit der Echtzeit-CMR-Flussmessung (RT-CMR-Seq) gestartet. Ein Druck von $40 \mathrm{mmHg}$ erwies sich in früheren Studien als besonders effizient, um hämodynamische Änderungen hervorzurufen (Robertson et al. 1977). 

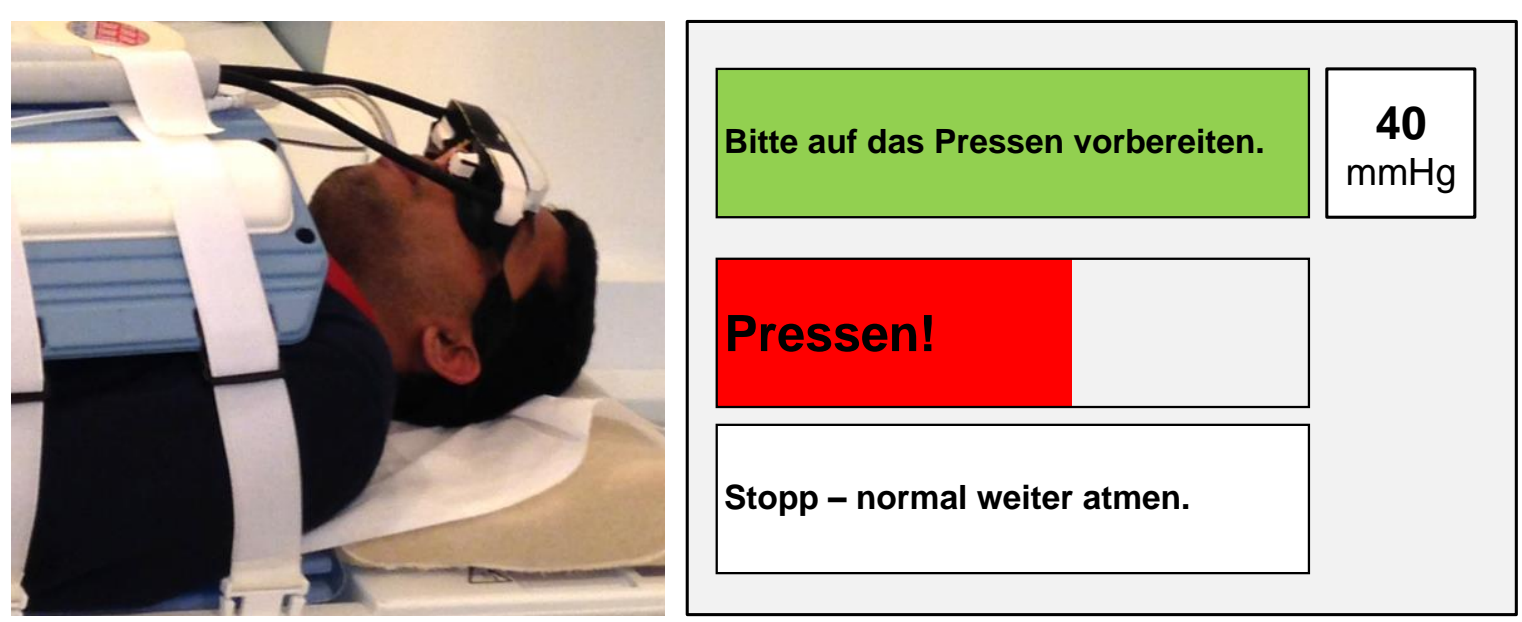

Abbildung 9: Versuchsaufbau der Echtzeit-CMR-Flussmessung unter dem ValsalvaManöver. Links: Proband mit 32-Kanal Herzspule, Mundstück und LCD-Brille. Rechts: Präsentation, die dem Probanden während der Messung über die LCD-Brille präsentiert wurde. Die Balken wurden bewegungsanimiert und "liefen" während der Flussmessung von links nach rechts. Oben rechts: aktuell erreichter Druckwert.

Während der MRT-Flussmessung wurde über die LCD-Brille der aktuell erreichte Druck als Biofeedback angezeigt. Die Echtzeit-CMR-Flussmessungen wurden für 40 Sekunden durchgeführt. Der Blutfluss wurde dabei 10 Sekunden in Ruheatmung, 10 Sekunden unter dem Valsalva-Manöver und 20 Sekunden in der Erholungsphase gemessen. Während des Valsalva-Manövers sollte der Proband einen Druck von 40 $\mathrm{mmHg}$ aufrecht halten. Vor der MRT-Untersuchung wurde das Valsalva-Manöver im MRT-Vorraum mehrfach geübt. Die 40 sekündlichen Messungen wurden insgesamt 3 Mal wiederholt. Zwischen den Messungen wurde zur Erholung eine 5-minütige Pause eingelegt. Das Flussvolumen pro Herzschlag $(\mathrm{ml})$ und die Gefäßquerschnittsfläche $\left(\mathrm{mm}^{2}\right)$ wurde während des Valsalva-Manövers simultan in der oberen Hohlvene (SVC) und der aufsteigenden Aorta (AAo) bestimmt. Dazu wurde eine Schnittebene gewählt, die beide Gefäße senkrecht zur Blutflussrichtung schneidet (vgl. 2.1.2). Zur Einstellung der Schnittebene wurden Localizer (multislice radial FLASH) sowohl in Ruheatmung als auch unter dem Valsalva-Manöver angefertigt. Die Messposition wurde entsprechend der physiologischen Verlagerung der mediastinalen Organe während des Valsalva-Manövers, wenn erforderlich, entsprechend angepasst. Die Analyse der Echtzeit-Daten wurde mit der QFlow-Prototype Software (MEDIS, Medical Imaging Systems BV, Leiden, Niederlande) vorgenommen. Dazu wurden zwei initiale ROI definiert - eine um die SVC und eine um die AAo. Beide Konturen wur- 
den automatisch auf alle Phasen übertragen und, wenn erforderlich, manuell korrigiert (vgl. 2.1.2.2).

\subsubsection{Statistik}

Die statistische Analyse wurde mit Hilfe von Microsoft Excel 2011 und GraphPad Prism Version 6.0d für Macinstosh OS X durchgeführt. Die Blutflussprofile der 40sekündlichen Echtzeit-CMR-Flussmessungen wurden modifiziert nach Parisi et al. in 5 Phasen eingeteilt (Parisi et al. 1976):

- Phase 1 (normale Atmung): Alle Herzzyklen während den ersten 10 Sekunden.

- Phase 2 (frühe Anspannung): Erste 4 Herzzyklen eines Valsalva-Manövers.

- Phase 3 (späte Anspannung): Letzte 4 Herzzyklen eines Valsalva-Manövers.

- Phase 4 (frühe Erholung): Erste 3 Herzzyklen nach dem Valsalva-Manöver.

- Phase 5 (späte Erholung): Alle Herzzyklen bis zum Ende der Messung.

Für jede dieser Phase wurde ein Mittelwert \pm Standardabweichung der Parameter Flussvolumen pro Herzschlag $(\mathrm{ml})$ und Gefäßquerschnittsfläche $\left(\mathrm{mm}^{2}\right)$ für die SVC und AoA ermittelt. Neben der Angabe absoluter Flusswerte wurden die Werte der Phasen $2-5$ zusätzlich in prozentuale Relation zu den Werten aus Phase 1 (normale Atmung) gesetzt. Die Werte wurden über 20 Probanden und 3 Wiederholungsmessungen gemittelt (insgesamt 60 Valsalva-Manöver). Falls ein Proband nicht in der Lage war den Zieldruck von $40 \mathrm{mmHg}$ zu erreichen, wurde die Messung von der statistischen Analyse ausgeschlossen. Durch die Anspannung der Atemmuskulatur während eines Valsalva-Manövers konnten Fehler in der EKG-Übertragung auftreten. Einzelne Phasen des Valsalva-Manövers, in denen keine adäquate EKG-Ableitung stattfinden konnte, wurden ebenfalls von der statistischen Analyse ausgeschlossen.

Der d'Agostino und Pearson's omnibus Test wurde zur Überprüfung einer Normalverteilung der Daten verwendet. Eine einfaktorielle Varianzanalyse für wiederholende Messungen wurde mit Greenhouse-Korrektur - gefolgt von einem Tukey's-Test für mehrfache Vergleiche - durchgeführt, um Änderungen der hämodynamischen Parameter zwischen den unterschiedlichen Phasen des Valsalva-Manövers festzustellen. Alle $p$-Werte $<0,05$ wurden als statistisch signifikante Unterschiede definiert. 


\section{Ergebnisse}

\subsection{Vergleich von Echtzeit-CMR-Flussmessung und CINE-Flussmessung}

Der zeitliche Rahmen, einschließlich Aufklärung und Vorbereitung einer MRTProbandenuntersuchung, lag bei 30 bis 40 Minuten. Die Messung am statischen Phantom dauerte ca. 5 bis 10 Minuten. Die Doppler-echokardiographischen Untersuchungen nahmen durchschnittlich 10 bis 15 Minuten in Anspruch. Die im Folgenden beschriebenen Ergebnisse wurden zum Teil bereits unter Joseph 2013 und Joseph et al. 2014 publiziert.

\subsubsection{In-Vitro-Evaluation der MRT-Flussmessungen zum Auftreten von Pha- sen-Offset-Fehlern}

Die Messungen am statischen Phantom konnten in allen Fällen erfolgreich durchgeführt werden (Abbildung 10). Die Höhe an Phasen-Offset-Artefakten war bei allen verwendeten MRT-Flussmessungen gering. CINE-BH und die neu entwickelten Echtzeit-CMR-Flussmessungen (RT-CMR-Int, RT-CMR-Seq) zeigten in der Phantommessung Durchschnittsgeschwindigkeiten, die im Mittel unter $0,6 \mathrm{~cm} \mathrm{~s}^{-1}$ lagen. CINE-FB zeigte im Vergleich den höchsten Anteil an Phase-Offset-Artefakten. Die Standardabweichungen der Durchschnittsgeschwindigkeit waren bei den EchtzeitCMR-Flussmessungen im Vergleich zu den CINE-Flussmessungen geringer (Tabelle 3).

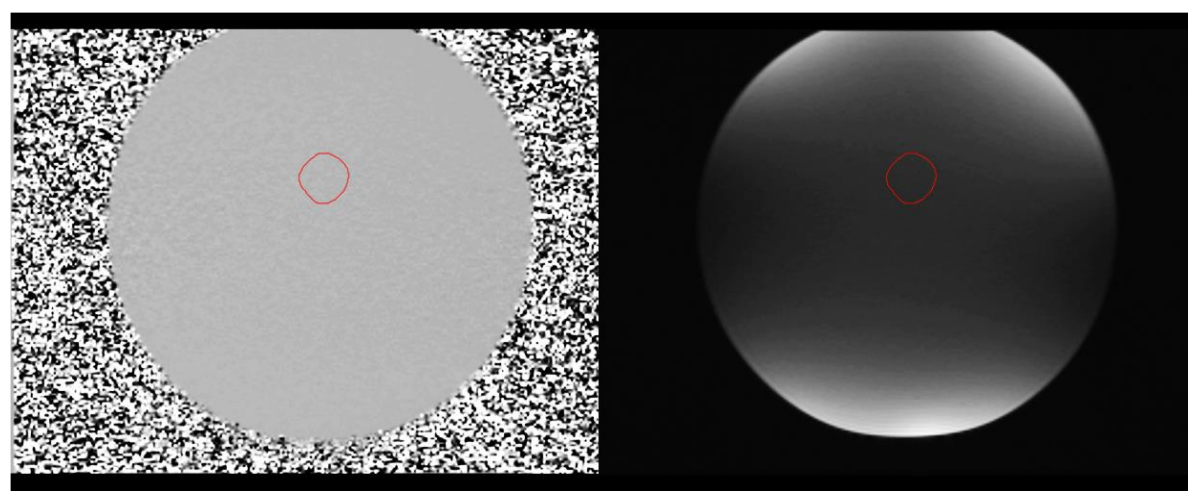

Abbildung 10: Statische Phantommessung zur Ermittlung von Phasen-OffsetFehlern. Links: Phasenbild. Rechts: Betragsbild. Verwendete Sequenz: CINE-FB. ROI (rot) entspricht der Gefäßkontur der ersten CINE-FB Messung des zuvor gescannten Probanden. 
Tabelle 3: Durchschnittsgeschwindigkeit der Phantommessungen (Mittelwert \pm Standardabweichung). Werte resultieren für CINE-Flussmessungen aus 10 Messungen mit einem interpolierten Herzzyklus, für Echtzeit(RT)-CMR-Flussmessungen aus 10 Messungen mit 10 Herzzyklen.

\section{Durchschnittsgeschwindigkeit $\left(\mathrm{cm} \mathrm{s}^{-1}\right)$}

$\begin{array}{lc}\text { CINE-FB } & 1,10 \pm 0,35 \\ \text { CINE-BH } & -0,12 \pm 0,17 \\ \text { RT-CMR-Int } & 0,07 \pm 0,03 \\ \text { RT-CMR-Seq } & 0,05 \pm 0,02\end{array}$

\subsubsection{In-Vivo-Evaluation der Echtzeit-CMR-Flussmessung}

Die rekonstruierten Magnituden- und Phasenbilder der Echtzeit-CMRFlussmessungen zeigten eine klare Darstellung und Abgrenzbarkeit der großen thorakalen Gefäße. Die Bilder der CINE-Phasenkontrast-Flussmessung waren von geringen Ghosting-Artefakten begleitet. Die Echtzeit-CMR-Flussmessungen (RT-CMRInt, RT-CMR-Seq) zeigten geringe Streifenartefakte in maximaler Systole. Während der Diastole tendierten die Echtzeit-Sequenzen zu einer visuell besseren Bildqualität und Abgrenzbarkeit der Gefäßwände der großen thorakalen Gefäße als die CINEPhasenkontrast-Flussmessungen (Abbildung 11).

\subsubsection{Vergleich hämodynamischer Parameter}

Die Echtzeit-CMR-Flussmessungen ermöglichten die Darstellung von Flussprofilen sukzessiver Herzzyklen. Die kontinuierliche Datenakquisition über multiple Herzschläge zeigte in regelmäßigen Abständen von 3-4 Herzschlägen zyklische Variationen des Schlagvolumens und der Maximalgeschwindigkeit in der Aorta ascendes (Abbildung 12). Das Schlagvolumen zeigte im dargestellten Beispiel innerhalb von 10 Herzzyklen Änderungen zwischen 99,5 ml und 114,2 ml (Mittelwert \pm SD: 106,5 \pm 4,7 ml), während die Maximalgeschwindigkeit in zwei direkt aufeinander folgenden Herzzyklen zwischen $101 \mathrm{~cm} \mathrm{~s}^{-1}$ und $85 \mathrm{~cm} \mathrm{~s}^{-1}$ (Abbildung 12 rechts; Herzzyklus 3 auf 4) variierte. 


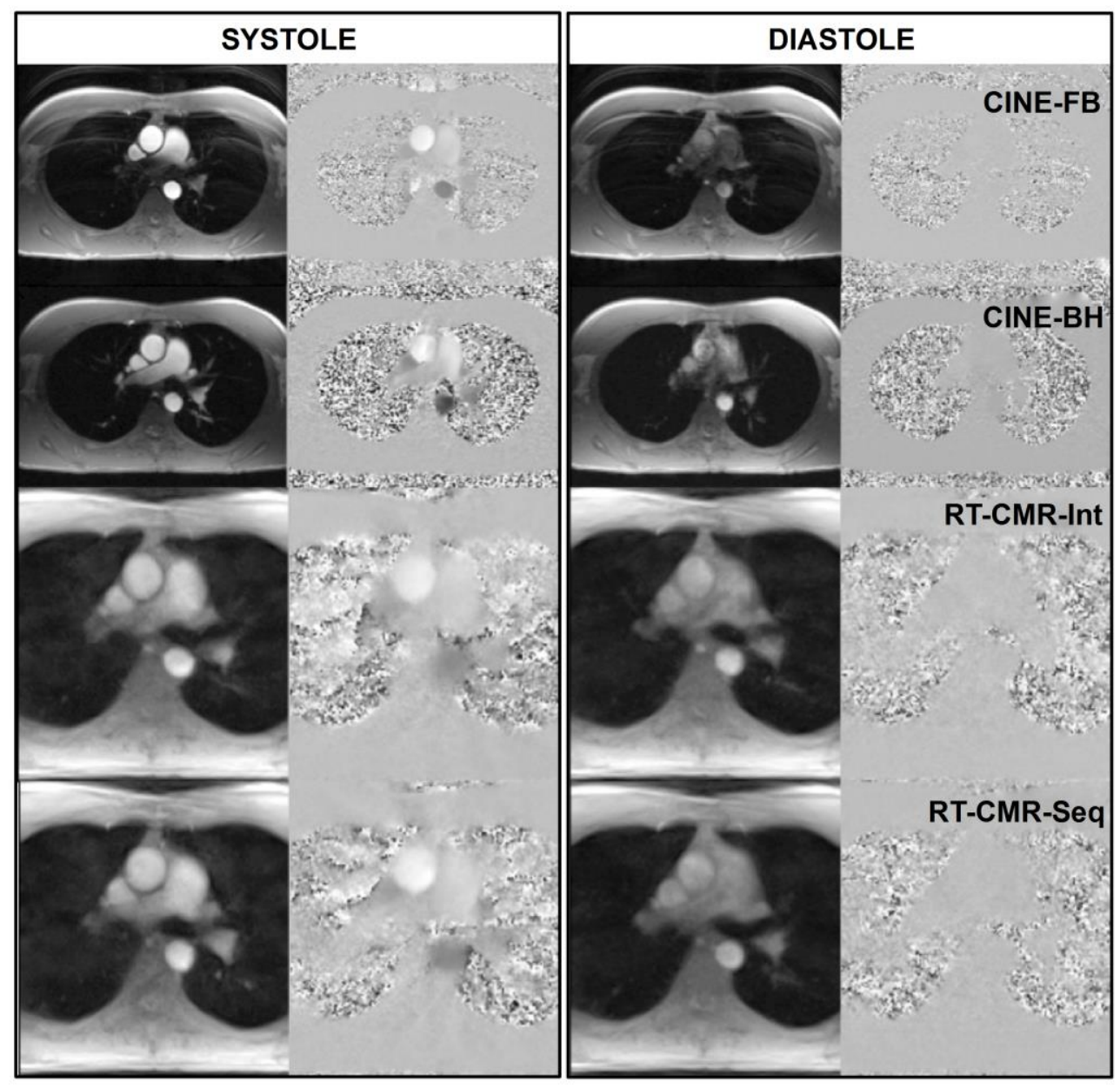

Abbildung 11: Bildqualität im Vergleich. Betrags- und Phasenbilder in Systole (links) und Diastole (rechts) eines Probanden.
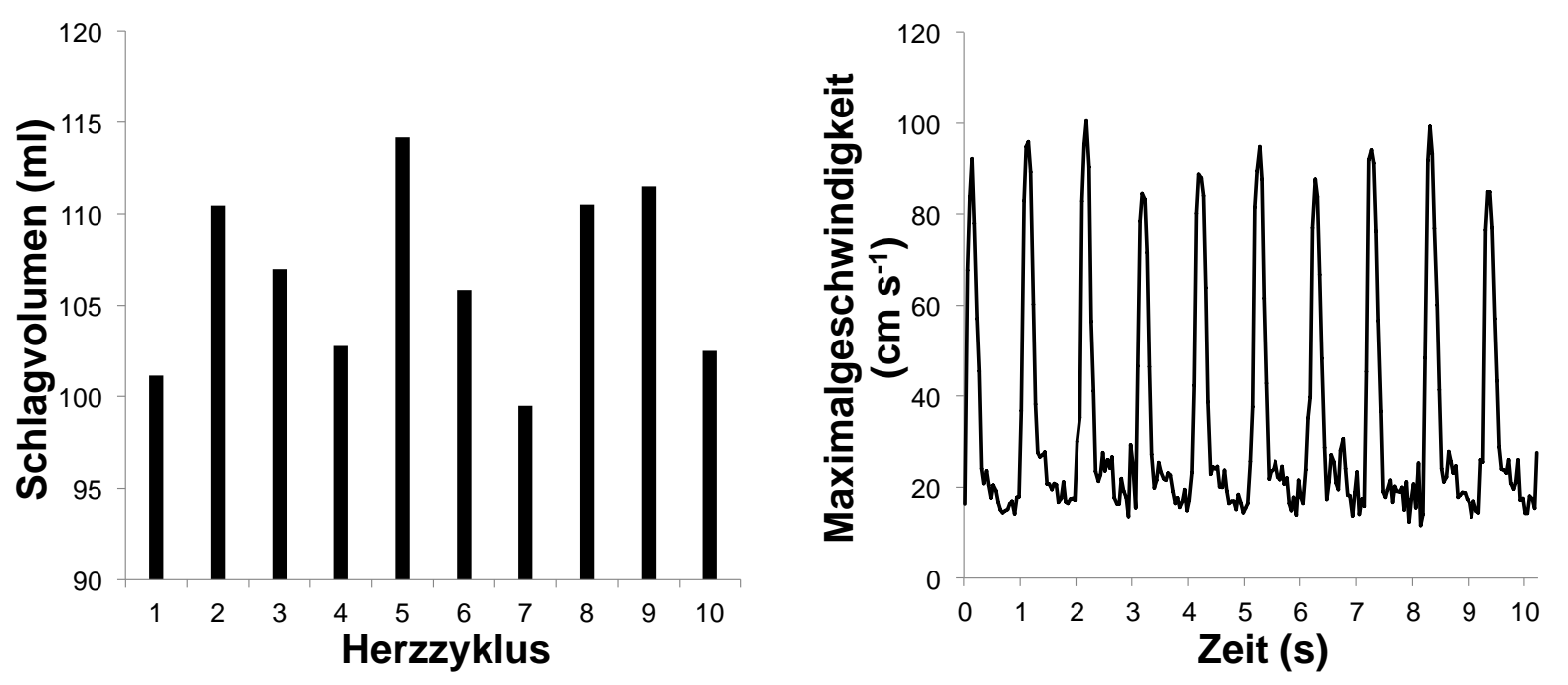

Abbildung 12: Schlagvolumen (links) und Maximalgeschwindigkeit (rechts) 10 sukzessiver Herzschläge. Werte von einem Probanden einer Echtzeit-CMRFlussmessung in der Aorta ascendens. Verwendete Sequenz: RT-CMR-Int. 
Eine derartige Darstellung von Flussvariationen war mit den konventionellen CINEPhasenkontrast-Flussmessungen durch die Interpolation mehrerer Herzschläge zu einem „synthetischen“ Herzzyklus nicht möglich.

Der Vergleich des Schlagvolumens (Flussvolumen pro Herzschlag), gemessen in der Aorta ascendens, zwischen Echtzeit-CMR- und CINE-Flussmessungen zeigte eine hohe Korrelation $(r \geq 0,98)$ mit allgemein geringen Abweichungen (Abbildung 13). Im Vergleich mit CINE-FB zeigte RT-CMR-Int systematisch signifikant höhere Werte ( $p$ $<0,001)$. Die entsprechende Bland-Altman-Analyse zeigte eine mittlere Differenz von $-5,0 \%$ mit Übereinstimmungsgrenzen von $-10,4$ bis $0,4 \%$. RT-CMR-Seq zeigte dagegen eine leichte Unterschätzung der Werte des Schlagvolumens im Vergleich zu CINE-FB $(p<0,05)$. Der korrespondierende Bland-Altman-Plot zeigte eine Abweichung von $+3,4 \%$ mit Übereinstimmungsgrenzen von $-2,9$ bis $9,7 \%$. Im Gegenteil dazu verhielten sich die ermittelten Werte des Schlagvolumens der Echtzeit-CMRFlussmessungen im Vergleich zu CINE-BH invers. Es wurde eine gute Übereinstimmung zwischen RT-CMR-Int und CINE-BH mit einer Verzerrung von nur $-0,5 \%$ (Übereinstimmungsgrenzen: $-6,8$ bis $5,8 \%)$ ohne signifikante Unterschiede $(p>0,05)$ gefunden. RT-CMR-Seq zeigte gegenüber CINE-BH dagegen eine deutliche Unterschätzung (Mittlere Differenz: $+7,9 \%$, Übereinstimmungsgrenzen: 0,8 $-15,1 \%, p<$ $0,001)$.

Der Vergleich des Schlagvolumens untereinander zwischen CINE-Flussmessungen und Echtzeit-CMR-Flussmessungen zeigte ebenfalls eine hohe Korrelation, wiederum mit systematisch geringen Abweichungen (Abbildung 14). CINE-BH zeigte im Vergleich zu CINE-FB eine signifikante Überschätzung (mittlere Differenz: $-4,5 \%$, Übereinstimmungsgrenzen: $-8,7$ bis $-0,3 \%, p<0,001$ ), während RT-CMR-Seq im Vergleich zu RT-CMR-Int eine systematische Unterschätzung des Schlagvolumens zeigte (mittlere Differenz: $+8,4 \%$, Übereinstimmungsgrenzen: 3,9 bis $19,9 \%, p<$ $0,001)$. 

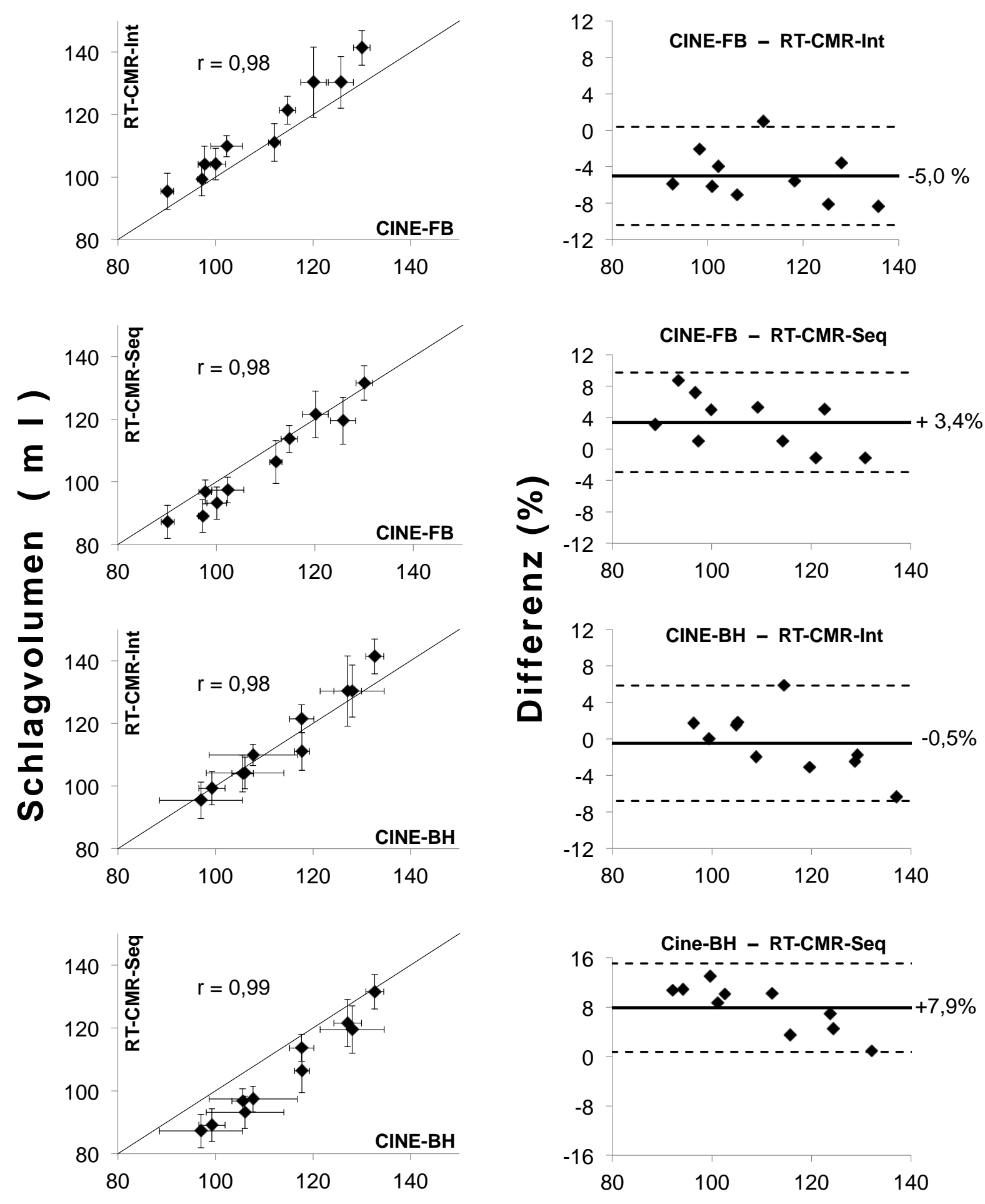

Schlagvolumen (ml)

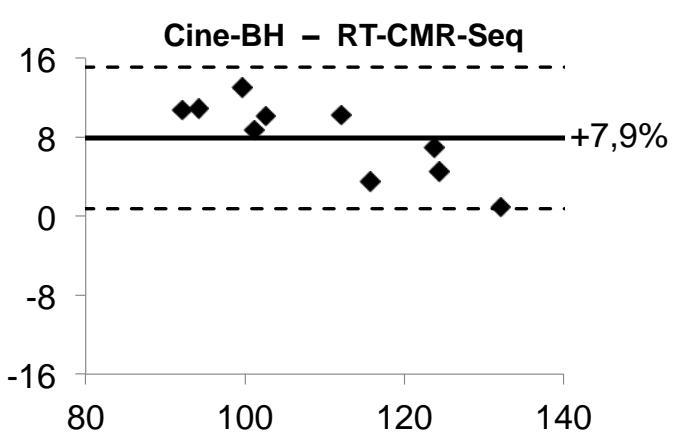

Mittel der Schlagvolumina (ml)

Abbildung 13: Links: Streudiagramm mit Winkelhalbierender. Schlagvolumina (Mittelwert \pm Standardabweichung) für Echtzeit(RT)-Flussmessungen (10 Probanden, 3 Wiederholungen, 10 Herzzyklen) vs. CINE-Flussmessungen (10 Probanden, 3 Wiederholungen, ein interpolierter Herzzyklus). Rechts: Korrespondierende BlandAltman-Plots. Durchgezogene Linie entspricht der mittleren Differenz. Gestrichelte Linien geben die Übereinstimmungsgrenzen (mittlere Differenz \pm 2 SD) an. 

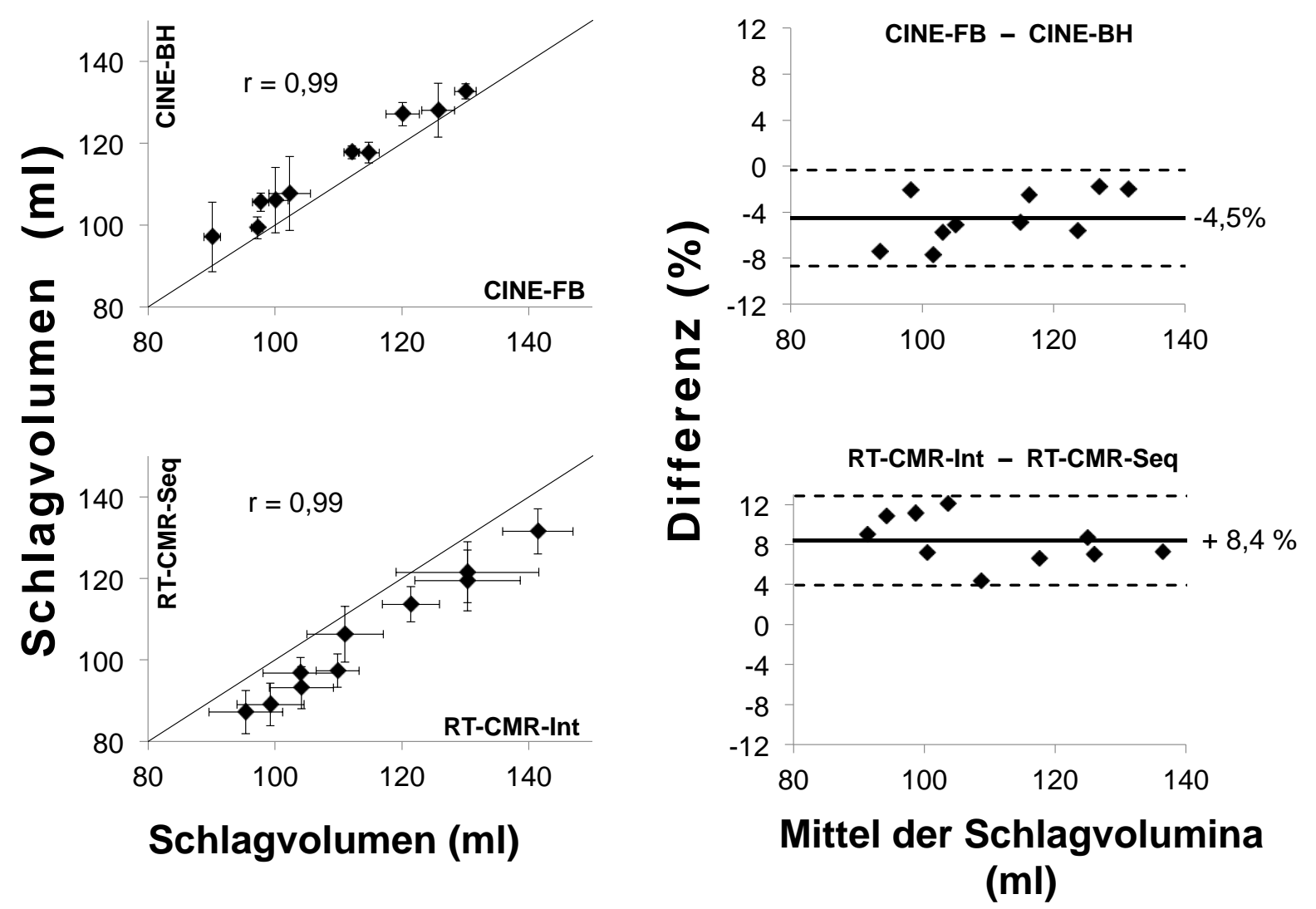

Abbildung 14: Links: Streudiagramm mit Winkelhalbierender. Oben: Schlagvolumen (Mittelwert \pm Standardabweichung) CINE-FB vs. CINE-BH (10 Probanden, 3 Wiederholungen, ein interpolierter Herzzyklus). Unten: Schlagvolumen RT-CMR-Seq vs. RT-CMR-Int (10 Probanden, 3 Wiederholungen, 10 Herzzyklen). Rechts: Korrespondierende Bland-Altman-Plots. Durchgezogene Linie entspricht der mittleren Differenz. Gestrichelte Linien geben die Übereinstimmungsgrenzen (mittlere Differenz \pm 2 $\mathrm{SD})$ an.

Eine vergleichende Analyse der Flussprofile der unterschiedlichen Sequenzen konnte zeigen, dass sich die beiden Echtzeit-Varianten bei der Messung systolischer Flussgeschwindigkeiten nicht unterschieden. Es fiel allerdings auf, dass mittels RTCMR-Seq in der frühen Diastole vermehrt negative durchschnittliche Flussgeschwindigkeiten gemessen wurden, während diese kurzen Rückflüsse bei Verwendung der RT-CMR-Int nicht sichtbar waren (Abbildung 15). Des Weiteren zeigte RT-CMR-Int - im Vergleich zu RT-CMR-Seq - im gesamten zeitlichen Bereich der Diastole höhere durchschnittliche Flussgeschwindigkeiten. Dies wird bei Betrachtung der Fläche unter der Kurve im Bereich der Diastole in Abbildung 15 am deutlichsten, die sich bei RT-CMR-Int größer als bei RT-CMR-Seq darstellte. 

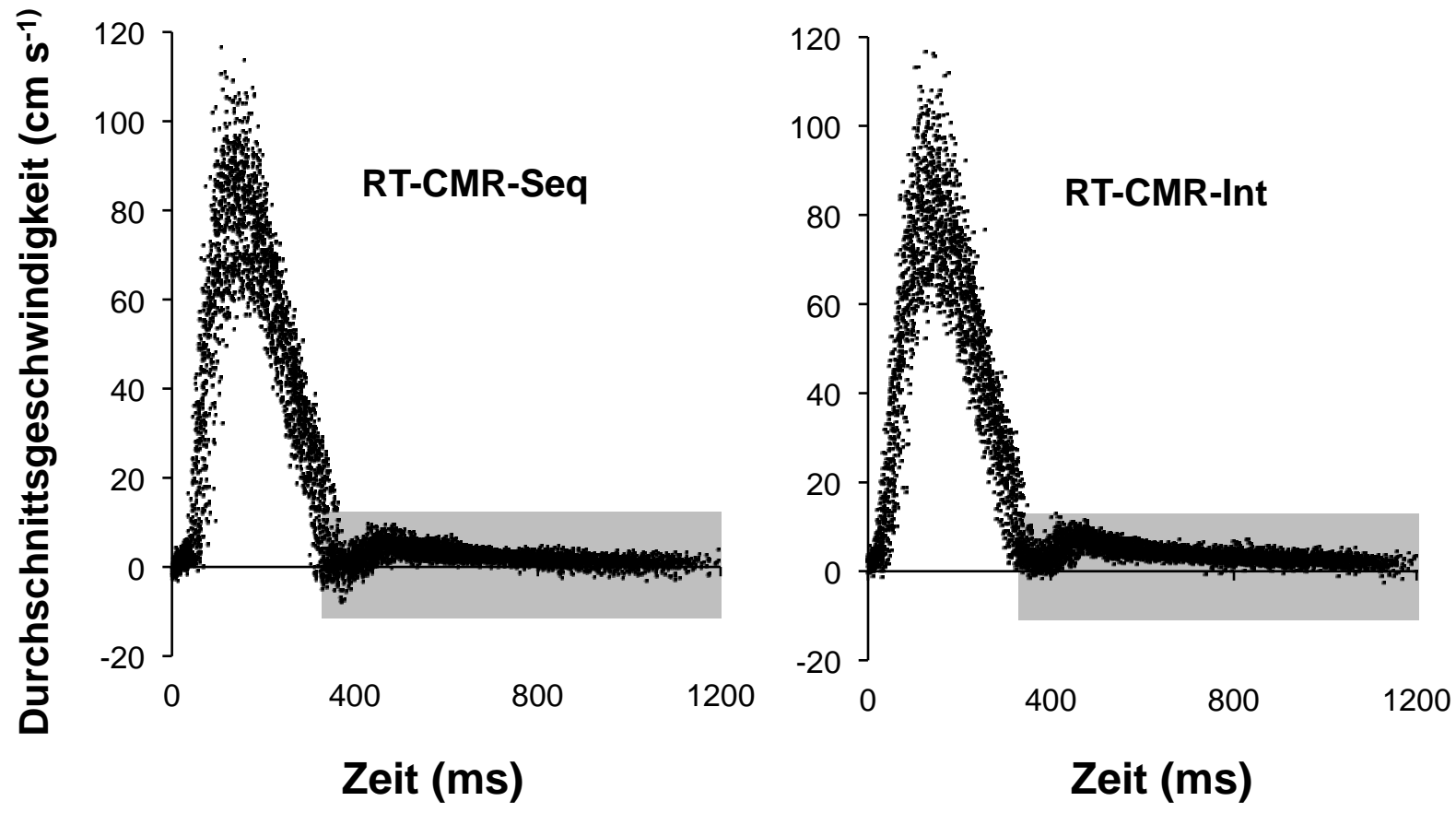

Abbildung 15: Durchschnittsgeschwindigkeit in zeitlicher Abhängigkeit von der RZacke im EKG (RT-CMR-Seq, RT-CMR-Int). Gruppenkarte aller 10 Probanden (insgesamt 300 Herzzyklen). Graue Fläche: Diastole.
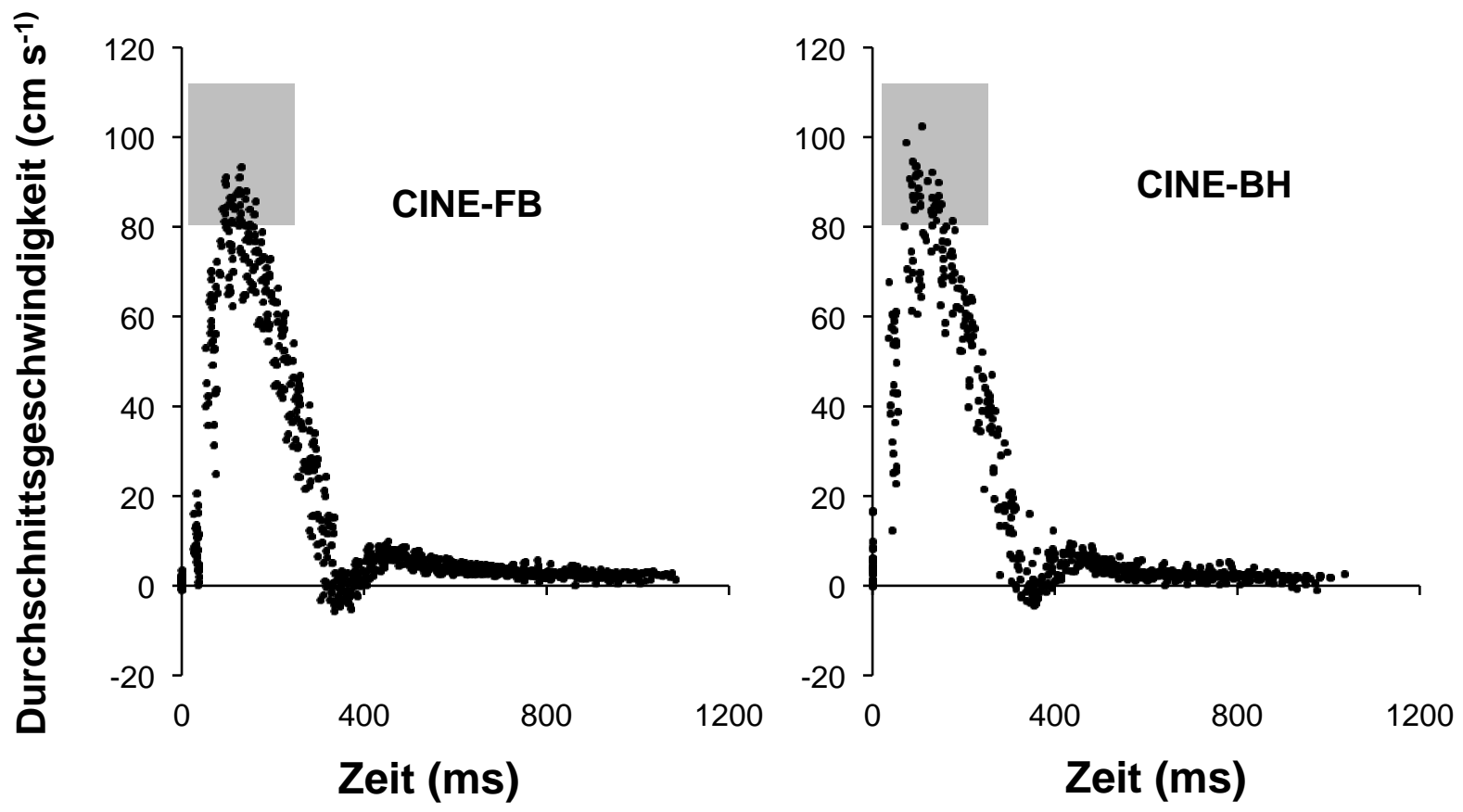

Abbildung 16: Durchschnittsgeschwindigkeit in zeitlicher Abhängigkeit von der RZacke im EKG (CINE-FB, CINE-BH). Gruppenkarte aller 10 Probanden (insgesamt 30 Herzzyklen). Graue Fläche: Maximale Systole. 
Die Flussprofile der beiden Varianten der CINE-Flussmessungen unterschieden sich in der Diastole weniger. Im zeitlichen Bereich der maximalen Systole zeigte CINE-BH im Vergleich zu CINE-FB allerdings vermehrt höhere durchschnittliche Flussgeschwindigkeiten (Abbildung 16).

Der Vergleich der Maximalgeschwindigkeiten gemessen in der Aorta ascendens zwischen Echtzeit-CMR- und CINE-Flussmessungen zeigte durchschnittlich leicht höhere maximale Flussgeschwindigkeiten bei Verwendung der Echtzeit-Verfahren (Abbildung 17). Eine hohe Korrelation konnte bei allen Vergleichen erkannt werden ( $r=$ 0,8 bis 0,91 ). Es fällt auf, dass die Standardabweichungen innerhalb der 3 Wiederholungen einer MRT-Sequenz höher sind, als bei dem Vergleich der Schlagvolumina. Die Höhe der Standardabweichungen war bei den Echtzeit-CMR-Flussmessungen (RT-CMR-Int, RT-CMR-Seq) größer als bei den CINE-Phasenkontrast Flussmessungen. Dabei muss beachtet werden, dass die Echtzeit-CMR-Flussmessungen Mittelwerte \pm Standardabweichung aus 30 Herzzyklen beinhalten, während CINEPhasenkontrast-Flussmessungen Mittelwerte \pm Standardabweichung aus nur 3 interpolierten Herzzyklen umfassen. Die korrespondierenden Bland-Altman-Analysen zeigten eine leichte Überschätzung der Echtzeit-CMR-Flussmessungen im Vergleich zu den konventionellen CINE-Flussmessungen, wobei diese Abweichung nicht signifikant waren $(p>0,05)$. Im Vergleich mit CINE-FB zeigten die Echtzeit-CMRFlussmessungen Abweichungen von $-4,6 \%$ (Übereinstimmungsgrenzen: $-21,4$ bis 12,2 \%, RT-CMR-Int) bzw. -4,9\% (Übereinstimmungsgrenzen: $-21,1$ bis $11,3 \%$, RTCMR-Seq). Die entsprechenden Streudiagramme zeigen, in welchen Bereichen die Überschätzung der Maximalgeschwindigkeit der Echtzeit-CMR-Flussmessungen zu finden war. Dabei fällt auf, dass CINE-FB und Echtzeit-CMR-Flussmessungen bis zu Werten von ca. $120 \mathrm{~cm} \mathrm{~s}^{-1}$ ähnliche Maximalgeschwindigkeiten gemessen haben. Für Maximalgeschwindigkeiten über $120 \mathrm{~cm} \mathrm{~s} \mathrm{~s}^{-1}$ haben beide Echtzeit-CMRFlussmessungen dagegen vermehrt höhere Werte erzielt. Die Abweichungen zwischen Echtzeit-CMR-Flussmessungen und CINE-BH fielen gering aus. Im Vergleich zeigte sich eine Abweichung von -1,3\% (Übereinstimmungsgrenzen: $-21,4$ bis $18,7 \%$, RT-CMR-Int) bzw. -1,6\% (Übereinstimmungsgrenzen: -19,7 bis 16,5\%, RT-CMRSeq). 

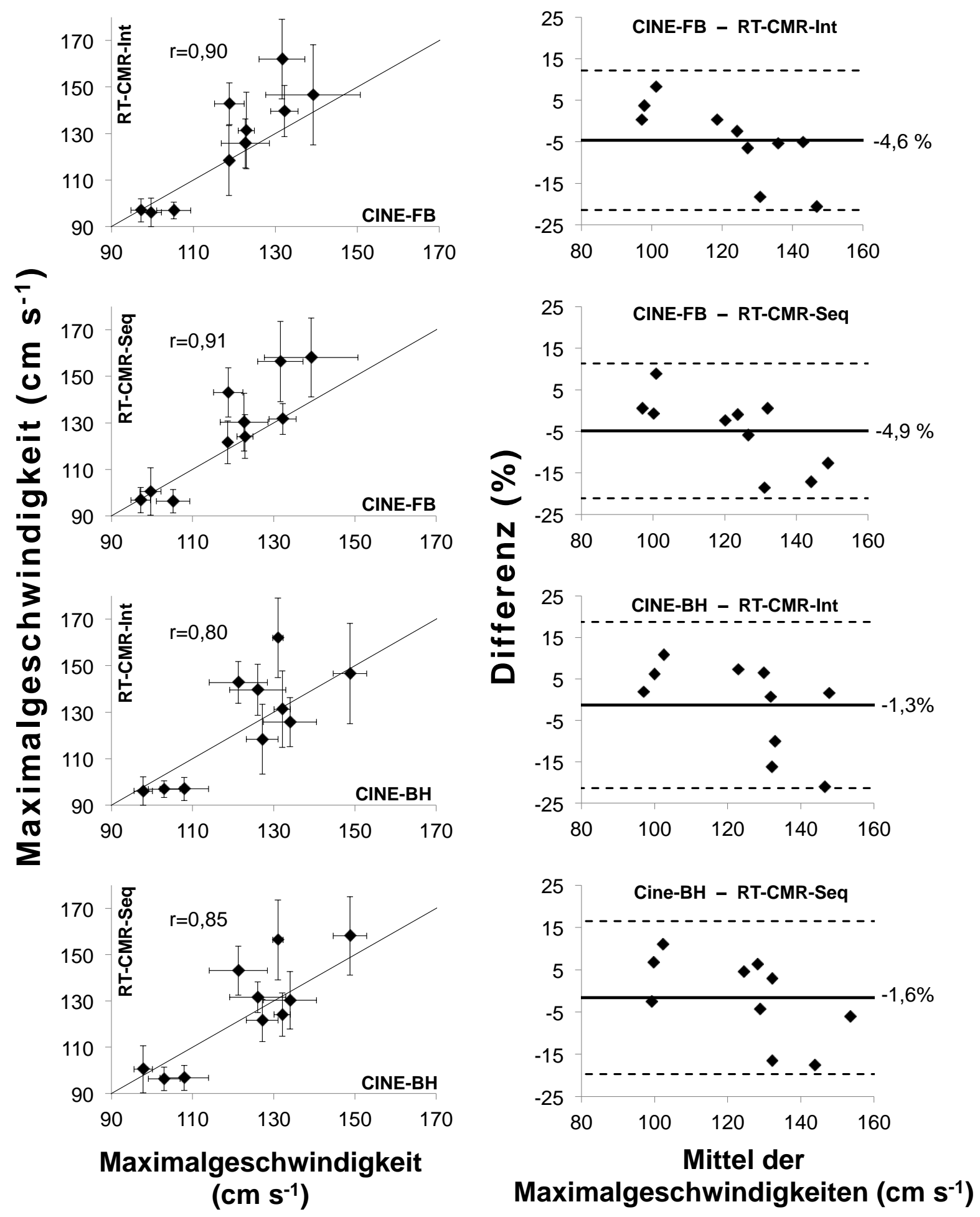

Abbildung 17: Links: Streudiagramm mit Winkelhalbierender. Maximalgeschwindigkeit (Mittelwert \pm Standardabweichung) für Echtzeit(RT)-Flussmessungen (10 Probanden, 3 Wiederholungen, 10 Herzzyklen) vs. CINE-Flussmessungen (10 Probanden, 3 Wiederholungen, ein interpolierter Herzzyklus). Rechts: Korrespondierende Bland-Altman-Plots. Durchgezogene Linie entspricht der mittleren Differenz. Gestrichelte Linien geben die Übereinstimmungsgrenzen (mittlere Differenz \pm 2 SD) an. 
Der Vergleich der Maximalgeschwindigkeiten untereinander zwischen CINE- und Echtzeit-CMR-Flussmessungen zeigte eine gute Übereinstimmung ohne signifikante Abweichungen (Abbildung 18). Sowohl CINE- als auch Echtzeit-Flussmessungen zeigten untereinander eine hohe Korrelation ( $r=0,91$ bis 0,97). CINE-FB erzielte im Vergleich zu CINE-BH im Durchschnitt 1,5\% geringere Maximalgeschwindigkeiten (Übereinstimmungsgrenzen: $-13,1$ bis 10,2 \%). Die Robustheit der technischen Abwandlungen der Echtzeit-CMR-Flussmessungen untereinander wurde bestätigt. Es konnte eine hohe Korrelation $(r=0,97)$ ohne signifikante Unterschiede $(p<0,05)$ bei einer mittleren Differenz von lediglich - $0,3 \%$ (Übereinstimmungsgrenzen: von $-8,5$ bis $7,9 \%)$ festgestellt werden.

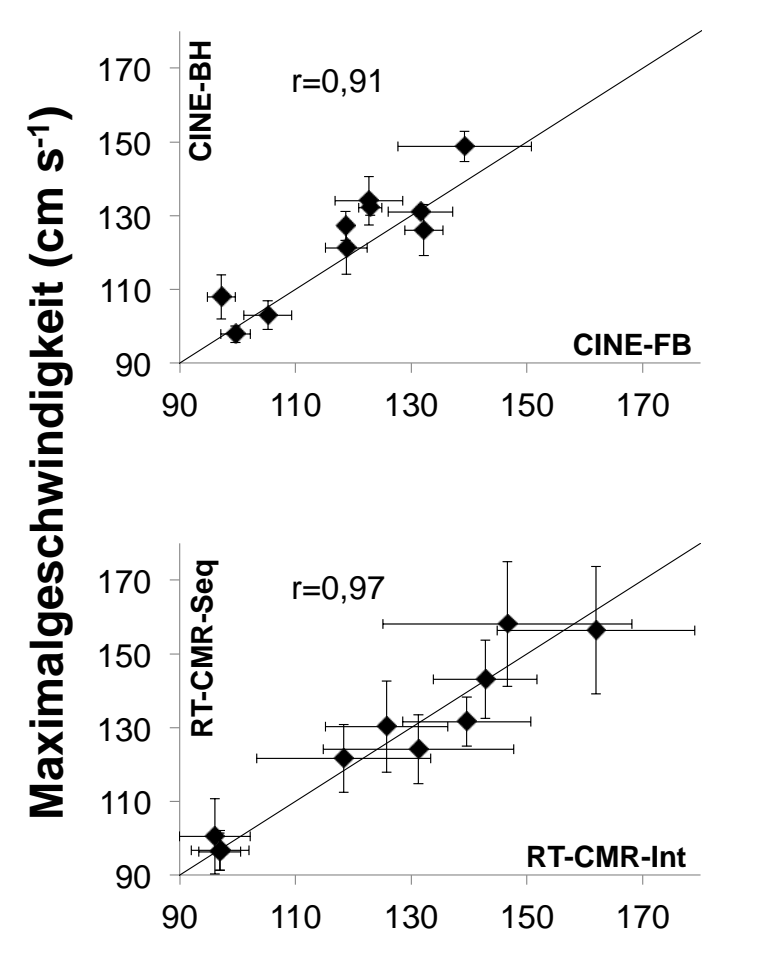

Maximalgeschwindigkeit $\left(\mathrm{cm} \mathrm{s}^{-1}\right)$

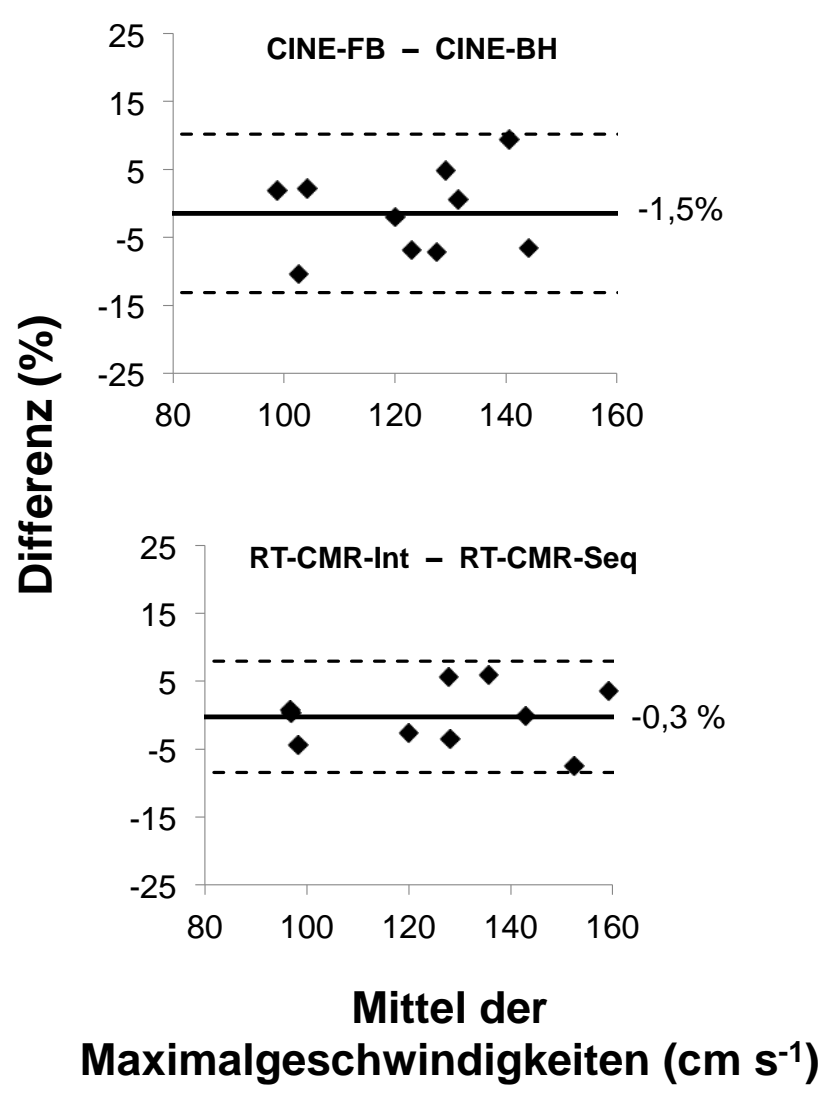

Abbildung 18: Links: Streudiagramm mit Winkelhalbierender. Oben: Maximalgeschwindigkeit (Mittelwert \pm Standardabweichung) CINE-FB vs. CINE-BH (10 Probanden, 3 Wiederholungen, ein interpolierter Herzzyklus). Unten: Maximalgeschwindigkeit RT-CMR-Seq vs. RT-CMR-Int (10 Probanden, 3 Wiederholungen, 10 Herzzyklen). Rechts: Korrespondierende Bland-Altman-Plots. Durchgezogene Linie entspricht der mittleren Differenz. Gestrichelte Linien geben die Übereinstimmungsgrenzen (mittlere Differenz \pm 2 SD) an. 


\subsubsection{Vergleich der Auswertbarkeit}

Die Anzahl rekonstruierter Phasen unterschied sich zwischen den verwendeten Sequenzen. Bei der CINE-FB wurden 30 Phasen und bei der CINE-BH 20 Phasen rekonstruiert. Die Echtzeit-Sequenzen wurden für 15 Sekunden akquiriert. Bei einer zeitlichen Auflösung von $40 \mathrm{~ms}$ resultierten daraus entsprechend 375 Phasen. Die ersten 10 Herzzyklen einer Echtzeit-Messung wurden in die Auswertung eingeschlossen. Im Durchschnitt wurden pro Echtzeit-Messung 238 (RT-CMR-Int) bzw. 235 (RT-CMR-Seq) Phasen ausgewertet. Der prozentuale Anteil korrekturbedürttiger Phasen war in den Echtzeit-Messungen geringer als in den CINE-Flussmessungen. Die absolute Anzahl korrekturbedürftiger Phasen war bei den Echtzeit-Sequenzen aufgrund der größeren Datenmenge entsprechend höher, was sich in der GesamtKorrekturzeit widerspiegelte. Die Korrekturzeit eines einzelnen Herzzyklus zeigte sich in den Echtzeit-CMR-Methoden geringer als die Auswertung eines interpolierten Herzzyklus der CINE-Flussmessungen (Tabelle 4).

Tabelle 4: Anteil manuell korrigierter Phasen und Korrekturzeit. Angaben ergeben sich als Mittelwert \pm Standardabweichung von 10 Probanden und 3 Wiederholungen. Die Korrekturzeiten sind für die Echtzeit(RT)-CMR-Flussmessungen zusätzlich für einen einzelnen Herzzyklus berechnet.

Anteil korrigierter Phasen (\%) Korrekturzeit (min:sec)

CINE-FB

$38,1 \pm 14,1$

$01: 25 \pm 00: 43$

CINE-BH

$47,3 \pm 21,1$

$01: 24 \pm 01: 18$

RT-CMR-Int

$27,4 \pm 10,5$

09:41 $\pm 04: 57^{*}$

pro Herzschlag

00:58 $\pm 00: 30$

RT-CMR-Seq

$25,6 \pm 9,3$

09:22 $\pm 05: 04$ *

pro Herzschlag

00:56 \pm 00:30

* Korrekturzeit für 10 sukzessive Herzzyklen 
Die Tabelle 5 vergleicht die ermittelten Werte des Schlagvolumens und der Maximalgeschwindigkeit nach voll-automatischer Segmentationsanalyse und nach manueller Konturkorrektur. Die manuelle Konturkorrektur hatte allgemein nur einen geringen Einfluss auf die Parameter Schlagvolumen und Maximalgeschwindigkeit. Die Werte waren bei allen vier MRT-Sequenzen nach der manuellen Konturkorrektur konsequent leicht niedriger. Im zweiseitigen, gepaarten t-test stellten sich nur der minimale Unterschied im Schlagvolumen von durchschnittlich 0,3 $\mathrm{ml}$ (CINE-FB) und der Unterschied der Maximalgeschwindigkeit von durchschnittlich $3,7 \mathrm{~cm} \mathrm{~s}^{-1}$ (RTCMR-Seq) als signifikant $(p<0,05)$ heraus.

Tabelle 5: Schlagvolumen $(\mathrm{ml})$ und Maximalgeschwindigkeit $\left(\mathrm{cm} \mathrm{s}^{-1}\right)$ in der Aorta ascendens (Mittelwert \pm Standardabweichung). Werte resultieren für CINEFlussmessungen aus 10 Probanden und 3 Wiederholungen mit einem interpolierten Herzzyklus, für Echtzeit(RT)-CMR-Flussmessungen aus 10 Probanden und 3 Wiederholungen mit 10 Herzzyklen.

\begin{tabular}{ccc}
\hline voll-automatische & nach manueller & t-test \\
Analyse & Konturkorrektur & $(p$ Wert $)$
\end{tabular}

\section{CINE-FB}

Schlagvolumen

Maximalgeschwindigkeit

$$
109,3 \pm 2,3
$$

$109,0 \pm 1,9$

$0,01^{*}$

$121,3 \pm 8,2$

$118,8 \pm 4,2$

0,34

\section{CINE-BH}

Schlagvolumen

Maximalgeschwindigkeit

\section{RT-CMR-Int}

Schlagvolumen

Maximalgeschwindigkeit

\section{RT-CMR-Seq}

Schlagvolumen

Maximalgeschwindigkeit
$114,4 \pm 5,7$

$124,6 \pm 6,6$
$113,9 \pm 4,6$

0,06

$122,9 \pm 4,4$

0,30

$\begin{array}{lccc}\text { Schlagvolumen } & 106,2 \pm 5,8 & 105,6 \pm 5,5 & 0,15 \\ \text { Maximalgeschwindigkeit } & 130,8 \pm 15,7 & 125,9 \pm 10,3 & 0,07\end{array}$

${ }^{*}$ signifikant $(p<0,05)$ 


\subsubsection{Vergleich mit der Doppler-Echokardiographie}

Bei allen Probanden konnte die Doppler-echokardiographische Flussmessung erfolgreich durchgeführt werden. Bei 9 Probanden wurden alle Messungen im apikalen Fünf-Kammerblick für die Auswertung verwendet. Bei einem Probanden waren die Schallbedingungen im apikalen Fünf-Kammerblick und in der parasternalen langen Achse stark eingeschränkt, sodass hier die Doppler-Messungen aus einem suprasternalen Schallfenster in der statistischen Analyse verwendet wurden.

Der Vergleich der Maximalgeschwindigkeit zwischen MRT-Flussmessungen und Doppler-Messungen zeigte mit Korrelationskoeffizienten zwischen $r=0,43$ und $r=$ 0,81 einen schwächeren linearen Zusammenhang als bei dem Vergleich der Maximalgeschwindigkeit innerhalb der MRT-Flussmessungen (Abbildung 19). Es fällt auf, dass die Standardabweichung bei den CINE-Flussmessungen am geringsten ausgefallen ist. Hierbei ist zu beachten, dass diese lediglich Werte aus 3 interpolierten Herzzyklen in der Darstellung beinhalten. Die Standardabweichungen der Echtzeit-CMR- und der Doppler-Messungen waren höher. Die entsprechenden Angaben beinhalten hierbei Werte von 30 (RT-CMR) bzw. 9 Herzzyklen (Doppler).

Die Bland-Altman-Analyse zeigte geringe Abweichungen zwischen den gemessenen Maximalgeschwindigkeiten aus den MRT- und Doppler-Flussmessungen. Alle MRTFlussmessungen zeigten durchschnittlich leicht höhere Werte als die DopplerMessungen. Für CINE-FB zeigte sich im Vergleich zu den Ergebnissen aus der Doppler-Echokardiographie eine Abweichung von -1,1\% (Übereinstimmungsgrenzen: $-27,0$ bis $24,9 \%, p>0,05$ ). CINE-BH zeigte eine Abweichung von $-2,6 \%$, wobei hier die beste Korrelation mit $r=0,81$ vorlag und die Übereinstimmungsgrenzen mit -22,1 bis $17,0 \%$ am geringsten ausgefallen sind $(p>0,05)$. Die Echtzeit-CMRFlussmessungen zeigten im Vergleich Abweichungen von $-3,8 \%$ (RT-CMR-Int, $r=$ $0,43, p>0,05$ ) und $-4,1 \%$ (RT-CMR-Seq, $r=0,44, p>0,05$ ) mit größeren Übereinstimmungsgrenzen, die von $-38,9 \%$ bis $31,3 \%$ (RT-CMR-Int) und $-39,2 \%-31,0 \%$ (RT-CMR-Seq) reichten. 

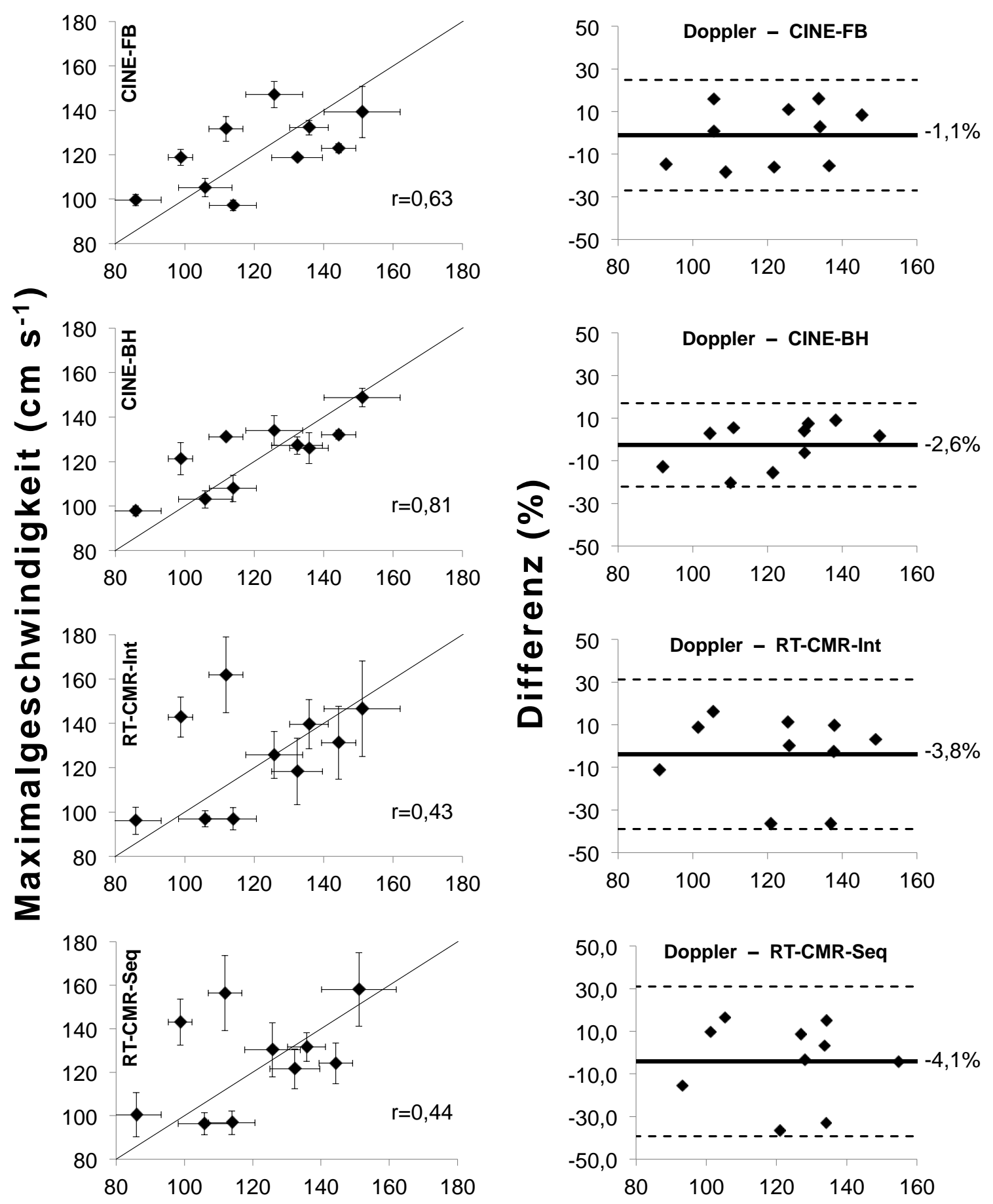

Maximalgeschwindigkeit $\left(\mathrm{cm} \mathrm{s}^{-1}\right)$
Doppler

Mittel der Maximalgeschwindigkeiten ( $\left.\mathrm{cm} \mathrm{s}^{-1}\right)$

Abbildung 19: Links: Streudiagramm mit Winkelhalbierender. Maximalgeschwindigkeit (Mittelwert \pm Standardabweichung) für Echtzeit(RT)-CMR-Flussmessungen (10 Probanden, 3 Wiederholungen, 10 Herzzyklen) und CINE-Flussmessungen (10 Probanden, 3 Wiederholungen) vs. Ultraschall Doppler-Messungen (10 Probanden, 3 Wiederholungen, 3 Herzzyklen). Rechts: Korrespondierende Bland-Altman-Plots. Durchgezogene Linie entspricht der mittleren Differenz. Gestrichelte Linien geben die Übereinstimmungsgrenzen (mittlere Differenz \pm 2 SD) an. 


\subsection{Anwendung der Echtzeit-CMR-Flussmessung mit dem Valsalva-Manöver als physiologischem Stressor}

Bei allen 20 Probanden konnten die Echtzeit-CMR-Flussmessungen mit gleichzeitiger intraoraler Druckmessung - sowohl unter Ruhebedingungen als auch unter dem Valsalva-Manöver - erfolgreich durchgeführt werden. Die im Folgenden beschriebenen Ergebnisse wurden bereits unter Kowallick et al. 2014 b publiziert.

Es wurden insgesamt 60 Messungen unter dem Valsalva-Manöver bei 20 Probanden (3 Wiederholungen) durchgeführt. Ein Proband erreichte den Zieldruck von $40 \mathrm{mmHg}$

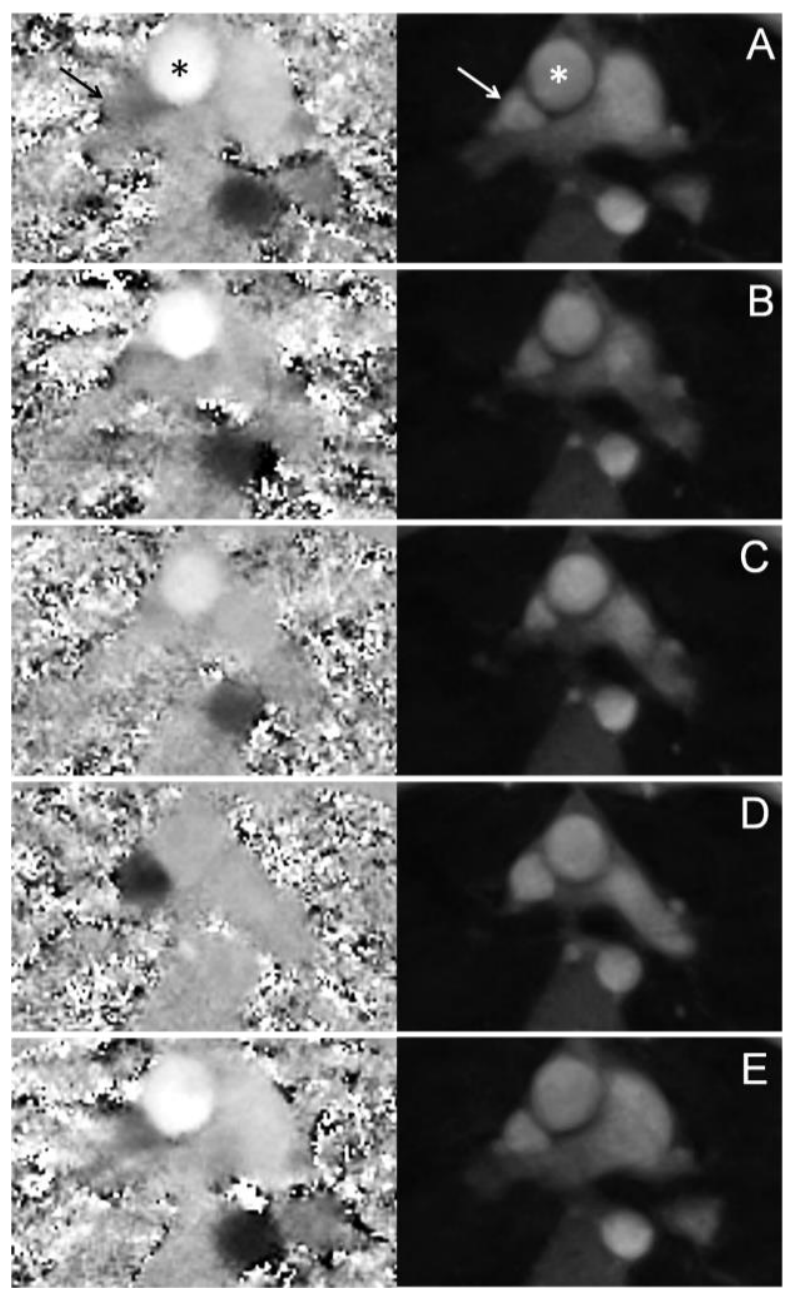

Abbildung 20: Echtzeit-CMR-Flussmessung in den unterschiedlichen Phasen eines Valsalva-Manövers. Linke Spalte: Phasenbild. Rechte Spalte: entsprechendes Magnitudenbild. A: normale Atmung, B: frühe Anspannung, C: späte Anspannung, D: frühe Erholung, E: späte Erholung. * Aorta ascendes, Pfeil: Vena cava superior. in keiner der 3 Wiederholungen. In 4 weiteren Einzelmessungen wurde der Zieldruck nicht über die erforderliche Zeit von 10 Sekunden aufrechterhalten. Diese 7 Messungen wurden von der statistischen Analyse ausgeschlossen. Zusätzlich konnte das EKG-Signal bei einer Messung während der späten Anspannungsphase und bei 5 Messungen während der frühen Erholungsphase nicht adäquat abgeleitet werden. Demnach wurden die Werte von insgesamt 19 Probanden und 53 Messungen (normale Atmung, frühe Anspannung, späte Erholung) bzw. 52 Messungen (späte Erholung) und 48 Messungen (frühe Erholung) in die statistische Analyse eingeschlossen. Eine Echtzeit-MRTMessung über 40 Sekunden resultierte in 994 Phasen bzw. 1988 rekonstruierten Bildern (Magnituden- und Phasenbild). Es wurden demnach ca. 60.000 Phasen mit 120.000 Bildern 
analysiert. Die Abbildung 20 zeigt eine Abfolge von Magnituden- und Phasenbildern einer Echtzeit-CMR-Flussmessung unter dem Valsalva-Manöver in den 5 unterschiedlichen Phasen. Darauf ist erkennbar, dass der erhöhte intrathorakale Druck zu einer Kompression der großen thorakalen Gefäße führte. Dieser Effekt zeigte sich am deutlichsten in der SVC während der späten Anspannungsphase. In der frühen Erholungsphase zeigte die SVC eine Zunahme des Gefäßlumens und eine Änderung der Form von dreieckig bis nahezu rund.

\section{Hämodynamik unter dem Valsalva-Manöver}

Die hämodynamischen Variationen konnten in aufeinanderfolgenden Herzzyklen zuverlässig in Echtzeit dargestellt werden. Die Abbildung 21 zeigt eine Übersicht der Änderungen des Flussvolumens pro Herzschlag, der Gefäßquerschnittsfläche und der Herzfrequenz bei einem Probanden in den unterschiedlichen Phasen eines Valsalva-Manövers. Die Tabelle 6 zeigt die Werte dieser Parameter (Mittelwert \pm SD und in prozentualem Verhältnis zu Werten in normaler Atmung) gemittelt über alle Messungen der 19 Probanden.

Während des Valsalva-Manövers wurde ein signifikanter Abfall des Blutflussvolumens pro Herzschlag in der AAo (normale Atmung: $87 \pm 16 \mathrm{ml}$, späte Anspannung: $37 \pm 13 \mathrm{ml}, \mathrm{p}<0,001$ ) und SVC (normale Atmung: $28 \pm 8 \mathrm{ml}$, späte Anspannung: 10 $\pm 6 \mathrm{ml}, \mathrm{p}<0,001$ ) beobachtet. Zu Beginn eines Valsalva-Manövers blieb der Blutfluss in der AAo unverändert (frühe Anspannung: $91 \pm 16 \mathrm{ml}, \mathrm{p}>0,05$ ) und nahm anschließend kontinuierlich ab, während er in der SVC abrupt sistierte (frühe Anspannung: $-3 \pm 6 \mathrm{ml}, p<0,001$ ) und stetig auf einem niedrigen Level blieb. Sobald die Probanden aufhörten zu pressen, kam es in der SVC zu einem Überschuss des Flussvolumens (frühe Erholung: $52 \pm 16 \mathrm{ml}, \mathrm{p}<0,001$ ), während der Blutfluss in der $A A o$ in dieser Phase weiterhin reduziert blieb (frühe Erholung: $33 \pm 12 \mathrm{ml}, \mathrm{p}<0,001$ ) und sich erst 3 bis 4 Herzzyklen später erholte (Abbildung 21). Die Herzfrequenz erhöhte sich im Verlauf des Pressversuchs kontinuierlich (frühe Anspannung: $75 \pm 8$ bpm, späte Anspannung: $98 \pm 16$ bpm, $p<0,001$ ), blieb direkt nach dem ValsalvaManöver erhöht (frühe Anspannung: $99 \pm 15$ bpm, $p<0,001$ ) und zeigte anschlieBend im Vergleich zu Werten vor dem Valsalva-Manöver signifikant niedrigere Werte (späte Erholung: $68 \pm 9$ bpm, $p<0,001$ ). Wie zuvor in Abbildung 20 visuell gezeigt, 
wurden die großen thorakalen Gefäße durch den erhöhten intrathorakalen Druck komprimiert. Die Gefäßquerschnittsfläche der AAo wurde durchschnittlich um $17 \%$ (normale Atmung: $728 \pm 141 \mathrm{~mm}^{2}$, späte Anspannung: $601 \pm 133 \mathrm{~mm}^{2}, \mathrm{p}<0,001$ ), die der SVC durchschnittlich um $35 \%$ reduziert (normale Atmung: $296 \pm 6 \mathrm{~mm}^{2}$, späte Anspannung: $188 \pm 58 \mathrm{~mm}^{2}, \mathrm{p}<0,001$ ).

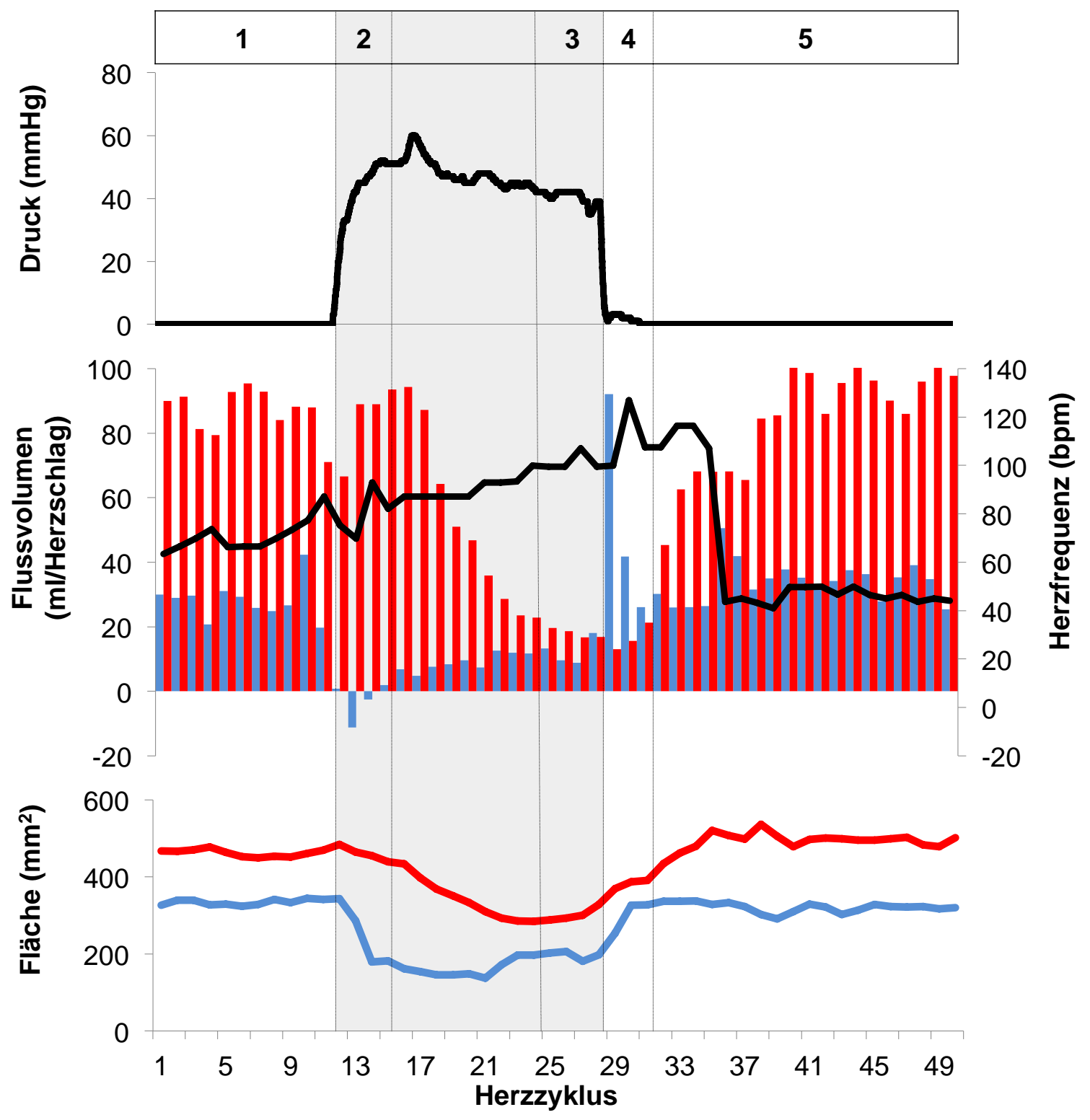

Abbildung 21: Hämodynamische Reaktion unter dem Valsalva-Manöver in der Aorta ascendens (rot) und Vena cava superior (blau) bei einem 24-jährigen Probanden. Graue Zone: 10 Sekunden Valsalva-Manöver. Die Zahlen in dem Balken erläutern die Unterteilung der Messungen in 5 Phasen (1: normale Atmung, 2: frühe Anspannung, 3: späte Anspannung, 4: frühe Erholung, 5: späte Erholung). 
Tabelle 6: Hämodynamische Variationen unter dem Valsalva-Manöver in der Vena cava superior und Aorta ascendens.

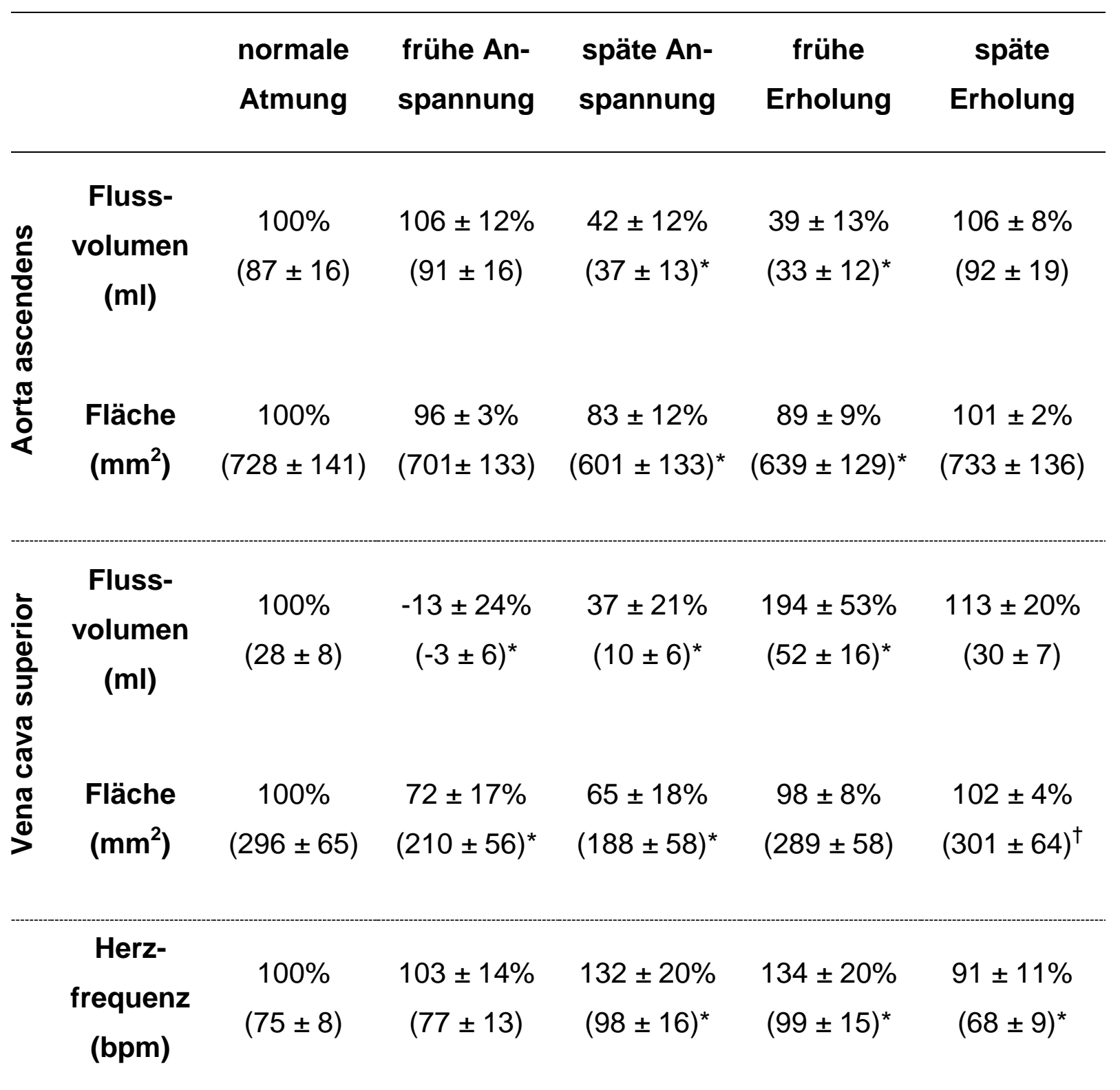

Werte sind angegeben in prozentualem Verhältnis zu den Werten während der normalen Atmung und als Mittelwert \pm SD (19 Probanden). Das Signifikanzniveau ist relativ zu den Werten während der normalen Atmung angegeben:

${ }^{*} p<0,001$

$+p<0,05$ 


\section{Diskussion}

\subsection{Vergleich von Echtzeit-CMR-Flussmessung und CINE-Flussmessung}

Ziel der hier vorliegenden Arbeit war die Evaluation eines neu entwickelten EchtzeitVerfahrens zur quantitativen Flussmessung in der MRT. Das Verfahren wurde bezüglich des Auftretens von Phasen-Offset-Fehlern, der Erfassung hämodynamischer Parameter und der Auswertbarkeit der erhobenen Datensätze überprüft. Als Referenzstandard dienten klinisch etablierte CINE-Flussmessungen bzw. die DopplerEchokardiographie. Ein Vorteil des Echtzeit-Verfahrens besteht darin beat-to-beatFlussvariationen darzustellen. Diese Möglichkeit wurde genutzt, um die hämodynamischen Auswirkungen eines physiologischen Stressors - wie dem ValsalvaManöver - mit Hilfe der neu entwickelten MRT-Technik in Echtzeit darzustellen.

Die Validierung der als Referenzstandard verwendeten CINE-Flussmessungen zur Bestimmung von Flussvolumina, Klappenstenosen, Klappeninsuffizienzen und Shuntvolumina ist zuvor vielfach erfolgt (Beerbaum et al. 2008; Kozerke et al. 2001; Makowski et al. 2011). Dennoch beinhalten die CINE-Flussmessungen für den klinischen Einsatz bedeutsame Limitationen. Die reale zeitliche Auflösung der interpolierten Daten ist deutlich schlechter als vergleichbare Verfahren wie z. B. die DopplerEchokardiographie. Die Technik der CINE-Flussmessung setzt voraus, dass der zu messende Blutfluss streng periodisch verläuft. Diese Voraussetzung ist bei Patienten mit Arrhythmien nicht gegeben, was zu größeren Abweichungen der erfassten Flussparameter bis hin zur Unbrauchbarkeit einer kardialen MRT-Untersuchung führen kann (Lardo et al. 2005; McVeigh et al. 1998). Diese Limitationen der konventionellen CINE-Flussmessung für den klinischen Einsatz haben zur Entwicklung einer ganzen Reihe von MRT-Verfahren geführt, die eine Datenaufnahme in Echtzeit oder näherungsweise Echtzeit erlauben (Hansen et al. 2012; Keenan and Pennell 2007). Die meisten dieser Verfahren erreichen die beschleunigte Aufnahme durch eine gezielte Unterabtastung der räumlichen Daten, die zu einem Verlust an örtlicher Auflösung führt. Die kardiale MRT-Flussmessung stellt in erste Linie eine quantitative Auswertung dar, in der morphologische Details der Bilder nur eine untergeordnete Rolle spielen. Dennoch ist auch hierbei eine adäquate Ortsauflösung Voraussetzung, um eine gute Abgrenzbarkeit der Gefäßwände zu gewährleisten, die für die Funktions- 
analyse unverzichtbar ist. Eine der bekanntesten technischen Varianten einer schnellen Flussmessung ist die Flussmessung auf Grundlage der echo-planaren (EPI) Bildgebung (Debatin et al. 1995 b; Eichenberger et al. 1995; Guilfoyle et al. 1991). Ihr Nachteil ist die hohe Artefaktanfälligkeit, verbunden mit einem niedrigen Signal-zuRauschverhältnis, was zu Ungenauigkeiten bei der Flussquantifizierung führen kann (Walker et al. 1993). Andere Methoden der Rekonstruktion mittels paralleler Bildgebung, wie die k-t-Blast Technik, ermöglichen ebenfalls eine rasche Flussmessung (Thunberg et al. 2012). Wiederum stellen auch hier die nachteilige Ortsauflösung und die Artefaktanfälligkeit begrenzende Faktoren dar. Ein weiterer Grund für die fehlende Etablierung in der klinischen Routine ist darin zu sehen, dass diese Techniken vor der eigentlichen Flussmessung auf einen Referenzscan angewiesen sind. Dieser ist notwendig um die Empfindlichkeitsprofile aller Spulenelemente vor der Datenakquisition zu ermitteln (Pruessmann et al. 1999). Die zeitliche Ersparnis durch eine schnellere Datenakquisition hebt sich dabei durch die Länge der vorbereitenden Messung zum Teil wieder auf. Des Weiteren setzt die Technik voraus, dass zwischen Referenzscan und Datenakquisition keine Verschiebung der Spulenelemente stattfindet. Dies bedeutet im Umkehrschluss, dass zwischen Referenzscan und Datenakquisition keine Verschiebung der Anatomie, beispielsweise durch Muskelkontraktionen oder Bewegungen des Zwerchfells, stattfinden darf. Diese Voraussetzung ist in der kardiovaskulären Bildgebung selten gegeben, sodass eine fehlerhafte Registrierung der Spulenprofile unweigerlich zu Rekonstruktionsartefakten führt (Niendorf and Sodickson 2006). Das in dieser Studie verwendete Echtzeit-Verfahren ist dagegen autokalibrierend, sodass ohne Referenzscan sofort mit der Flussmessung begonnen werden kann und oben genannte Fehler ausgeschlossen werden können.

Ein weiterer Vorteil der in dieser Studie eingesetzten Echtzeit-CMR-Flussmessung ist die Kombination von hoher zeitlicher und räumlicher Auflösung. Diese technischen Eigenschaften werden mit den oben beschriebenen Echtzeit-Verfahren bislang nicht erreicht. Der Vergleich der Bildqualität der Echtzeit-CMR-Flussmessung mit den $\mathrm{Cl}$ NE-Flussmessungen bestätigte eine hohe Bildqualität der Echtzeit-Daten. Die geringen Streifenartefakte in der frühen Systole, die auf die radiale k-Raumauslesung zurückzuführen sind, zeigten sich hauptsächlich in peripheren Bildabschnitten und führten deshalb zu keinem Qualitätsverlust im Untersuchungsfeld der Aorta ascendens. Ein weiterer Vorteil der Echtzeit-CMR-Flussmessung ist die Unabhängigkeit 
einer EKG-Synchronisation. Eine zuverlässige quantitative Bestimmung des Blutflusses kann deshalb auch bei Patienten durchgeführt werden, deren Blutfluss aufgrund von Arrhythmien nicht streng periodisch verläuft. Dies ist bei den konventionellen $\mathrm{Cl}$ NE-Verfahren nicht gegeben.

Die Echtzeit-CMR-Flussmessungen können fakultativ in freier Atmung, in Atemstillstand oder unter verschiedenen Atemmanövern durchgeführt werden. Dadurch lässt sich der Blutfluss zuverlässig und vor allem schnell bei Patienten bestimmen, die konstitutionsbedingt oder aufgrund ihrer Erkrankung nicht in der Lage sind, die Atmung über einen längeren Zeitraum anzuhalten. In der klinischen Routine wird derzeit bei entsprechenden Fällen die konventionelle CINE-Flussmessung in freier Atmung verwendet, die eine Untersuchungszeit von ca. 3,5 Minuten in Anspruch nimmt. Unter der Berücksichtigung, dass der Blutfluss bei Herzklappenvitien im klinischen Alltag in der Regel in drei Ebenen gemessen wird (unter der Klappe, auf Klappenebene, über der Klappe), nimmt eine adäquate MRT-Flussmessung teilweise bis zu 10 Minuten der Untersuchungszeit für nur eine Herzklappe in Anspruch (Cawley et al. 2009). Durch eine entsprechende Anwendung der Echtzeit-CMR-Flussmessung von beispielsweise 10 Sekunden in jeder der drei Ebenen (insgesamt 30 Sekunden) könnte die Untersuchungszeit massiv reduziert werden.

\section{Empfindlichkeit der MRT-Flussmessungen gegenüber Phasen-Offset-Fehlern}

Phasen-Offset-Fehler können bei MRT-Flussmessungen zu erheblichen Abweichungen der Flussparameter führen und gehören zu den vom Untersucher unbeeinflussbaren Fehlerquellen. Das Ausmaß an Phasen-Offset-Fehlern wurde mit Hilfe eines statischen Phantoms bestimmt. Die Ergebnisse aus den Phantommessungen haben gezeigt, dass weniger Phasen-Offset-Fehler bei den Echtzeit-CMR-Flussmessungen im Bereich der Aorta ascendens auftreten als bei konventionellen CINEFlussmessungen.

Frühere Studien haben das Auftreten von Phasen-Offset-Fehlern bei CINEPhasenkontrast-Flussmessungen mehrfach untersucht (Chernobelsky et al. 2007; Lankhaar et al. 2005; Miller et al. 2009). Aus diesen Arbeiten ergibt sich als zusammenfassende Konsequenz, dass ein Phasen-Offset-Fehler von $0,6 \mathrm{~cm} \mathrm{~s}^{-1}$ als akzep- 
tabler Grenzwert angesehen werden kann. Bei einer durchschnittlichen aortalen Gefäßquerschnittsfläche eines Erwachsenen von $7 \mathrm{~cm}^{2}$ läge der Fehler bei der Messung des Schlagvolumens entsprechend unter $5 \%$ (Gatehouse et al. 2010). Die Ergebnisse dieser Studie haben gezeigt, dass die Höhe an Phasen-Offset-Artefakten bei den verwendeten Echtzeit-CMR-Flussmessungen deutlich unter dem akzeptablen Grenzwert von $0,6 \mathrm{~cm} \mathrm{~s}^{-1}$ liegt. Das Auftreten von Phasen-Offset-Artefakten war dagegen bei den konventionellen CINE-Phasenkontrast Flussmessungen höher. Der Fehler der CINE-BH lag ebenfalls unter dem annehmbaren Grenzwert von 0,6 cm${ }^{-1}$, wohingegen CINE-FB das Limit bei einem durchschnittlichen Phasen-Offset-Fehler von $1,1 \mathrm{~cm} \mathrm{~s}^{-1}$ überschritten hat.

Eine Schwierigkeit der Erfassung von Phasen-Offset-Artefakten liegt darin, dass die interindividuelle Variabilität oftmals hoch ist (Gatehouse et al. 2012). Interessanterweise konnte gezeigt werden, dass die Standardabweichung bei Mittelung des Phasen-Offset-Fehlers über alle Probanden bei den Echtzeit-CMR-Flussmessungen deutlich geringer ist als bei den konventionellen CINE-Flussmessungen. Dies zeigt, dass Phasen-Offset-Artefakte bei den Echtzeit-CMR-Flussmessungen homogen und vor allem konsequent auf niedrigem Niveau aufgetreten sind, während die größeren Standardabweichungen bei den konventionellen CINE-Flussmessungen eine höhere interindividuelle Variabilität bestätigen.

In der vorliegenden Arbeit wurde nur der Phasen-Offset-Fehler in der Aorta ascendens bestimmt. Frühere Studien haben gezeigt, dass das Auftreten von PhasenOffset-Artefakten vor allem im Bereich des rechtsventrikulären Ausflusstraktes von Bedeutung ist (Miller et al. 2009). Bei Patienten mit kongenitalen Herzvitien zeigten konventionelle CINE-Flussmessungen mit anschließender Phantom-Korrektur Änderungen der pulmonalen Regurgitationsfraktion von bis zu 27 \% (Holland et al. 2010). Basierend auf diesen Ergebnissen wird bei entsprechenden Fragestellung die Korrektur mit Hilfe eines statischen Phantoms empfohlen (Fratz et al. 2013; Kilner et al. 2010). In der klinischen Routine ist der Einsatz einer korrigierenden Phantommessung allerdings aufwendig und wird deshalb nur unregelmäßig durchgeführt. Die neu entwickelten Echtzeit-CMR-Flussmessungen sollten deshalb in Zukunft innerhalb klinischer Studien, vor allem auch im Bereich des pulmonalen Ausflusstraktes, auf Phasen-Offset-Fehler überprüft werden. Die Ergebnisse könnten mit hoher Wahrscheinlichkeit dazu führen, dass eine zeitintensive korrigierende Phantommessung 
bei zukünftiger Verwendung der Echtzeit-CMR-Flussmessungen im klinischen Alltag nicht mehr erforderlich ist.

Der am Phantom festgestellte Phasen-Offset-Fehler wurde in den Ergebnissen der Probandenmessung nicht verrechnet. Eine entsprechende Verrechnung ist nicht durch eine triviale Subtraktion der ermittelten Flussparameter am Phantom von den Werten aus der dazugehörigen Probandenmessung möglich, sondern müsste pixelweise vorgenommen werden. Entsprechende Software-Funktionen sind für das neu entwickelte Echtzeit-Verfahren derzeit noch nicht verfügbar. Neben der Korrektur von Phasen-Offset-Fehlern mit Hilfe eines statischen Phantoms existieren alternative Ansätze zur automatischen Korrektur durch entsprechende Nachverarbeitungssoftware (Lankhaar et al. 2005; Walker et al. 1993). Die dahinterliegenden Algorithmen orientieren sich direkt an den Phasen-Bildern einer In-vivo-Flussmessung bei Probanden bzw. Patienten. Sie versuchen anhand der Geschwindigkeitsdistribution verschiedener Gewebe alle Pixel des stationären Gewebes (z. B. Brustwand, Wirbelkörper) im FOV zu identifizieren, um daraus näherungsweise den Phasen-Offset-Fehler zu bestimmen. Diese Verfahren müssen zukünftig dahingehend evaluiert werden, ob sie eine zeitintensivere Phantomkorrektur ersetzen können.

\section{Bestimmung hämodynamischer Parameter}

Das neu entwickelte Echtzeit-Verfahren zeigte im Vergleich zu den konventionellen MRT-Flussmessungen eine ebenbürtige Erfassung des Flussvolumens. Darüber hinaus konnten höhere maximale Flussgeschwindigkeiten in den Echtzeit-Messungen dargestellt werden.

Die Echtzeit-CMR-Flussmessung ist nicht auf die Interpolation multipler Herzzyklen angewiesen und ermöglicht somit die Aufnahme von Blutflussprofilen sukzessiver Herzzyklen. In dieser Studie wurde pro Echtzeit-Messung jeweils eine Abfolge von 10 Herzschlägen betrachtet. Die herzgesunden Probanden zeigten dabei eine eindeutig periodische Flussdynamik des Flussvolumens und der Maximalgeschwindigkeit. Dabei handelt es sich um ein bekanntes physiologisches Phänomen, das auf die kardiorespiratorische Kopplung zurückzuführen ist (Ruskin et al. 1973). Beobachtungen von Flussvariationen dieser Art wurden bei MRT-Flussmessungen bisher nicht be- 
schrieben. Die ermittelten Werte des Schlagvolumens und der Maximalgeschwindigkeit zeigten im Vergleich ähnliche, aber nicht identische Werte. Die in freier Atmung durchgeführten Echtzeit-CMR-Flussmessungen zeigten im Vergleich zur durchgeatmeten CINE-Flussmessung (CINE-FB) bis zu Maximalgeschwindigkeiten von $120 \mathrm{~cm}$ $\mathrm{s}^{-1}$ vergleichbare Werte. Bei Maximalgeschwindigkeiten über $120 \mathrm{~cm} \mathrm{~s}^{-1}$ ermitteln die Echtzeit-CMR-Flussmessungen höhere Werte. Die CINE-Flussmessungen tendieren durch die Interpolation multipler Herzzyklen und der damit real schlechteren zeitlichen Auflösung bekanntlich dazu, die maximale Flussgeschwindigkeit zu unterschätzen (Lotz et al. 2002). Die in den zeitlich hochauflösenden Echtzeit-CMRFlussmessungen ermittelten höheren Flussgeschwindigkeiten können deshalb durchaus korrekt sein.

Bei Mittelung der Maximalgeschwindigkeiten zeigten die Echtzeit-CMRFlussmessungen höhere Standardabweichungen als die CINE-Flussmessungen. Dies ist am wahrscheinlichsten auf die beobachteten physiologischen Variationen der Maximalgeschwindigkeit zurückzuführen. Der Vergleich der Maximalgeschwindigkeiten zwischen den beiden abgewandelten Varianten der Echtzeit-CMR-Flussmessung (RT-CMR-Int und RT-CMR-Seq) bestätigt durch eine gute Übereinstimmung mit engen Übereinstimmungsgrenzen und hoher Korrelation die Robustheit und Konsistenz der Echtzeit-Flussmessung bei der Bestimmung maximaler Flussgeschwindigkeiten.

Der Vergleich der ermittelten Schlagvolumina zeigte interessanterweise eine gute Übereinstimmung zum einen zwischen RT-CMR-Seq und CINE-FB, zum anderen zwischen RT-CMR-Int und CINE-BH. Die beiden abgewandelten Formen der Echtzeit-Methode zeigten im Vergleich geringe (im Mittel 8,4\%), jedoch augenscheinlich systematische Abweichungen, wobei RT-CMR-Int höhere Werte aufwies. Eine mögliche Erklärung für diese Abweichung wurde in Abbildung 15 gezeigt: RT-CMR-Int zeigte im Vergleich zu RT-CMR-Seq in der Diastole höhere Durchschnittsgeschwindigkeiten und keine negativen Flüsse in der frühen Diastole. Das Schlagvolumen (Flussvolumen pro Herzschlag) berechnet sich aus dem Produkt der über das Gefäßlumen gemittelten Durchschnittsgeschwindigkeit und der Gefäßquerschnittsfläche mit anschließender Integration über die Zeit eines Herzschlages. Der kurze Rückfluss in der Aorta ascendens in früher Diastole stellt eine bekannte physiologische Besonderheit dar und entspricht dem Rückstrom des Blutes in die Koronararterien (Casper et al. 1956; Seed and Wood 1971). Die unterschiedlichen diastolischen Flussverhält- 
nisse können deshalb zur Diskrepanz der netto gemessenen Flussvolumina zwischen den beiden Echtzeit-Varianten geführt haben. Ein separater, quantitativer Vergleich diastolischer Flussvolumina ist in dieser Studie allerdings nicht erfolgt.

Die beiden Varianten der CINE-Flussmessungen zeigten bei Messung des Schlagvolumens ebenfalls systematische Abweichungen (im Mittel 4,5\%), wobei CINE-BH im Vergleich höhere Werte gemessen hat. Eine mögliche Erklärung für die Abweichung wurde in Abbildung 16 gezeigt: CINE-BH zeigte in maximaler Systole höhere Durchschnittsgeschwindigkeiten als CINE-FB. Während CINE-FB in Ruheatmung durchgeführt werden konnte, wurde bei CINE-BH nach forcierter Inspiration und Exspiration die Luft angehalten. Aus diesem Grund können die Abweichungen der gemessenen Flussvolumina auch auf die verschiedenen physiologischen Zustände während der Messung zurückzuführen sein.

\section{Auswertbarkeit der MRT-Flussmessungen}

Die Zuverlässigkeit der voll-automatischen Auswertung der Echtzeit-Daten konnte für die Analyse von Flussvolumina gezeigt werden. Für die Analyse von maximalen Flussgeschwindigkeiten stellte sich die automatische Auswertung mit manueller Nachkorrektur dagegen als erforderlich heraus.

Die Entwicklung einer Echtzeit-Methode zur quantitativen Flussmessung stellt einige Anforderungen an die Auswertungssoftware zur Funktionsanalyse, die bei konventionellen CINE-Phasenkontrast-Flussmessungen nur eine untergeordnete Rolle gespielt haben. Die Echtzeit-CMR-Flussmessung lieferte bei einer Akquisition von 10 Herzzyklen im Vergleich zur CINE-Phasenkontrast-Flussmessung ca. 8 Mal (CINEFB) bzw. $12 \mathrm{Mal}$ (CINE-BH) so viel Datenmaterial. Alle kommerziell erwerbbaren Analysesoftwares erlauben derzeit keine Auswertung von Datenmengen in diesen Dimensionen. Der auf Grundlage der Software QFLow (MEDIS, Medical Imaging Systems BV, Leiden, Niederlande) entwickelte Prototype erlaubt die Auswertung der anfallenden Datenmengen. Es zeigte sich, dass die Auswertung der Echtzeit-Daten für 10 Herzzyklen ca. 6 Mal so lange dauerte wie die Auswertung eines interpolierten Herzzyklus in den CINE-Flussmessungen. Ein einzelner Herzzyklus der EchtzeitAufnahmen konnte damit durchschnittlich schneller analysiert werden als ein interpo- 
lierter Herzzyklus in den CINE-Flussmessungen. Im Vergleich zu den CINEFlussmessungen zeigten die Bilder der Echtzeit-Daten im zeitlichen Verlauf der Diastole sogar eine bessere Abgrenzbarkeit der aortalen Gefäßwand. Die schlechtere Bildqualität der CINE-Flussmessungen ist hierbei wahrscheinlich auf die Interpolation multipler Herzzyklen zurückzuführen, deren Varianz vor allem im zeitlichen Bereich der Diastole stattfindet. Da ein Großteil der manuellen Korrektur gerade im zeitlichen Bereich der Diastole stattfindet, kann die schnellere Auswertung eines einzelnen Herzzyklus in den Echtzeit-CMR-Flussmessungen auf die bessere Bildqualität in der Diastole zurückzuführen sein. Nichts desto trotz sollte nicht die Auswertungszeit eines singulären Herzzyklus zur Beurteilung der Auswertbarkeit der Echtzeit-Daten herangezogen werden, da gerade die Erfassung von Flussprofilen sukzessiver Herzzyklen einen großen Vorteil der Echtzeit-CMR-Flussmessung darstellt.

Die Bestimmung von Maximalgeschwindigkeiten beruht auf der Definition eines einzelnen Pixels und muss daher immer mit Vorsicht durchgeführt werden (Kilner et al. 2007). Es wurde gezeigt, dass die manuelle Konturkorrektur in den Echtzeit-CMRFlussmessungen signifikante Änderungen der Maximalgeschwindigkeit mit sich bringen kann. Der wahrscheinlichste Grund dafür ist eine Tendenz zur Überschätzung der Gefäßkontur des automatischen Segmentationsalgorithmus. Idealerweise sollte die Kontur deckungsgleich zur Intima des zu analysierenden Gefäßes liegen. Ist die Kontur allerdings weiter außen lokalisiert, steigt die Wahrscheinlichkeit maximale Flussgeschwindigkeiten eines Pixels zu definieren, dessen Phase durch Artefakte oder Rauschen entsteht (Chai and Mohiaddin 2005).

Interessanterweise konnte dagegen kein signifikanter Unterschied des Schlagvolumens durch die manuelle Konturkorrektur bei der Analyse der Echtzeit-CMRFlussmessungen gezeigt werden. Als Konsequenz dieser Beobachtungen ergibt sich, dass die zeitaufwändige manuelle Konturkorrektur im Rahmen zukünftiger klinischen Studien, bei alleiniger Bestimmung des Flussvolumens in der Aorta ascendens - abgesehen von groben Konturfehlern - nicht mehr zwingend erforderlich ist. Um dagegen eine zuverlässige Ermittlung von Maximalgeschwindigkeiten zu gewährleisten, sollte weiterhin stets die manuelle Konturkorrektur vorgenommen werden. Mathematische Weiterentwicklungen des automatischen Segmentationsalgorithmus werden angestrebt, um auch die Bestimmung von Maximalgeschwindigkeiten in der Aorta ascendens zukünftig voll-automatisch vornehmen zu können (mündliche Aus- 
kunft der Software-Entwickler der Firma MEDIS, Medical Imaging Systems BV, Leiden, Niederlande).

\section{Vergleich mit der Doppler-Echokardiographie}

Die Maximalgeschwindigkeiten der MRT-Flussmessungen wurden mit Werten aus der Doppler-Echokardiographie als klinischer Goldstandard verglichen. Dieser Vergleich bietet sich an, da die Doppler-echokardiographische Flussmessung ebenfalls ein Echtzeit-Verfahren darstellt. Die Ergebnisse der vorliegenden Studie zeigten im Vergleich keine signifikant unterschiedlichen Ergebnisse der maximalen Flussgeschwindigkeiten zwischen MRT- und Doppler-Flussmessung. Die mittlere Differenz war gering, wohingegen die Streuung der Messwerte sehr hoch war, was sich in weiten Übereinstimmungsgrenzen in der Bland-Altman-Analyse widerspiegelte.

Der Vergleich von Maximalgeschwindigkeiten zwischen konventioneller CINEFlussmessung und Doppler-echokardiographischer Messung ist vielfach in vivo erfolgt (Kilner et al. 1993; Mohiaddin et al. 1997; Zananiri et al. 1993). In Abhängigkeit des Studiendesigns wurden zum Teil gute und zum Teil schlechte Übereinstimmungen und Korrelationen der Maximalgeschwindigkeiten dokumentiert. In-VitroVergleiche an kontinuierlichen und pulsatilen Flussphantomen zeigten dagegen konsequent eine Unterschätzung der Maximalgeschwindigkeit der CINEFlussmessungen im Vergleich zur Doppler-Echokardiographie (Hosten et al. 1998; Siegel et al. 1996). Die Unterschätzung ist dabei am ehesten auf die real schlechtere zeitliche Auflösung und dem bekannten Problem der Phasen-Mittelung in den CINEFlussmessungen zurückzuführen (Siegel et al. 1996).

Die MRT-Untersuchungen und Doppler-Messungen können aufgrund technischer Limitationen nicht simultan durchgeführt werden. Es mussten deshalb Werte verglichen werden, die zu unterschiedlichen Zeitpunkten gemessen wurden. Alle echokardiographischen Messungen wurden in dieser Studie von einem erfahrenen Untersucher durchgeführt. Es handelt sich trotzdem um ein stark untersucherabhängiges Verfahren. Die Doppler-echokardiographische Flussbestimmung unterliegt einer groBen Intra- und Interobserver-Variabilität, die in dieser Studie nicht weiter analysiert wurde (Lui et al. 2005; Tessler et al. 1990). Bei zwei Probanden zeigten die Doppler- 
echokardiographischen Werte im Vergleich zu allen MRT-Flussmessungen eine deutliche Unterschätzung. Maximalgeschwindigkeiten, die bei Dopplerechokardiographischen Flussgeschwindigkeitsmessungen wie in diesem Fall deutlich unter dem erwarteten Wert liegen, sind am wahrscheinlichsten auf die Schwierigkeit zurückzuführen ein Schallfenster mit adäquatem Doppler-Signal zu erhalten (Lui et al. 2005). Eine genaue Doppler-echokardiographische Bestimmung der Maximalgeschwindigkeit ist weiterhin nur möglich, wenn der Schallstrahl genau im Jet des Blutflusses lokalisiert ist. Der Abweichungswinkel zwischen Schallstrahl und Jet ist ausschlaggebend für die Intra- und Interobservervariabilität, die je nach maximaler Flussgeschwindigkeit Abweichungen von bis zu $25 \%$ (Variationskoeffizient) hervorrufen kann (Hadlock und Beach 2009; Hoskins 1996).

Die Robustheit und Konsistenz der MRT-Flussmessungen bei der Bestimmung maximaler Flussgeschwindigkeiten wurde gezeigt. Die Streuung der Werte ist am ehesten auf die Limitationen des Studiendesigns (nicht-simultane Flussmessung) und die hohe Variabilität bei der Durchführung der Doppler-echokardiographischen Flussmessungen zurückzuführen.

\subsection{Anwendung der Echtzeit-CMR-Flussmessung mit dem Valsalva-Manöver als physiologischem Stressor}

Die Ergebnisse der Echtzeit-CMR-Flussmessungen unter dem Valsalva-Manöver haben gezeigt, dass die Vorteile der neu entwickelten Technik (Darstellung von Variationen sukzessiver Herzzyklen, Unabhängigkeit einer Atemkompensation) effektiv genutzt werden konnten, um physiologische Variationen des Blutflusses unter erhöhtem intrathorakalen Druck quantitativ zu ermitteln. Die Ergebnisse der Änderungen des Flussvolumens unter dem Valsalva-Manöver entsprechen den Beobachtungen aus invasiv ermittelten Werten aus dem Herzkatheterlabor (Greenfield et al. 1967).

Die hämodynamischen Schwankungen unter erhöhten intrathorakalen Druck konnten im Rahmen der Studie erstmals mit einer MRT-basierten Echtzeit-Flussmessung in einer größeren Probandengruppe nachgewiesen werden. Frühere Studien konnten die Effekte des Valsalva-Manövers in kleineren Probanden- bzw. Patientengruppen mit zum Teil großen Variationen beobachten (Attubato et al. 1994; Brooker et al. 
1974; Eichenberger et al. 1995). Die dabei beobachteten starken Variationen waren am ehesten auf die unterschiedliche Durchführung des Valsalva-Manövers zwischen den Probanden zurückzuführen. Aus diesem Grund wurde in der vorliegenden Studie besonderer Wert auf eine standardisierte Durchführung gelegt. Der intraorale Druck wurde während den Echtzeit-CMR-Flussmessungen über ein digitales Manometer aufgezeichnet und den Probanden als Biofeedback über eine LCD-Brille präsentiert. Die visuelle Kontrolle führte zur exakten und reproduktiven Durchführung des Valsalva-Manövers über einen fest definierten Zeitraum von 10 Sekunden. Die Möglichkeit der freien Wahl einer Messebene in der MRT ermöglichte eine Schichtposition zu wählen, in der der Blutfluss simultan in der oberen Hohlvene und der aufsteigenden Aorta gemessen wurde. Die gleichzeitige Analyse von Flussvolumen, Gefäßquerschnittsfläche und Herzfrequenz in beiden Gefäßen erlaubte eine detaillierte Darstellung des Zusammenspiels von Vorlast, Nachlast und reflektorischer Herzfrequenzmodulation unter erhöhten intrathorakalen Druck (Looga 2001; Looga 2002; Robertson et al. 1977). Frühere Studien haben versucht die hämodynamischen Effekte des Valsalva-Manövers echokardiographisch zu bestimmen (Gindea et al. 1990; Parisi et al. 1976). Die Doppler-echokardiographische Flussmessung ist auf die Erfassung der Flussgeschwindigkeiten in einem Gefäß beschränkt. Eine gleichzeitige Messung von Flussvolumen und Gefäßquerschnittsfläche ist nicht möglich. Gindea et al. haben versucht die Auswirkung des Valsalva-Manövers in der oberen Hohlvene echokardiographisch darzustellen (Gindea et al. 1990). Die Messung der Gefäßquerschnittsfläche war dabei lediglich bei $39 \%$ der Probanden möglich. Die EchtzeitCMR-Flussmessung bietet im Vergleich dazu ein robusteres und schnelles Verfahren zur Darstellung hämodynamischer Variationen unter erhöhten intrathorakalen Druck. Die Flussmessungen konnte bei allen Probanden erfolgreich durchgeführt und ausgewertet werden. Die adäquate Ableitung eines EKG-Signals unter Anspannung der Atemmuskulatur stellte sich bei einigen Probanden als limitierender Faktor heraus. In diesen Fällen musste die Messung der statistischen Analyse entzogen werden.

Die kardiale Reaktion auf das Valsalva-Manöver wird klinisch verwendet werden, um echokardiographisch den Grad der linksventrikulären diastolischen Dysfunktion zu bestimmen (Weidemann et al. 2013). Auch für die kardiale MRT werden zunehmend Techniken entwickelt, die eine Analyse der diastolischen Relaxation erlauben (Kowallick et al. 2014 a; Kowallick et al. 2014 c; Kowallick et al. 2014 d). Aktuell wird 
das in der vorliegenden Arbeit beschriebene Verfahren bei Patienten mit Herzinsuffizienz mit erhaltener linksventrikulärer Ejektionsfraktion angewendet. Abnorme hämodynamische Reaktionen könnten dabei helfen, den bisher unzureichend geklärten Pathomechanismus dieser Erkrankung genauer zu verstehen. Die Ergebnisse der vorliegenden Arbeit werden in diesem Zusammenhang als hämodynamische Referenzantwort herzgesunder Probanden verwendet. 


\section{Zusammenfassung}

Ziel der vorliegenden Arbeit war die Evaluation eines neu entwickelten EchtzeitVerfahrens zur quantitativen Flussmessung. Die in vitro erhobenen Messungen zeigten weniger Phasen-Offset-Fehler in den Echtzeit-Flussmessungen. Die in vivo ermittelten Ergebnisse bestätigten eine zuverlässige Erfassung des Flussvolumens in der aufsteigenden Aorta. Im Vergleich zu den CINE-Flussmessungen wurden höhere Maximalgeschwindigkeiten gemessen, die aufgrund der real besseren zeitlichen Auflösung durchaus richtig sein können. Die Doppler-Echokardiographie stellte in diesem Zusammenhang keinen zuverlässigen Referenzstandard dar. Eine schnelle, voll-automatische Auswertung der Echtzeit-Daten ist bei alleiniger Bestimmung von Flussvolumina akzeptabel, während bei der Bestimmung maximaler Flussgeschwindigkeiten eine manuelle Konturkorrektur empfohlen wird. Die Echtzeit-Flussmessung erlaubte die Darstellung von Flussprofilen sukzessiver Herzzyklen. Diese Möglichkeit wurde genutzt, um die hämodynamischen Variationen unter einem physiologischen Stressor wie dem Valsalva-Manöver detailliert und zuverlässig darzustellen.

Die Echtzeit-CMR-Flussmessung bietet im Vergleich zur konventionellen CINEFlussmessung überzeugende Vorteile für den klinischen Einsatz:

1. Unempfindlichkeit gegenüber Phasen-Offset-Fehlern: Eine zeitaufwändige Phantomkorrektur dürfte in der klinischen Routine nicht mehr erforderlich sein.

2. Höhere maximale Flussgeschwindigkeiten: eine Evaluation stenosierter Herzklappen oder Gefäße könnte zuverlässiger vorgenommen werden.

3. Keine Notwendigkeit einer Atemkompensation: eine zuverlässige Flussmessung mit deutlicher Reduktion der Untersuchungszeit wird auch bei Patienten möglich, die den Atem konstitutionsbedingt oder aufgrund ihrer Erkrankung nicht über einen längeren Zeitraum anhalten können (z. B. Kinder).

4. Keine Notwendigkeit einer EKG-Synchronisation: Eine zuverlässige Flussbestimmung wird auch bei Patienten mit kardialen Arrhythmien möglich sein.

5. Darstellung von beat-to-beat-Flussvariationen: Abnorme hämodynamische Variationen (z. B. unter dem Valsalva-Manöver) könnten als Biomarker bei unterschiedlichen kardiovaskulären Krankheitsbildern dienen.

Die aufgeführten Vorteile der Echtzeit-Flussmessung müssen zukünftig innerhalb klinischer Studien bestätigt werden. 


\section{Literaturverzeichnis}

Achenbach S, Barkhausen J, Beer M, Beerbaum P, Dill T, Eichhorn J, Fratz S, Gutberlet M, Hoffmann M, Huber A et al. (2012): [Consensus recommendations of the German Radiology Society (DRG), the German Cardiac Society (DGK) and the German Society for Pediatric Cardiology (DGPK) on the use of cardiac imaging with computed tomography and magnetic resonance imaging]. Rofo 184, 345-368

Attubato MJ, Katz ES, Feit F, Bernstein N, Schwartzman D, Kronzon I (1994): Venous changes occurring during the Valsalva maneuver: evaluation by intravascular ultrasound. Am J Cardiol $\underline{74}$, 408-410

Beerbaum P, Parish V, Bell A, Gieseke J, Korperich H, Sarikouch S (2008): Atypical atrial septal defects in children: noninvasive evaluation by cardiac MRI. Pediatr Radiol $\underline{38}, 1188-1194$

Bland JM, Altman DG (1999): Measuring agreement in method comparison studies. Stat Methods Med Res $\underline{8}, 135-160$

Blechinger JC, Madsen EL, Frank GR (1988): Tissue-mimicking gelatin-agar gels for use in magnetic resonance imaging phantoms. Med Phys $\underline{15}, 629-636$

Brooker JZ, Alderman EL, Harrison DC (1974): Alterations in left ventricular volumes induced by Valsalva manoeuvre. Br Heart J $\underline{36}, 713-718$

Caprihan A, Altobelli S, Benitez-Read E (1990): Flow-Velocity Imaging from linear regression of phase images with techniques for reducing eddy-current effects. $J$ Magn Reson Imaging 90, 71-89

Casper AG, Fry DL, Mallos AJ (1956): A catheter tip method for measurement of the instantaneous aortic blood velocity. Circ Res $\underline{4}$, 627-632

Cawley PJ, Maki JH, Otto CM (2009): Cardiovascular magnetic resonance imaging for valvular heart disease: technique and validation. Circulation $\underline{119}$, 468-478 
Chai P, Mohiaddin R (2005): How we perform cardiovascular magnetic resonance flow assessment using phase-contrast velocity mapping. J Cardiovasc Magn Reson 7, $705-716$

Chernobelsky A, Shubayev O, Comeau CR, Wolff SD (2007): Baseline correction of phase contrast images improves quantification of blood flow in the great vessels. $J$ Cardiovasc Magn Reson $\underline{9}$, 681-685

Debatin JF, Davis CP, Felblinger J, McKinnon GC (1995 a): Evaluation of ultrafast phase-contrast imaging in the thoracic aorta. Magma $\underline{3}, 59-66$

Debatin JF, Leung DA, Wildermuth S, Botnar R, Felblinger J, McKinnon GC (1995 b): Flow quantitation with echo-planar phase-contrast velocity mapping: in vitro and in vivo evaluation. J Magn Reson Imaging $\underline{5}, 656-662$

Eichenberger AC, Schwitter J, McKinnon GC, Debatin JF, von Schulthess GK (1995): Phase-contrast echo-planar MR imaging: real-time quantification of flow and velocity patterns in the thoracic vessels induced by Valsalva's maneuver. J Magn Reson Imaging $\underline{5}, 648-655$

Felker GM, Cuculich PS, Gheorghiade M (2006): The Valsalva maneuver: a bedside "biomarker" for heart failure. Am J Med 119, 117-122

Ferreira PF, Gatehouse PD, Mohiaddin RH, Firmin DN (2013): Cardiovascular magnetic resonance artefacts. J Cardiovasc Magn Reson 15, 41

Firmin DN, Nayler GL, Kilner PJ, Longmore DB (1990): The application of phase shifts in NMR for flow measurement. Magn Reson Med 14, 230-241

Fratz S, Chung T, Greil GF, Samyn MM, Taylor AM, Valsangiacomo Buechel ER, Yoo SJ, Powell AJ (2013): Guidelines and protocols for cardiovascular magnetic resonance in children and adults with congenital heart disease: SCMR expert consensus group on congenital heart disease. J Cardiovasc Magn Reson 15, 51 
Gatehouse PD, Rolf MP, Graves MJ, Hofman MB, Totman J, Werner B, Quest RA, Liu Y, von Spiczak J, Dieringer M et al. (2010): Flow measurement by cardiovascular magnetic resonance: a multi-centre multi-vendor study of background phase offset errors that can compromise the accuracy of derived regurgitant or shunt flow measurements. J Cardiovasc Magn Reson 12, 5

Gatehouse PD, Rolf MP, Bloch KM, Graves MJ, Kilner PJ, Firmin DN, Hofman MB (2012): A multi-center inter-manufacturer study of the temporal stability of phasecontrast velocity mapping background offset errors. J Cardiovasc Magn Reson 14, 72

Gindea AJ, Slater J, Kronzon I (1990): Doppler echocardiographic flow velocity measurements in the superior vena cava during the Valsalva maneuver in normal subjects. Am J Cardiol $\underline{65}$, 1387-1391

Greenfield JC, Jr., Cox RL, Hernandez RR, Thomas C, Schoonmaker FW (1967): Pressure-flow studies in man during the Valsalva maneuver with observations on the mechanical properties of the ascending aorta. Circulation $\underline{35}, 653-661$

Guilfoyle DN, Gibbs P, Ordidge RJ, Mansfield P (1991): Real-time flow measurements using echo-planar imaging. Magn Reson Med 18, 1-8

Hadlock J, Beach KW (2009): Velocity variability in ultrasonic Doppler examinations. Ultrasound Med Biol $\underline{35}$, 949-954

Hahn EL (1960): Detection of Sea-Water Motion by Nuclear Precession. J Geophys Res $\underline{65}, 776-777$

Hansen MS, Sorensen TS, Arai AE, Kellman P (2012): Retrospective reconstruction of high temporal resolution cine images from real-time MRI using iterative motion correction. Magn Reson Med 68, 741-750

Holland BJ, Printz BF, Lai WW (2010): Baseline correction of phase-contrast images in congenital cardiovascular magnetic resonance. J Cardiovasc Magn Reson 12, 11 
Hoskins PR (1996): Accuracy of maximum velocity estimates made using Doppler ultrasound systems. Br J Radiol $\underline{69}, 172-177$

Hosten N, Gutberlet M, Kuhne T, Oellinger H, Vogel M, Bockel T, Bock J, Frank J (1998): [Cardiac MR flowmetry: experimental validation and results in patients with operated heart defects]. Rofo $\underline{168}, 480-487$

Joseph AA: Real-time MRI of Moving Spins Using Undersampled Radial FLASH. Phys. Diss. Würzburg 2013

Joseph A, Kowallick JT, Merboldt KD, Voit D, Schaetz S, Zhang S, Sohns JM, Lotz J, Frahm J (2014): Real-time flow MRI of the aorta at a resolution of $40 \mathrm{msec}$. J Magn Reson Imaging 느, 206-213

Joseph AA, Merboldt KD, Voit D, Zhang S, Uecker M, Lotz J, Frahm J (2012): Realtime phase-contrast MRI of cardiovascular blood flow using undersampled radial fast low-angle shot and nonlinear inverse reconstruction. NMR Biomed 25, 917-924

Keenan NG, Pennell DJ (2007): CMR of ventricular function. Echocardiography $\underline{24}$, 185-193

Kilner PJ, Manzara CC, Mohiaddin RH, Pennell DJ, Sutton MG, Firmin DN, Underwood SR, Longmore DB (1993): Magnetic resonance jet velocity mapping in mitral and aortic valve stenosis. Circulation $\underline{87}, 1239-1248$

Kilner PJ, Gatehouse PD, Firmin DN (2007): Flow measurement by magnetic resonance: a unique asset worth optimising. J Cardiovasc Magn Reson $\underline{9}$, 723-728

Kilner PJ, Geva T, Kaemmerer H, Trindade PT, Schwitter J, Webb GD (2010): Recommendations for cardiovascular magnetic resonance in adults with congenital heart disease from the respective working groups of the European Society of Cardiology. Eur Heart J $\underline{31}, 794-805$ 
Kimme-Smith C, Hussain R, Duerinckx A, Tessler F, Grant E (1990): Assurance of consistent peak-velocity measurements with a variety of duplex Doppler instruments. Radiology 177, 265-272

Korperich H, Gieseke J, Barth P, Hoogeveen R, Esdorn H, Peterschroder A, Meyer $H$, Beerbaum P (2004): Flow volume and shunt quantification in pediatric congenital heart disease by real-time magnetic resonance velocity mapping: a validation study. Circulation 109, 1987-1993

Kowallick JT, Edelmann F, Lotz J, Lamata P, Schuster A (2014 a): Imaging Diastolic Dysfunction with Cardiovascular Magnetic Resonance. Journal of Cardiol Ther $\underline{1}, 58-$ 64

Kowallick JT, Joseph AA, Unterberg-Buchwald C, Fasshauer M, van Wijk K, Merboldt KD, Voit D, Frahm J, Lotz J, Sohns JM (2014 b): Real-time phase-contrast flow MRI of the ascending aorta and superior vena cava as a function of intrathoracic pressure (Valsalva manoeuvre). Br J Radiol 구, 20140401

Kowallick JT, Kutty S, Edelmann F, Chiribiri A, Villa A, Steinmetz M, Sohns JM, Staab W, Bettencourt N, Unterberg-Buchwald C et al. (2014 c): Quantification of left atrial strain and strain rate using Cardiovascular Magnetic Resonance myocardial feature tracking: a feasibility study. J Cardiovasc Magn Reson 16, 60

Kowallick JT, Lamata P, Hussain ST, Kutty S, Steinmetz M, Sohns JM, Fasshauer M, Staab W, Unterberg-Buchwald C, Bigalke B et al. (2014 d): Quantification of left ventricular torsion and diastolic recoil using cardiovascular magnetic resonance myocardial feature tracking. PLoS One $\underline{9}$, e109164

Kozerke S, Schwitter J, Pedersen EM, Boesiger P (2001): Aortic and mitral regurgitation: quantification using moving slice velocity mapping. J Magn Reson Imaging 14, 106-112 
Lalande S, Luoma CE, Miller AD, Johnson BD (2012): Effect of changes in intrathoracic pressure on cardiac function at rest and during moderate exercise in health and heart failure. Exp Physiol $\underline{97}$, 248-256

Lankhaar JW, Hofman MB, Marcus JT, Zwanenburg JJ, Faes TJ, Vonk-Noordegraaf A (2005): Correction of phase offset errors in main pulmonary artery flow quantification. J Magn Reson Imaging 22, 73-79

Lardo AC, Abraham TP, Kass DA (2005): Magnetic resonance imaging assessment of ventricular dyssynchrony: current and emerging concepts. J Am Coll Cardiol 46, 2223-2228

Lauterbur PC (1973): Image formation by induced local interactions. Examples employing nuclear magnetic resonance. Nature $\underline{242}, 190-191$

Looga R (2001): The bradycardic response to the Valsalva manoeuvre in normal man. Respir Physiol 124, 205-215

Looga $R$ (2002): Reproducibility of the heart rate response to low-strain Valsalva manoeuvre in healthy subjects. Respir Physiol Neurobiol 133, 251-258

Lotz J, Meier C, Leppert A, Galanski M (2002): Cardiovascular flow measurement with phase-contrast MR imaging: basic facts and implementation. Radiographics $\underline{22}$, 651-671

Lui EY, Steinman AH, Cobbold RS, Johnston KW (2005): Human factors as a source of error in peak Doppler velocity measurement. J Vasc Surg $\underline{42}$, 972-979

Macgowan CK, Kellenberger CJ, Detsky JS, Roman K, Yoo SJ (2005): Real-time Fourier velocity encoding: an in vivo evaluation. J Magn Reson Imaging 21, 297-304

Makowski MR, Wiethoff AJ, Uribe S, Parish V, Botnar RM, Bell A, Kiesewetter C, Beerbaum P, Jansen CH, Razavi R et al. (2011): Congenital heart disease: 
cardiovascular MR imaging by using an intravascular blood pool contrast agent. Radiology 260, 680-688

Mansfield P, Maudsley AA (1977): Medical imaging by NMR. Br J Radiol 므, 188-194

McVeigh ER, Prinzen FW, Wyman BT, Tsitlik JE, Halperin HR, Hunter WC (1998): Imaging asynchronous mechanical activation of the paced heart with tagged MRI. Magn Reson Med 39, 507-513

Miller TA, Landes AB, Moran AM (2009): Improved accuracy in flow mapping of congenital heart disease using stationary phantom technique. J Cardiovasc Magn Reson $\underline{11}, 52$

Mohiaddin RH, Gatehouse PD, Henien M, Firmin DN (1997): Cine MR Fourier velocimetry of blood flow through cardiac valves: comparison with Doppler echocardiography. J Magn Reson Imaging 프, 657-663

Moran PR, Moran RA, Karstaedt N (1985): Verification and evaluation of internal flow and motion. True magnetic resonance imaging by the phase gradient modulation method. Radiology 154, 433-441

Niebergall A, Zhang S, Kunay E, Keydana G, Job M, Uecker M, Frahm J (2013): Real-time MRI of speaking at a resolution of $33 \mathrm{~ms}$ : undersampled radial FLASH with nonlinear inverse reconstruction. Magn Reson Med 69, 477-485

Niendorf T, Sodickson D (2006): [Acceleration of cardiovascular MRI using parallel imaging: basic principles, practical considerations, clinical applications and future directions]. Rofo $\underline{178}, 15-30$

O'Donnell M (1985): NMR blood flow imaging using multiecho, phase contrast sequences. Med Phys $\underline{12}$, 59-64 
Parisi AF, Harrington JJ, Askenazi J, Pratt RC, Mclntyre KM (1976): Echocardiographic evaluation of the Valsalva Maneuver in healthy subjects and patients with and without heart failure. Circulation $\underline{54}$, 921-927

Pelc NJ, Bernstein MA, Shimakawa A, Glover GH (1991): Encoding strategies for three-direction phase-contrast MR imaging of flow. J Magn Reson Imaging 1 , 405413

Petersson S, Dyverfeldt P, Gardhagen R, Karlsson M, Ebbers T (2010): Simulation of phase contrast MRI of turbulent flow. Magn Reson Med 64, 1039-1046

Pruessmann KP, Weiger M, Scheidegger MB, Boesiger P (1999): SENSE: sensitivity encoding for fast MRI. Magn Reson Med 42, 952-962

Robertson D, Stevens RM, Friesinger GC, Oates JA (1977): The effect of the Valsalva maneuver on echocardiographic dimensions in man. Circulation $\underline{55}, 596-$ 562

Ruskin J, Bache RJ, Rembert JC, Greenfield JC, Jr. (1973): Pressure-flow studies in man: effect of respiration on left ventricular stroke volume. Circulation $\underline{48}, 79-85$

Seed WA, Wood NB (1971): Velocity patterns in the aorta. Cardiovasc Res $\underline{5}, 319$ 330

Siegel JM, Jr., Oshinski JN, Pettigrew RI, Ku DN (1996): The accuracy of magnetic resonance phase velocity measurements in stenotic flow. J Biomech $\underline{29}, 1665-1672$

Spritzer CE, Pelc NJ, Lee JN, Evans AJ, Sostman HD, Riederer SJ (1990): Rapid MR imaging of blood flow with a phase-sensitive, limited-flip-angle, gradient recalled pulse sequence: preliminary experience. Radiology $\underline{176}$, 255-262

Steeden JA, Atkinson D, Taylor AM, Muthurangu V (2010): Split-acquisition real-time CINE phase-contrast MR flow measurements. Magn Reson Med 64, 1664-1670 
Tang C, Blatter DD, Parker DL (1993): Accuracy of phase-contrast flow measurements in the presence of partial-volume effects. J Magn Reson Imaging $\underline{3}$, 377-385

Tessler FN, Kimme-Smith C, Sutherland ML, Schiller VL, Perrella RR, Grant EG (1990): Inter- and intra-observer variability of Doppler peak velocity measurements: an in-vitro study. Ultrasound Med Biol $\underline{16}, 653-657$

Thunberg P, Emilsson K, Rask P, Kahari A (2012): Flow and peak velocity measurements in patients with aortic valve stenosis using phase contrast MR accelerated with k-t BLAST. Eur J Radiol 81, 2203-2207

Uecker M, Hohage T, Block KT, Frahm J (2008): Image reconstruction by regularized nonlinear inversion--joint estimation of coil sensitivities and image content. Magn Reson Med 므, 674-682

Uecker M, Zhang S, Frahm J (2010 a): Nonlinear inverse reconstruction for real-time MRI of the human heart using undersampled radial FLASH. Magn Reson Med $\underline{63}$, 1456-1462

Uecker M, Zhang S, Voit D, Karaus A, Merboldt KD, Frahm J (2010 b): Real-time $\mathrm{MRI}$ at a resolution of $20 \mathrm{~ms}$. NMR Biomed $\underline{23}, 986-994$

Walker PG, Cranney GB, Scheidegger MB, Waseleski G, Pohost GM, Yoganathan AP (1993): Semiautomated method for noise reduction and background phase error correction in MR phase velocity data. J Magn Reson Imaging $\underline{3}, 521-530$

Warnes CA, Williams RG, Bashore TM, Child JS, Connolly HM, Dearani JA, Del Nido P, Fasules JW, Graham TP, Jr., Hijazi ZM et al. (2008): ACC/AHA 2008 Guidelines for the Management of Adults with Congenital Heart Disease: Executive Summary: a report of the American College of Cardiology/American Heart Association Task Force on Practice Guidelines (writing committee to develop guidelines for the management of adults with congenital heart disease). Circulation $\underline{118}, 2395-2451$ 
Weidemann F, Niemann M, Herrmann S, Ertl G, Stork S (2013): [Assessment of diastolic heart failure. Current role of echocardiography]. Herz $\underline{38}, 18-25$

Zananiri FV, Jackson PC, Halliwell M, Harris RA, Hayward JK, Davies ER, Wells PN (1993): A comparative study of velocity measurements in major blood vessels using magnetic resonance imaging and Doppler ultrasound. $\mathrm{Br} \mathrm{J}$ Radiol $\underline{66}, 1128-1133$

Zhang S, Block KT, Frahm J (2010 a): Magnetic resonance imaging in real time: advances using radial FLASH. J Magn Reson Imaging $\underline{31}$, 101-109

Zhang S, Uecker M, Voit D, Merboldt KD, Frahm J (2010 b): Real-time cardiovascular magnetic resonance at high temporal resolution: radial FLASH with nonlinear inverse reconstruction. J Cardiovasc Magn Reson 12, 39

Zhang S, Olthoff A, Frahm J (2012): Real-time magnetic resonance imaging of normal swallowing. J Magn Reson Imaging $\underline{35}$, 1372-1379 


\section{Publikationsliste}

\section{Originalarbeiten}

Kowallick JT, Joseph AA, Unterberg-Buchwald C, Fasshauer M, van Wijk K, Merboldt KD, Voit D, Frahm J, Lotz J, Sohns JM (2014): Real-time phase-contrast flow MRI of the ascending aorta and superior vena cava as a function of intrathoracic pressure (Valsalva manoeuvre). Br J Radiol 7, 20140401

Joseph A, Kowallick JT, Merboldt KD, Voit D, Schaetz S, Zhang S, Sohns JM, Lotz J, Frahm J (2014): Real-time flow MRI of the aorta at a resolution of $40 \mathrm{msec}$. J Magn Reson Imaging 느, 206-213

\section{Kongressbeiträge}

Kowallick JT, Sohns JM, Unterberg-Buchwald C, Schulte C, Staab W, Merboldt KD, Voit D, Zhang S, Joseph AA, Frahm J, Lotz J (2013): Hochauflösende EchtzeitCardio-MRT Flussmessung im Vergleich zur Cine Phasenkontrastflussmessung. Vortrag im Young-Investigator-Award Finale, DRG Röntgenkongress 2013, Hamburg. Fortschr Röntgenstr 185 (S 1), VO105_I_4

Kowallick JT, Sohns JM, Joseph AA, Merboldt KD, Voit D, Zhang S, Schulte C, Staab W, Unterberg-Buchwald C, Frahm J, Lotz J (2013): Echtzeit-Cardio-MRTPhasenkontrast-Flussmessung mit dem Valsalva-Manöver als physiologischer Stressor. Initiale klinische Ergebnisse. 79. Jahrestagung der DGK 2013, Mannheim. Clin Res Cardiol 102 (Suppl 1), P758

Kowallick JT, Sohns JM, Unterberg-Buchwald C, Joseph AA, Merboldt KD, Voit D, Zhang S, Schulte S, Staab W, Frahm J, Lotz J (2013): Echtzeit-Cardio-MRTPhasenkontrast-Flussmessung mit dem Valsalva-Manöver als Stresstest: Initiale klinische Ergebnisse. Kardiodiagnostiktage 2013, Leipzig. P 42 
Kowallick JT, Sohns JM, Joseph AA, Merboldt KD, Voit D, Zhang S, Schulte C, Staab W, Unterberg-Buchwald C, Frahm J, Lotz J (2013): Hochauflösende EchtzeitCardio-MRT Flussmessung im Vergleich zur Cine Phasenkontrastflussmessung. Kardiodiagnostiktage 2013, Leipzig. P44

Sohns JM, Kowallick JT, Unterberg-Buchwald C, Staab W, Joseph AA, Merboldt KD, Uecker M, Voit D, Zhang S, Frahm J, Lotz J (2013): Echtzeit-Cardio-MRTPhasenkontrastflussmessung mit dem Valsalva- und Müllermaneuver als Stresstest. Vortrag im Young-Investigator-Award Finale, DRG Röntgenkongress 2013, Hamburg. Fortschr Röntgenstr 185 (S 1), VO105_I_5

Lotz J, Sohns JM, Steinmetz M, Kowallick JT, Schulte C, Staab W, Joseph AA, Merboldt KD, Voit D, Zhang S, Uecker M, Unterberg-Buchwald C, Hasenfuss G, Frahm J (2013): High resolution real-time CMR of function and flow: initial clinical results. 16th Annual SCMR Scientific Sessions 2013, San Francisco. J Cardiovasc Magn Reson 15 (Suppl 1), E99

Sohns JM, Unterberg-Buchwald C, Kowallick JT, Steinmetz M, Schulte C, Staab W, Joseph AA, Merboldt KD, Voit D, Zhang S, Uecker M, Frahm J, Lotz J (2013): Realtime cardiac phase contrast MRI blood flow including Valsalva and Mueller maneuver. Initial experiences. 16th Annual SCMR Scientific Sessions 2013, San Francisco. J Cardiovasc Magn Reson 15 (Suppl 1), E17

Joseph AA, Sohns JM, Kowallick JT, Merboldt KD, Lotz J, Frahm J (2013): Realtime cardiovascular phase-contrast flow MRI during Valsalva Maneuver. Annual Meeting ISMRM 2013, Salt Lake City. Proc. Intl. Soc. Mag. Reson. Med. 21, 4441

Sohns JM, Fasshauer M, Kowallick JT, Schuster A, Staab W, Joseph AA, Zhang S, Voit D, Schaetz S, Merboldt KD, Steinmetz M, Unterberg-Buchwald C, Frahm J, Lotz $J$ (2014): Simultaneous flow dynamics in small and great thoracic vessels during physiological stress tests and normal breathing using real-time cardiac magneticresonance. 17th Annual SCMR Scientific Sessions 2014, New Orleans. J Cardiovasc Magn Reson 16 (Suppl 1), P9 


\section{Danksagung}

Herrn Prof. Dr. Joachim Lotz möchte ich ganz herzlich für die Überlassung des Themas, die Betreuung der Arbeit und die stetige Vermittlung von Motivation und Begeisterung an dem Projekt danken.

Herrn Prof. Dr. Jens Frahm und Herrn Dr. Arun Antony Joseph möchte ich ganz herzlich für die freundliche Unterstützung von physikalischer Seite danken.

Herrn Dr. Jan Martin Sohns danke ich für die Betreuung und freundschaftliche Zusammenarbeit.

Frau Prof. Dr. Christina Unterberg-Buchwald danke ich für die Durchführung der echokardiographischen Untersuchungen.

Frau Tanja Otto und Frau Anne Weber für die Einarbeitung und die Unterstützung bei der Studienorganisation.

Der Medizinischen Fakultät der Georg-August-Universität Göttingen für die Unterstützung im Rahmen eines Promotionsstipendiums.

Allen Probanden, die sich für die Studien zur Verfügung gestellt haben. 